\title{
Blanchfield and Seifert algebra in high-dimensional boundary link theory I: Algebraic $K$-theory
}

\author{
ANDREW RANICKI \\ DESMOND SHEIHAM
}

\begin{abstract}
The classification of high-dimensional $\mu$-component boundary links motivates decomposition theorems for the algebraic $K$-groups of the group ring $A\left[F_{\mu}\right]$ and the noncommutative Cohn localization $\Sigma^{-1} A\left[F_{\mu}\right]$, for any $\mu \geqslant 1$ and an arbitrary ring $A$, with $F_{\mu}$ the free group on $\mu$ generators and $\Sigma$ the set of matrices over $A\left[F_{\mu}\right]$ which become invertible over $A$ under the augmentation $A\left[F_{\mu}\right] \rightarrow A$. Blanchfield $A\left[F_{\mu}\right]-$ modules and Seifert $A$-modules are abstract algebraic analogues of the exteriors and Seifert surfaces of boundary links. Algebraic transversality for $A\left[F_{\mu}\right]$-module chain complexes is used to establish a long exact sequence relating the algebraic $K-$ groups of the Blanchfield and Seifert modules, and to obtain the decompositions of $K_{*}\left(A\left[F_{\mu}\right]\right)$ and $K_{*}\left(\Sigma^{-1} A\left[F_{\mu}\right]\right)$ subject to a stable flatness condition on $\Sigma^{-1} A\left[F_{\mu}\right]$ for the higher $K$-groups.
\end{abstract}

19D50, 57Q45; 20E05

Desmond Sheiham died 25 March 2005.

This paper is dedicated to the memory of Paul Cohn and Jerry Levine.

\section{Introduction}

For any integer $\mu \geqslant 1$ let $F_{\mu}$ be the free group on $\mu$ generators $z_{1}, z_{2}, \ldots, z_{\mu}$. The classification theory of high-dimensional $\mu$-component boundary links involves 'Seifert $\mathbb{Z}$-modules' and 'Blanchfield $\mathbb{Z}\left[F_{\mu}\right]$-modules', corresponding to the algebraic invariants obtained from $\mu$-component Seifert surfaces and the boundary link exterior. This paper concerns the algebraic relationship between f.g. projective Seifert $A$-modules and h.d. 1 Blanchfield $A\left[F_{\mu}\right]$-modules for any ring $A$, extending the work of Sheiham [41]. Part I deals with the algebraic $K$-theory of the Seifert and Blanchfield modules. Part II will deal with the algebraic $L$-theory of the Seifert and Blanchfield forms, such as arises in the computation of the cobordism groups of boundary links. The algebraic $K$ - and $L$-theory in the knot case $\mu=1$ have already been done by Ranicki [34]. 


\section{Combinatorial transversality}

Section 1 develops a combinatorial construction of fundamental domains for $F_{\mu}$-covers of $C W$ complexes which will serve as a role model for the algebraic transversality of $A\left[F_{\mu}\right]$-module chain complexes in the subsequent sections. The $F_{\mu}$-covers $p: \widetilde{W} \rightarrow W$ of a space $W$ are classified by the homotopy classes of maps

$$
c: W \longrightarrow B F_{\mu}=\bigvee_{\mu} S^{1}
$$

with $\widetilde{W}=c^{*} E F_{\mu}$ the pullback to $W$ of the universal cover $E F_{\mu}$ of $B F_{\mu}$. Let $0 \in B F_{\mu}$ be the point at which the circles $S^{1}$ are joined, and choose points $1,2, \ldots, \mu \in$ $B F_{\mu} \backslash\{0\}$, one in each circle. If $W$ is a compact manifold then $c$ is homotopic to a map which is transverse regular at $\{1,2, \ldots, \mu\} \subset B F_{\mu}$, so that

$$
V=c^{-1}(\{1,2, \ldots, \mu\})=V_{1} \sqcup V_{2} \sqcup \ldots \sqcup V_{\mu} \subset W
$$

is a disjoint union of $\mu$ codimension-1 submanifolds $V_{i}=c^{-1}(\{i\}) \subset W$ (which may be empty) and cutting $W$ at $V$ there is obtained a fundamental domain $U \subset \widetilde{W}$, a compact manifold with boundary

$$
\partial U=\bigsqcup_{i=1}^{\mu}\left(V_{i} \sqcup z_{i} V_{i}\right) .
$$

If $W$ is connected and $c_{*}: \pi_{1}(W) \rightarrow F_{\mu}$ is surjective then $U$ is connected and $V_{1}, V_{2}, \ldots, V_{\mu}$ are non-empty, and may be chosen to be connected. In the combinatorial version of transversality it is only required that $W$ be a finite $C W$ complex, and $W$ may be replaced by a simple homotopy equivalent finite $C W$ complex also denoted by $W$, with disjoint subcomplexes $V_{1}, V_{2}, \ldots, V_{\mu} \subset W$ and a fundamental domain $U \subset \widetilde{W}$ which is a finite subcomplex with a subcomplex

$$
\partial U=\bigsqcup_{i=1}^{\mu}\left(V_{i} \sqcup z_{i} V_{i}\right) \subset U, \quad V_{i}=U \cap z_{i}^{-1} U
$$

such that

$$
\bigcup_{g \in F_{\mu}} g U=\widetilde{W}, g U \cap h U=\varnothing \text { unless } g^{-1} h \in\left\{1, z_{1}, z_{1}^{-1}, \ldots, z_{\mu}, z_{\mu}^{-1}\right\} .
$$

Ranicki [35] developed combinatorial transversality at $Y \subset X$ for maps of finite $C W$ complexes

$$
W \rightarrow X=X_{1} \cup_{Y} X_{2}
$$


with $X, X_{1}, X_{2}, Y$ connected and $\pi_{1}(Y) \rightarrow \pi_{1}\left(X_{1}\right), \pi_{1}(Y) \rightarrow \pi_{1}\left(X_{2}\right)$ injective. The essential difference from [35] is that we are here using the Cayley tree $E F_{\mu}=G_{\mu}$ of $F_{\mu}$ rather than the Bass-Serre tree of the amalgamated free product given by the Seifert-van Kampen Theorem

$$
\pi_{1}(X)=\pi_{1}\left(X_{1}\right) *_{\pi_{1}(Y)} \pi_{1}\left(X_{2}\right)
$$

for bookkeeping purposes. We show that $W$ can be replaced by a simple homotopy equivalent finite $C W$ complex $W$ with disjoint subcomplexes $V_{1}, V_{2}, \ldots, V_{\mu} \subset W$, such that the $F_{\mu}$-cover $\widetilde{W}$ can be constructed from a fundamental domain finite subcomplex $U \subset \widetilde{W}$ obtained by cutting $W$ at $V=V_{1} \sqcup V_{2} \sqcup \ldots \sqcup V_{\mu} \subset W$.

\section{Algebraic transversality}

Let $A$ be an associative ring with 1 . All $A$-modules will be understood to be left $A$-modules, unless a right $A$-module structure is specified.

Section 2 develops an 'algebraic transversality' technique for cutting $A\left[F_{\mu}\right]$-modules along $A$-modules, which mimics the geometric transversality method of Section 1 . In Section 2 we shall prove:

Theorem A Every $A\left[F_{\mu}\right]$-module chain complex $E$ admits a 'Mayer-Vietoris presentation'

$$
0 \longrightarrow \bigoplus_{i=1}^{\mu} C^{(i)}\left[F_{\mu}\right] \stackrel{f}{\longrightarrow} D\left[F_{\mu}\right] \longrightarrow E \longrightarrow 0
$$

with $C^{(i)}, D A$-module chain complexes, and $f=\left(f_{1}^{+} z_{1}-f_{1}^{-} \ldots f_{\mu}^{+} z_{\mu}-f_{\mu}^{-}\right)$ defined using $A$-module chain maps $f_{i}^{+}, f_{i}^{-}: C^{(i)} \rightarrow D$. If $E$ is a f.g. free $A\left[F_{\mu}\right]-$ module chain complex then $C^{(i)}, D$ can be chosen to be f.g. free $A$-module chain complexes, with $D \subset E$ and $f_{i}^{+}, f_{i}^{-}: C^{(i)}=D \cap z_{i}^{-1} D \rightarrow D$ given by $f_{i}^{+}(x)=x$, $f_{i}^{-}(x)=z_{i} x$.

Remark For $\mu=1$ Theorem A was first proved by Waldhausen [47], being the chain complex version of the Higman linearization trick for matrices with entries in the Laurent polynomial extension $A\left[F_{1}\right]=A\left[z, z^{-1}\right]$. The algebraic transversality theory of [47] applies to chain complexes over the group rings $A\left[G_{1} *_{H} G_{2}\right]$ of injective amalgamated free products $G_{1} *_{H} G_{2}$, using the Bass-Serre theory of groups acting on trees. In principle, Theorem A for $\mu \geqslant 2$ could be proved by applying [47] to the successive free products in

$$
F_{\mu}=F_{1} * F_{\mu-1}=F_{1} *\left(F_{1} * F_{\mu-2}\right)=\cdots=F_{1} *\left(F_{1} *\left(F_{1} * \cdots *\left(F_{1}\right)\right)\right)
$$


but this would be quite awkward in practice. In view of both the geometric motivation and the algebraic applications it is better to prove Theorem A (as will be done in Section 2) using the Cayley tree of $F_{\mu}$ with respect to the generator set $\left\{z_{1}, z_{2}, \ldots, z_{\mu}\right\}$.

\section{Boundary links}

A $\mu$-component link is a (locally flat, oriented) embedding

$$
\ell: \bigsqcup_{\mu} S^{n} \subset S^{n+2} \text {. }
$$

Every link admits a Seifert surface $V^{n+1} \subset S^{n+2}$, a codimension-1 submanifold with boundary

$$
\partial V=\ell\left(\bigsqcup_{\mu} S^{n}\right) \subset S^{n+2}
$$

By definition, $\ell$ is a $\mu$-component boundary link if there exists a $\mu$-component Seifert surface

$$
V^{n+1}=V_{1} \sqcup V_{2} \sqcup \ldots \sqcup V_{\mu} \subset S^{n+2} .
$$

The exterior of a link $\ell$ is the $(n+2)$-dimensional manifold with boundary

$$
\left(W^{n+2}, \partial W\right)=\left(\operatorname{cl}\left(S^{n+2}-\left(\ell\left(\bigsqcup_{\mu} S^{n}\right) \times D^{2}\right)\right), \ell\left(\bigsqcup_{\mu} S^{n}\right) \times S^{1}\right) .
$$

In particular, a knot $S^{n} \subset S^{n+2}$ is a 1-component boundary link.

The trivial $\mu$-component boundary link

$$
\ell_{0}: \bigsqcup_{\mu} S^{n} \subset S^{n+2}
$$

is defined by the connected sum of $\mu$ copies of the trivial knot

$$
S^{n} \subset\left(S^{n} \times D^{2}\right) \cup\left(D^{n+1} \times S^{1}\right)=S^{n+2},
$$

so that

$$
\ell_{0}: \bigsqcup_{\mu} S^{n} \subset \underset{\mu}{\#} S^{n+2}=S^{n+2}=\left(\bigsqcup_{\mu} S^{n} \times D^{2}\right) \cup W_{0}
$$

has Seifert surface and exterior

$$
V_{0}=\bigsqcup_{\mu} D^{n+1}, W_{0}=\underset{\mu}{\#}\left(D^{n+1} \times S^{1}\right) \subset S^{n+2} .
$$

The exterior $W_{0}$ has the homotopy type of $\bigvee_{\mu} S^{1} \vee \bigvee_{\mu-1} S^{n+1}$, with $\pi_{1}\left(W_{0}\right)=F_{\mu}$. 
We shall make much use of the fact that the universal cover of $B F_{\mu}=\bigvee_{\mu} S^{1}$ is the contractible space with free $F_{\mu}$-action defined by the Cayley tree $E F_{\mu}=G_{\mu}$ of $F_{\mu}$, with vertices $g \in F_{\mu}$ and edges $\left(g, g z_{i}\right)\left(g \in F_{\mu}, 1 \leqslant i \leqslant \mu\right)$. The cellular chain complex $C\left(E F_{\mu}\right)=C\left(G_{\mu}\right)$ is the standard 1-dimensional f.g. free $\mathbb{Z}\left[F_{\mu}\right]$-module resolution of $\mathbb{Z}$

$$
0 \longrightarrow C_{1}\left(G_{\mu}\right)=\bigoplus_{i=1}^{\mu} \mathbb{Z}\left[F_{\mu}\right] \stackrel{d}{\longrightarrow} C_{0}\left(G_{\mu}\right)=\mathbb{Z}\left[F_{\mu}\right] \longrightarrow \mathbb{Z} \longrightarrow 0,
$$

the Mayer-Vietoris presentation with $d=\left(z_{1}-1 z_{2}-1 \ldots z_{\mu}-1\right)$.

The exterior $W$ of an $n$-dimensional link $\ell: \bigsqcup_{\mu} S^{n} \subset S^{n+2}$ is homotopy equivalent to the complement $S^{n+2} \backslash \ell\left(\bigsqcup_{\mu} S^{n}\right)$, so that

$$
\begin{aligned}
H_{*}(W) & =H_{*}\left(S^{n+2} \backslash \ell\left(\bigsqcup_{\mu} S^{n}\right)\right) \\
& =H^{n+2-*}\left(S^{n+2}, \ell\left(\bigsqcup_{\mu} S^{n}\right)\right)=H^{n+1-*}\left(\bigsqcup_{\mu} S^{n}\right) \quad(* \neq 0, n+2)
\end{aligned}
$$

by Alexander duality. The homology groups $H_{*}(W), H_{*}\left(W_{0}\right)$ are thus the same:

$$
H_{r}(W)=H_{r}\left(W_{0}\right)= \begin{cases}\mathbb{Z} & \text { if } r=0 \\ \bigoplus_{\mu} \mathbb{Z} & \text { if } r=1 \\ \bigoplus_{\mu-1} \mathbb{Z} & \text { if } r=n+1 \\ 0 & \text { otherwise. }\end{cases}
$$

The homotopy groups $\pi_{*}(W), \pi_{*}\left(W_{0}\right)$ are in general not the same, on account of linking. By Smythe [43] and Gutierrez [22] $\ell$ is a boundary link if and only if there exists a surjection $\pi_{1}(W) \rightarrow \pi_{1}\left(W_{0}\right)=F_{\mu}$ sending the meridians $m_{1}, m_{2}, \ldots, m_{\mu}: S^{1} \subset$ $W$ around the $\mu$ components $\ell_{1}, \ell_{2}, \ldots, \ell_{\mu}: S^{n} \subset S^{n+2}$ of $\ell$ to the generators $z_{1}, z_{2}, \ldots, z_{\mu} \in F_{\mu}$. We shall only be considering boundary links $\ell$ with a particular choice of such a surjection $\pi_{1}(W) \rightarrow F_{\mu}$, the $F_{\mu}$-links of Cappell and Shaneson [9]. For any such $\ell$ there exists a map $c: W \rightarrow W_{0}$ which induces a surjection $c_{*}: \pi_{1}(W) \rightarrow \pi_{1}\left(W_{0}\right)$ and isomorphisms $c_{*}: H_{*}(W) \cong H_{*}\left(W_{0}\right)$. Let $\widetilde{W}=c^{*} \widetilde{W}_{0}$ be the pullback $F_{\mu}$-cover of $W$, with a f.g. free $\mathbb{Z}\left[F_{\mu}\right]$-module cellular chain complex $C(\widetilde{W})$. An $F_{\mu}$-equivariant lift $\widetilde{c}: \widetilde{W} \rightarrow \widetilde{W}_{0}$ of $c$ induces a $\mathbb{Z}\left[F_{\mu}\right]$-module chain map $\tilde{c}: C(\widetilde{W}) \rightarrow C\left(\widetilde{W}_{0}\right)$ and a $\mathbb{Z}$-module chain equivalence $c: C(W) \rightarrow C\left(W_{0}\right)$. A $\mu-$ component Seifert surface $V=V_{1} \sqcup V_{2} \sqcup \ldots \sqcup V_{\mu} \subset S^{n+2}$ for $\ell$ has a neighbourhood $V \times[-1,1] \subset S^{n+2}$, with $V=V \times\{0\}$. The $F_{\mu}$-cover $\widetilde{W}$ can be constructed from $F_{\mu}$ copies of $S^{n+2} \backslash V$, glued together using the inclusions $f_{i}^{+}, f_{i}^{-}: V_{i} \rightarrow S^{n+2} \backslash V$ 
defined by

$$
f_{i}^{ \pm}\left(v_{i}\right)=\left(v_{i}, \pm 1\right) \in V \times[-1,1] \subset S^{n+2} .
$$

It follows that $C(\widetilde{W})$ has a f.g. free $\mathbb{Z}\left[F_{\mu}\right]$-module Mayer-Vietoris presentation

$$
0 \longrightarrow \bigoplus_{i=1}^{\mu} C\left(V_{i}\right)\left[F_{\mu}\right] \stackrel{f}{\longrightarrow} C\left(S^{n+2} \backslash V\right)\left[F_{\mu}\right] \longrightarrow C(\widetilde{W}) \longrightarrow 0
$$

with $f=f^{+}{ }_{z-f^{-}}=\left(f_{1}^{+} z_{1}-f_{1}^{-} \ldots f_{\mu}^{+} z_{\mu}-f_{\mu}^{-}\right)$.

\section{Seifert and Blanchfield modules}

There are four fundamental notions in our abstract version for any ring $A$ of the Seifert and Blanchfield modules of $\mu$-component boundary links:

(i) A Seifert A-module is a triple

$$
\left(P, e,\left\{\pi_{i}\right\}\right)=\left(A \text {-module, endomorphism, }\left\{\pi_{i}\right\}\right)
$$

where $\left\{\pi_{i}: P \rightarrow P\right\}$ is a system of idempotents expressing $P$ as a $\mu$-fold direct sum, with

$$
\begin{aligned}
\pi_{i}: P=P_{1} \oplus P_{2} \oplus \cdots \oplus P_{\mu} & \rightarrow P \\
\left(x_{1}, x_{2}, \ldots, x_{\mu}\right) & \mapsto\left(0, \ldots, 0, x_{i}, 0, \ldots, 0\right) .
\end{aligned}
$$

Let $\operatorname{Sei}_{\infty}(A)$ be the category of Seifert $A$-modules. A Seifert $A$-module $\left(P, e,\left\{\pi_{i}\right\}\right)$ is f.g. projective if $P$ is a f.g. projective $A$-module. Let $\operatorname{Sei}(A) \subset$ $\mathcal{S}_{e i_{\infty}}(A)$ be the full subcategory of the f.g. projective Seifert $A$-modules.

(ii) A Blanchfield $A\left[F_{\mu}\right]$-module $M$ is an $A\left[F_{\mu}\right]$-module such that

$$
\operatorname{Tor}_{*}^{A\left[F_{\mu}\right]}(A, M)=0,
$$

regarding $A$ as a right $A\left[F_{\mu}\right]$-module via the augmentation map

$$
\epsilon: A\left[F_{\mu}\right] \rightarrow A ; z_{i} \mapsto 1 .
$$

Let $\mathcal{B} l a_{\infty}(A)$ be the category of Blanchfield $A\left[F_{\mu}\right]$-modules. In Section 3.2 Blanchfield $A\left[F_{\mu}\right]$-modules will be identified with the $F_{\mu}$-link modules in the sense of Sheiham [41], that is $A\left[F_{\mu}\right]$-modules $M$ which admit an $A\left[F_{\mu}\right]-$ module presentation

$$
0 \longrightarrow P\left[F_{\mu}\right] \stackrel{d}{\longrightarrow} Q\left[F_{\mu}\right] \longrightarrow M \longrightarrow 0
$$


for $A$-modules $P, Q$ with the augmentation $\epsilon(d): P \rightarrow Q$ an $A$-module isomorphism. Thus $\mathcal{B} l a_{\infty}(A)$ is just the $F_{\mu}$-link module category $\mathcal{F} l k_{\infty}(A)$ of [41]. A Blanchfield $A\left[F_{\mu}\right]$-module $M$ has homological dimension 1 (or h.d. 1 for short) if it has a 1-dimensional f.g. projective $A\left[F_{\mu}\right]$-module resolution

$$
0 \longrightarrow K \stackrel{d}{\longrightarrow} L \longrightarrow M \longrightarrow 0
$$

with (necessarily) $\epsilon(d)=1 \otimes d: A \otimes_{A\left[F_{\mu}\right]} K \rightarrow A \otimes_{A\left[F_{\mu}\right]} L$ an $A$-module isomorphism. Let $\mathcal{B l a}(A) \subset \mathcal{B} l a_{\infty}(A)$ be the full subcategory of the h.d. 1 Blanchfield $A\left[F_{\mu}\right]$-modules. Let $\mathcal{F} l k(A) \subset \mathcal{B} l a(A)$ be the full subcategory of the h.d. 1 Blanchfield modules $M$ which admit a 1-dimensional induced f.g. projective $A\left[F_{\mu}\right]$-module resolution

$$
0 \longrightarrow P\left[F_{\mu}\right] \stackrel{d}{\longrightarrow} Q\left[F_{\mu}\right] \longrightarrow M \longrightarrow 0
$$

with $P, Q$ f.g. projective $A$-modules. As in [41] the objects of $\mathcal{F} l k(A)$ will be called h.d. $1 F_{\mu}$-link modules.

(iii) The covering of a Seifert $A$-module $\left(P, e,\left\{\pi_{i}\right\}\right)$ is the Blanchfield $A\left[F_{\mu}\right]-$ module

$$
B\left(P, e,\left\{\pi_{i}\right\}\right)=\operatorname{coker}\left(1-e+e z: P\left[F_{\mu}\right] \rightarrow P\left[F_{\mu}\right]\right)
$$

with $z=\sum_{i=1}^{\mu} \pi_{i} z_{i}: P\left[F_{\mu}\right] \rightarrow P\left[F_{\mu}\right]$, defining functors

$$
B_{\infty}: \mathcal{S e i}_{\infty}(A) \rightarrow \mathcal{B} l a_{\infty}(A), B: \operatorname{Sei}(A) \rightarrow \mathcal{F} l k(A) .
$$

(iv) A Seifert $A$-module $\left(P, e,\left\{\pi_{i}\right\}\right)$ is primitive if $B\left(P, e,\left\{\pi_{i}\right\}\right)=0$. Let

$$
\operatorname{Prim}_{\infty}(A)=\operatorname{ker}\left(B_{\infty}: \mathcal{S e i}_{\infty}(A) \rightarrow \mathcal{B} \operatorname{la}_{\infty}(A)\right)
$$

be the full subcategory of $\operatorname{Sei}_{\infty}(A)$ with objects the primitive Seifert $A$-modules, and let

$$
\operatorname{Prim}(A)=\operatorname{ker}(B: \operatorname{Sei}(A) \rightarrow \mathcal{F} l k(A)) \subset \mathcal{S e i}(A)
$$

be the full subcategory of $\operatorname{Sei}(A)$ with objects the primitive f.g. projective Seifert $A$-modules.

\section{Simple boundary links}

The motivational examples of f.g. projective Seifert $\mathbb{Z}$-modules and h.d. $1 F_{\mu}$-link $\mathbb{Z}\left[F_{\mu}\right]$-modules come from the $(2 q-1)$-dimensional $\mu$-component boundary links 
$\ell: \bigsqcup_{\mu} S^{2 q-1} \subset S^{2 q+1}$ which are simple, meaning that the exterior $W$ has homotopy groups

$$
\pi_{r}(W)= \begin{cases}F_{\mu} & \text { if } r=1 \\ 0 & \text { if } 2 \leqslant r \leqslant q-1,\end{cases}
$$

so that the universal cover $\widetilde{W}$ is $(q-1)$-connected. These conditions are equivalent to the existence of a $\mu$-component Seifert surface $V=V_{1} \sqcup V_{2} \sqcup \cdots \sqcup V_{\mu}$ with each component $V_{i}(q-1)$-connected:

$$
\pi_{r}\left(V_{i}\right)=0 \quad(1 \leqslant i \leqslant \mu, 1 \leqslant r \leqslant q-1) .
$$

The homology of the Seifert surface defines a f.g. projective (actually f.g. free) Seifert $\mathbb{Z}$-module $\left(P, e,\left\{\pi_{i}\right\}\right)$, with

and

$$
\pi_{i}=0 \oplus \cdots \oplus 0 \oplus 1 \oplus 0 \oplus \cdots \oplus 0: P=\bigoplus_{i=1}^{\mu} H_{q}\left(V_{i}\right) \rightarrow P=\bigoplus_{i=1}^{\mu} H_{q}\left(V_{i}\right)
$$

$$
\begin{aligned}
& e=\left(f_{1}^{+} f_{2}^{+} \ldots f_{\mu}^{+}\right): P=H_{q}(V)=\bigoplus_{i=1}^{\mu} H_{q}\left(V_{i}\right) \longrightarrow \\
& H_{q}\left(S^{2 q+1} \backslash V\right)=H^{q}(V)=H_{q}(V)=P
\end{aligned}
$$

the endomorphism induced by the inclusions $f_{i}^{+}: V_{i} \rightarrow S^{2 q+1} \backslash V$, identifying

$$
H_{q}\left(S^{2 q+1} \backslash V\right)=H^{q}(V)
$$

by Alexander duality and $H^{q}(V)=H_{q}(V)$ by Poincaré duality. The covering of $\left(P, e,\left\{\pi_{i}\right\}\right)$ is the h.d. $1 F_{\mu}$-link $\mathbb{Z}\left[F_{\mu}\right]$-module

$$
B\left(P, e,\left\{\pi_{i}\right\}\right)=H_{q}(\widetilde{W})
$$

defined by the homology of the $F_{\mu}$-cover $\widetilde{W}$ of the exterior $W$. The f.g. projective Seifert $\mathbb{Z}$-module $\left(P, e,\left\{\pi_{i}\right\}\right)$ is primitive if and only if $H_{q}(\widetilde{W})=0$; for $q \geqslant 2$ this is the case if and only if $\ell$ is unlinked (Gutierrez [22]).

\section{Blanchfield $=$ Seifert $/$ primitive}

Section 3 uses algebraic transversality to prove that every h.d. $1 F_{\mu}$-link module $M$ is isomorphic to the covering $B\left(P, e,\left\{\pi_{i}\right\}\right)$ of a f.g. projective Seifert $A$-module $\left(P, e,\left\{\pi_{i}\right\}\right)$, and that morphisms of h.d. $1 F_{\mu}$-link modules can be expressed as fractions of morphisms of f.g. projective Seifert $A$-modules.

The algebraic relation between Seifert $A$-modules and Blanchfield $A\left[F_{\mu}\right]$-modules for $A=\mathbb{Z}$ was first investigated systematically in the knot case $\mu=1$, by Levine [25; 
26] and Trotter [46], and for the link case $\mu \geqslant 1$ by Farber [13; 14] and Sheiham [41]. In particular, [41] expressed the Blanchfield module category $\mathcal{B} l a_{\infty}(A)=\mathcal{F} l k_{\infty}(A)$ as the quotient of the Seifert $A$-module category $\mathcal{S e i}_{\infty}(A)$ by the primitive Seifert $A$-module subcategory $\operatorname{Prim}_{\infty}(A)$, as we now recall.

Let $\mathcal{A}$ be an abelian category. By definition, a Serre subcategory $\mathcal{C} \subset \mathcal{A}$ is a non-empty full subcategory such that for every exact sequence in $\mathcal{A}$

$$
0 \rightarrow M^{\prime} \rightarrow M \rightarrow M^{\prime \prime} \rightarrow 0
$$

$M$ is an object in $\mathcal{C}$ if and only if $M^{\prime}, M^{\prime \prime}$ are objects in $\mathcal{C}$. Gabriel [17] defined the quotient abelian category $\mathcal{A} / \mathcal{C}$ with the same objects as $\mathcal{A}$ but different morphisms: if $M, N$ are objects in $\mathcal{A}$ then

$$
\operatorname{Hom}_{\mathcal{A} / \mathcal{C}}(M, N)=\lim _{\longrightarrow} \operatorname{Hom}_{\mathcal{A}}\left(M^{\prime}, N^{\prime \prime}\right)
$$

with the direct limit taken over all the exact sequences in $\mathcal{A}$

$$
0 \rightarrow M^{\prime} \rightarrow M \rightarrow M^{\prime \prime} \rightarrow 0,0 \rightarrow N^{\prime} \rightarrow N \rightarrow N^{\prime \prime} \rightarrow 0
$$

with $M^{\prime \prime}, N^{\prime}$ objects in $\mathcal{C}$. The canonical functor $F: \mathcal{A} \rightarrow \mathcal{A} / \mathcal{C} ; A \mapsto A$ sends each object $C$ in $\mathcal{C}$ to $F(C)=0$, and has the universal property that for any exact functor $G: \mathcal{A} \rightarrow \mathcal{B}$ such that $G(C)=0$ for all objects in $\mathcal{C}$ there exists a unique functor $\bar{G}: \mathcal{A} / \mathcal{C} \rightarrow \mathcal{B}$ such that $\bar{G} F=G$. In particular, if $\mathcal{B}$ is an exact category and $G: \mathcal{A} \rightarrow \mathcal{B}$ is an exact functor then the full subcategory $\mathcal{C} \subset \mathcal{A}$ with objects $C$ such that $G(C)=0$ is a Serre subcategory, and there is induced a functor $\bar{G}: \mathcal{A} / \mathcal{C} \rightarrow \mathcal{B} ; A \mapsto G(A)$ such that $G=\bar{G} F$.

By definition, a category is small if the class of morphisms is a set. In order to avoid set-theoretic difficulties we shall only be dealing with categories which are essentially small, ie equivalent to a small category.

Let $\mathcal{A}$ be an essentially small category, and let $\Sigma$ be a set of morphisms in $\mathcal{A}$, e.g. the morphisms of a subcategory. A category of fractions $\Sigma^{-1} \mathcal{A}$ is a category with a universally $\Sigma$-inverting functor $F: \mathcal{A} \rightarrow \Sigma^{-1} \mathcal{A}$, meaning that:

(i) $F$ sends each $f \in \Sigma$ to an isomorphism $F(f)$ in $\Sigma^{-1} \mathcal{A}$,

(ii) for any functor $G: \mathcal{A} \rightarrow \mathcal{B}$ which sends each $f \in \Sigma$ to an isomorphism $G(f)$ there exists a unique functor $\bar{G}: \Sigma^{-1} \mathcal{A} \rightarrow \mathcal{B}$ such that $\bar{G} F=G$.

An essentially small category of fractions $\Sigma^{-1} \mathcal{A}$ exists, with the same objects as $\mathcal{A}$, and such a category is unique up to isomorphism (Gabriel and Zisman [18], Borceux 
[7, 5.2.2]). For example, if $\mathcal{A}$ is an abelian category and $\mathcal{C} \subset \mathcal{A}$ is a Serre subcategory, then

$$
\mathcal{A} / \mathcal{C}=\Sigma^{-1} \mathcal{A}
$$

is a category of fractions inverting the set $\Sigma$ of morphisms $f$ in $\mathcal{A}$ with $\operatorname{ker}(f)$ and $\operatorname{coker}(f)$ in $\mathcal{C}$.

An $A\left[F_{\mu}\right]$-module $M$ is Blanchfield if and only if the $A$-module morphism

$$
\gamma: \bigoplus_{\mu} M \rightarrow M ;\left(m_{1}, m_{2}, \ldots, m_{\mu}\right) \mapsto \sum_{i=1}^{\mu}\left(z_{i}-1\right) m_{i}
$$

is an isomorphism, called the Sato isomorphism (after [37], the case $A=\mathbb{Z}$ ). As in Sheiham [41], for any Blanchfield $A\left[F_{\mu}\right]$-module $M$ use the $A$-module morphisms

$$
\begin{aligned}
& p_{i}: \bigoplus_{\mu} M \rightarrow M ; \quad\left(m_{1}, m_{2}, \ldots, m_{\mu}\right) \mapsto m_{i}, \\
& \omega: \bigoplus_{\mu} M \rightarrow M ; \quad\left(m_{1}, m_{2}, \ldots, m_{\mu}\right) \mapsto \sum_{i=1}^{\mu} m_{i}, \\
& \pi_{i}=\gamma p_{i} \gamma^{-1}: M \rightarrow M, \\
& e=\omega \gamma^{-1}: M \rightarrow M
\end{aligned}
$$

to define a Seifert $A$-module $U(M)=\left(M, e,\left\{\pi_{i}\right\}\right)$.

The categories $\mathcal{P r i m}_{\infty}(A), \mathcal{S} e i_{\infty}(A)$ are abelian, while $\mathcal{B} l_{\infty}(A)$ is in general only exact. The covering functor $B_{\infty}: \mathcal{S e i}_{\infty}(A) \rightarrow \mathcal{B} a_{\infty}(A)$ was shown in $[41,5.2]$ to be exact, so that $\operatorname{Prim}_{\infty}(A) \subset \mathcal{S e i}_{\infty}(A)$ is a Serre subcategory. Thus if $\Xi_{\infty}$ is the set of morphisms $f$ in $\mathcal{S e i}_{\infty}(A)$ such that $B(f)$ is an isomorphism in $\mathcal{B l a} a_{\infty}(A)$, or equivalently $\operatorname{ker}(f)$ and $\operatorname{coker}(f)$ are in $\operatorname{Prim}_{\infty}(A)$, then

$$
\mathcal{S}_{e i_{\infty}}(A) / \mathcal{P r i m}_{\infty}(A)=\Xi_{\infty}^{-1} \mathcal{S e i}_{\infty}(A) .
$$

The induced exact functor $\bar{B}_{\infty}: \mathcal{S e i}_{\infty}(A) / \mathcal{P} \operatorname{rim}_{\infty}(A) \rightarrow \mathcal{B} l a_{\infty}(A)$ is such that

$$
B_{\infty}: \mathcal{S}_{e i_{\infty}}(A) \rightarrow \mathcal{S}_{e i_{\infty}}(A) / \mathcal{P r i m}_{\infty}(A) \stackrel{\bar{B}_{\infty}}{\longrightarrow} \mathcal{B l a}_{\infty}(A)
$$

and has the universal property of inverting $\Xi_{\infty}$. The functor $\bar{B}_{\infty}$ was shown to be an equivalence in $[41,5.15]$ using the fact that the functor

$$
U_{\infty}: \mathcal{B} l a_{\infty}(A) \rightarrow \mathcal{S}_{e i_{\infty}}(A) ; M \mapsto U(M)
$$

is right adjoint to $B$ : for any Seifert $A$-module $V$ there is a natural isomorphism

$$
\operatorname{Hom}_{\mathcal{B} l a_{\infty}(A)}(B(V), M) \cong \operatorname{Hom}_{\mathcal{S} e i_{\infty}(A)}(V, U(M)) .
$$


The functor $U_{\infty}$ is fully faithful, allowing $\mathcal{B} l a_{\infty}(A)$ to be regarded as a full subcategory of $\mathcal{S}_{e i_{\infty}}(A)$. By $[41,5.15] U_{\infty}$ induces a functor

$$
\bar{U}_{\infty}: \mathcal{B l a}_{\infty}(A) \rightarrow \mathcal{S e i}_{\infty}(A) / \mathcal{P r i m}_{\infty}(A)
$$

which is an equivalence inverse to $\bar{B}_{\infty}$. Thus up to equivalence

$$
\mathcal{S}_{e i_{\infty}}(A) / \mathcal{P r i m}_{\infty}(A)=\Xi_{\infty}^{-1} \mathcal{S e i}_{\infty}(A)=\mathcal{B} l a_{\infty}(A)
$$

The categories $\mathcal{P} \operatorname{rim}(A), \mathcal{S e i}(A), \mathcal{F} l k(A), \mathcal{B} l a(A)$ are exact but not in general abelian. As in [41] let $\operatorname{Sei}(A) / \mathcal{P r i m}_{\infty}(A) \subset \mathcal{S e i}_{\infty}(A) / \mathcal{P r i m}_{\infty}(A)$ be the full subcategory with objects in $\operatorname{Sei}(A)$. The equivalence

$$
\bar{B}_{\infty}: \operatorname{Sei}_{\infty}(A) / \operatorname{Prim}_{\infty}(A) \stackrel{\approx}{\longrightarrow} \mathcal{B l a}_{\infty}(A)
$$

was shown in $[41,5.17]$ to restrict to an equivalence of exact sequences

$$
\bar{B}: \operatorname{Sei}(A) / \operatorname{Prim}_{\infty}(A) \stackrel{\approx}{\longrightarrow} \mathcal{F} l k(A)
$$

with

$$
B: \operatorname{Sei}(A) \rightarrow \operatorname{Sei}(A) / \operatorname{Prim}_{\infty}(A) \stackrel{\bar{B}}{\underset{\approx}{*}} \mathcal{F} l k(A) .
$$

From the construction of $\mathcal{S}_{e i_{\infty}}(A) / \mathcal{P r i m}_{\infty}(A)$ a morphism in $\operatorname{Sei}(A) / \mathcal{P r i m}_{\infty}(A)$ may involve objects in $\mathcal{S e i}_{\infty}(A)$ which are not in $\mathcal{S} e i(A)$, so that the equivalence $\bar{B}$ cannot be used to relate the algebraic $K$-theories of $\operatorname{Sei}(A)$ and $\mathcal{F} l k(A)$.

A category of fractions $\Sigma^{-1} \mathcal{A}$ has a left calculus of fractions if:

(i) $(1: A \rightarrow A) \in \Sigma$ for every object $A$ in $\mathcal{A}$,

(ii) if $(s: A \rightarrow B),(t: B \rightarrow C) \in \Sigma$ then $(t s: A \rightarrow C) \in \Sigma$,

(iii) for any $f: A \rightarrow B$ in $\mathcal{A}$ and $s: A \rightarrow D$ in $\Sigma$ there exist $g: D \rightarrow C$ in $\mathcal{A}$ and $t: B \rightarrow C$ in $\Sigma$ such that $t f=g s: A \rightarrow C$,

(iv) for any $f, g: A \rightarrow B$ in $\mathcal{A}$ and $s: D \rightarrow A$ in $\Sigma$ with $f s=g s: D \rightarrow B$ there exists $(t: B \rightarrow C) \in \Sigma$ with $t f=\operatorname{tg}: A \rightarrow C$.

It then follows that a morphism $A \rightarrow B$ in $\Sigma^{-1} \mathcal{A}$ can be regarded as an equivalence class $s^{-1} f$ of pairs $(f: A \rightarrow C, s: B \rightarrow C$ ) of morphisms in $\mathcal{A}$ with $s \in \Sigma$, where

$$
\begin{aligned}
(f, s) \sim\left(f^{\prime}, s^{\prime}\right) & \text { if there exist morphisms } g: C \rightarrow D, g^{\prime}: C^{\prime} \rightarrow D \text { in } \mathcal{A} \\
& \text { with }\left(g s=g^{\prime} s^{\prime}: B \rightarrow D\right) \in \Sigma \text { and } g f=g^{\prime} f^{\prime}: A \rightarrow D
\end{aligned}
$$

so that

$$
s^{-1} f=(g s)^{-1}(g f)=\left(g^{\prime} s^{\prime}\right)^{-1}\left(g^{\prime} f^{\prime}\right)=s^{\prime-1} f^{\prime}: A \rightarrow B \text { in } \Sigma^{-1} \mathcal{A} .
$$


Let $\Xi$ be the set of morphisms $f$ in $\operatorname{Sei}(A)$ such that $B(f)$ is an isomorphism in $\mathcal{F} l k(A)$, or equivalently such that $\operatorname{ker}(f)$ and $\operatorname{coker}(f)$ are in $\operatorname{Prim}_{\infty}(A)$. In Section 3 we shall prove:

Theorem B (i) The category of fractions $\Xi^{-1} \operatorname{Sei}(A)$ has a left calculus of fractions, and the covering functor $B: \mathcal{S e i}(A) \rightarrow \mathcal{F} l k(A)$ induces an equivalence of exact categories

$$
\bar{B}: \Xi^{-1} \operatorname{Sei}(A) \stackrel{\approx}{\longrightarrow} \mathcal{F} l k(A) .
$$

(ii) The h.d. 1 Blanchfield $A\left[F_{\mu}\right]$-module category $\operatorname{Bla}(A)$ is the idempotent completion of the h.d. $1 F_{\mu}$-link module category $\mathcal{F} l k(A)$.

The key step in the proof of Theorem B (i) is the use of the algebraic transversality Theorem A to verify that for any h.d. $1 F_{\mu}$-link module $M$ the Seifert $A$-module $U(M)$ is a direct limit of morphisms in $\Xi$.

\section{Primitive $=$ near-projection}

Section 4 gives an intrinsic characterization of the primitive f.g. projective Seifert $A$-modules $\left(P, e,\left\{\pi_{i}\right\}\right)$ as generalized near-projections.

An endomorphism $e: P \rightarrow P$ of an $A$-module $P$ is nilpotent if $e^{N}=0$ for some $N \geqslant 0$.

An endomorphism $e: P \rightarrow P$ is a near-projection if $e(1-e): P \rightarrow P$ is nilpotent (Lück and Ranicki [28]).

In Section 4 we shall prove:

Theorem C A f.g. projective Seifert $A$-module $\left(P, e,\left\{\pi_{i}\right\}\right)$ is primitive if and only if it can be expressed as

$$
\left(P, e,\left\{\pi_{i}\right\}\right)=\left(P^{+} \oplus P^{-},\left(\begin{array}{cc}
e^{++} & e^{+-} \\
e^{-+} & e^{--}
\end{array}\right),\left\{\pi_{i}^{+}\right\} \oplus\left\{\pi_{i}^{-}\right\}\right)
$$

and the $2 \mu$-component Seifert $A$-module

$$
\left(P^{\prime}, e^{\prime}, \pi^{\prime}\right)=\left(P^{+} \oplus P^{-},\left(\begin{array}{cc}
e^{++} & -e^{+-} \\
e^{-+} & 1-e^{--}
\end{array}\right),\left\{\pi_{i}^{+}\right\} \oplus\left\{\pi_{i}^{-}\right\}\right)
$$

is such that $e^{\prime} z^{\prime}: P^{\prime}\left[F_{2 \mu}\right] \rightarrow P^{\prime}\left[F_{2 \mu}\right]$ is nilpotent, with $F_{2 \mu}$ the free group on $2 \mu$ generators $z_{1}^{\prime}, \ldots, z_{2 \mu}^{\prime}$. 
For $\mu=1$ the condition for a f.g. projective Seifert $A$-module $\left(P, e,\left\{\pi_{i}\right\}\right)$ to be primitive is just that $e$ be a near-projection. For $\mu=1$ Theorem $C$ is just the result of Bass, Heller and Swan [5] that $1-e+e z: P\left[z, z^{-1}\right] \rightarrow P\left[z, z^{-1}\right]$ is an $A\left[z, z^{-1}\right]-$ module isomorphism if and only if $e$ is a near-projection, if and only if $(P, e)=$ $\left(P^{+}, e^{++}\right) \oplus\left(P^{-}, e^{--}\right)$with $e^{++}: P^{+} \rightarrow P^{+}$and $1-e^{--}: P^{-} \rightarrow P^{-}$nilpotent.

\section{Algebraic $K$-theory}

Section 5 obtains results on the algebraic $K$-theory of $A\left[F_{\mu}\right], \mathcal{P} \operatorname{rim}(A), \operatorname{Sei}(A)$, $\mathcal{F} l k(A)$ and $\mathcal{B} l a(A)$, using the algebraic $K$-theory noncommutative localization exact sequences of Schofield [39] and Neeman-Ranicki [30; 31].

The class group $K_{0}(\mathcal{E})$ of an exact category $\mathcal{E}$ is the Grothendieck group with one generator $[M]$ for each object $M$ in $\mathcal{E}$, and one relation $[K]-[L]+[M]=0$ for each exact sequence in $\mathcal{E}$

$$
0 \rightarrow K \rightarrow L \rightarrow M \rightarrow 0 .
$$

The algebraic $K$-groups $K_{n}(\mathcal{E})$ are defined by Quillen [32] for $n \geqslant 1$ and by Schlichting [38] for $n \leqslant-1$. Write

$$
\begin{aligned}
\operatorname{Prim}_{*}(A) & =K_{*}(\mathcal{P} \operatorname{Prim}(A)), & & \operatorname{Sei}_{*}(A)=K_{*}(\mathcal{S e i}(A)), \\
\operatorname{Bla}_{*}(A) & =K_{*}(\mathcal{B l a}(A)), & & \operatorname{Flk}_{*}(A)=K_{*}(\mathcal{F} l k(A)),
\end{aligned}
$$

noting that $\mathrm{Bla}_{n}(A)=\mathrm{Flk}_{n}(A)$ for $n \neq 0$.

Theorem D (i) The algebraic $K$-groups of $A\left[F_{\mu}\right]$ split as

$$
K_{*}\left(A\left[F_{\mu}\right]\right)=K_{*}(A) \oplus \bigoplus_{\mu} K_{*-1}(A) \oplus \widetilde{\operatorname{Prim}}_{*-1}(A)
$$

(ii) The sequence of functors

$$
\operatorname{Prim}(A) \longrightarrow \operatorname{Sei}(A) \stackrel{B}{\longrightarrow} \operatorname{Bla}(A)
$$

induces a long exact sequence of algebraic $K$-groups

$$
\cdots \rightarrow \operatorname{Prim}_{n}(A) \rightarrow \operatorname{Sei}_{n}(A) \stackrel{B}{\longrightarrow} \operatorname{Bla}_{n}(A) \rightarrow \operatorname{Prim}_{n-1}(A) \rightarrow \cdots
$$

with

$$
\operatorname{im}\left(B: \operatorname{Sei}_{0}(A) \rightarrow \operatorname{Bla}_{0}(A)\right)=\operatorname{Flk}_{0}(A) \subseteq \operatorname{Bla}_{0}(A) .
$$


(iii) The exact sequence in (ii) splits as a direct sum of exact sequences

$$
\begin{aligned}
& \cdots \rightarrow \underset{2 \mu}{\bigoplus} K_{n}(A) \rightarrow \underset{\mu}{\bigoplus} K_{n}(A) \stackrel{0}{\rightarrow} \bigoplus_{\mu} K_{n-1}(A) \rightarrow \underset{2 \mu}{\bigoplus} K_{n-1}(A) \rightarrow \cdots, \\
& \cdots \rightarrow \underset{\operatorname{Prim}_{n}}{ }(A) \rightarrow \underset{\operatorname{Sei}_{n}}{ }(A) \rightarrow{\widetilde{\operatorname{Bla}_{n}}}_{n}(A) \rightarrow \widetilde{\operatorname{Prim}}_{n-1}(A) \rightarrow \cdots .
\end{aligned}
$$

For $\mu=1 \mathcal{P} \operatorname{rim}(A)$ is the exact category of f.g. projective $A$-modules $P$ with a near-projection $e: P \rightarrow P$, which is equivalent to the product $\mathcal{N} i l(A) \times \mathcal{N} i l(A)$ of two copies of the exact category $\mathcal{N} i l(A)$ of f.g. projective $A$-modules $P$ with a nilpotent endomorphism $e: P \rightarrow P$, and

$$
\begin{aligned}
\operatorname{Prim}_{*}(A) & =K_{*}(\mathcal{P} \operatorname{rim}(A))=\operatorname{Nil}_{*}(A) \oplus \operatorname{Nil}_{*}(A), \\
\widetilde{N i l}_{*}(A) & =K_{*}(\mathcal{N i l}(A))=K_{*}(A) \oplus \widetilde{\mathrm{Nil}}_{*}(A), \\
\widetilde{\operatorname{Prim}}_{*}(A) & =\widetilde{\operatorname{Nil}_{*}}(A) \oplus \widetilde{\operatorname{Nil}}_{*}(A) .
\end{aligned}
$$

Thus for $\mu=1$ Theorem D (i) is just the splitting theorem of Bass, Heller and Swan [5], [4] for $K_{1}\left(A\left[z, z^{-1}\right]\right)$ and its generalization to the higher $K$-groups

$$
K_{*}\left(A\left[z, z^{-1}\right]\right)=K_{*}(A) \oplus K_{*-1}(A) \oplus \widetilde{\operatorname{Nil}}_{*-1}(A) \oplus \widetilde{\operatorname{Nil}}_{*-1}(A) .
$$

Theorem D (ii)-(iii) is new even in the case $\mu=1$.

Let $\Sigma^{-1} A\left[F_{\mu}\right]$ be the noncommutative Cohn (ie universal) localization of $A\left[F_{\mu}\right]$ inverting the set $\Sigma$ of the morphisms of f.g. projective $A\left[F_{\mu}\right]$-modules which induce isomorphisms of f.g. projective $A$-modules under the augmentation $\epsilon: A\left[F_{\mu}\right] \rightarrow A$. The exact category $H\left(A\left[F_{\mu}\right], \Sigma\right)$ of h.d. $1 \Sigma$-torsion $A\left[F_{\mu}\right]$-modules is such that

$$
H\left(A\left[F_{\mu}\right], \Sigma\right)=\operatorname{Bla}(A), K_{*}\left(H\left(A\left[F_{\mu}\right], \Sigma\right)\right)=\operatorname{Bla}_{*}(A) .
$$

Theorem E (i) The localization exact sequence

$$
K_{1}\left(A\left[F_{\mu}\right]\right) \rightarrow K_{1}\left(\Sigma^{-1} A\left[F_{\mu}\right]\right) \rightarrow K_{0}\left(H\left(A\left[F_{\mu}\right], \Sigma\right)\right) \rightarrow K_{0}\left(A\left[F_{\mu}\right]\right) \rightarrow \cdots
$$

splits as a direct sum of the exact sequences

$$
\begin{aligned}
& K_{1}(A) \oplus \underset{\mu}{\bigoplus} K_{0}(A) \rightarrow K_{1}(A) \stackrel{0}{\rightarrow} \underset{\mu}{\bigoplus} K_{-1}(A) \rightarrow K_{0}(A) \oplus \underset{\mu}{\bigoplus_{\mu}} K_{-1}(A) \rightarrow \cdots, \\
& \widetilde{\operatorname{Prim}}_{0}(A) \rightarrow \widetilde{\operatorname{Sei}}_{0}(A) \rightarrow{\widetilde{\operatorname{Bla}_{0}}}(A) \rightarrow \underset{\operatorname{Prim}_{-1}}{ }(A) \rightarrow \cdots .
\end{aligned}
$$

(ii) If $\Sigma^{-1} A\left[F_{\mu}\right]$ is stably flat (ie if $\operatorname{Tor}_{*}^{A\left[F_{\mu}\right]}\left(\Sigma^{-1} A\left[F_{\mu}\right], \Sigma^{-1} A\left[F_{\mu}\right]\right)=0$ for $* \geqslant 1$ ) the exact sequences and the splitting in (i) extend to the left, involving the algebraic $K$-groups $K_{n}$ for $n \geqslant 2$, with

$$
K_{*}\left(\Sigma^{-1} A\left[F_{\mu}\right]\right)=K_{*}(A) \oplus \widetilde{\operatorname{Sei}}_{*-1}(A) .
$$


For $\mu=1 \mathcal{S} e i(A)$ is the exact category $\mathcal{E} n d(A)$ of f.g. projective $A$-modules $P$ with an endomorphism $e: P \rightarrow P$, and

$$
\operatorname{Sei}_{*}(A)=K_{*}(\mathcal{E} n d(A))=\operatorname{End}_{*}(A)=K_{*}(A) \oplus \widetilde{\operatorname{End}}_{*}(A) .
$$

The special case of Theorem E (i)

$$
K_{1}\left(\Sigma^{-1} A\left[z, z^{-1}\right]\right)=K_{1}(A) \oplus \widetilde{\operatorname{End}}_{0}(A)
$$

is the splitting theorem of Ranicki [33, 10.21].

We are grateful to Pere Ara, Warren Dicks, Marco Schlichting and the referee for helpful comments on the preprint version of the paper, which have led to various improvements. In particular, it was Pere Ara who pointed out that the Blanchfield $A\left[F_{\mu}\right]$-module category $\mathcal{B} l a_{\infty}(A)$ is the same as the $F_{\mu}$-link module category $\mathcal{F} l k_{\infty}(A)$ of [41].

\section{Combinatorial transversality for $F_{\mu}$-covers}

For $\mu \geqslant 1$ let $F_{\mu}=\left\langle z_{1}, z_{2}, \ldots, z_{\mu}\right\rangle$ be the free group with generators $z_{1}, z_{2}, \ldots, z_{\mu}$.

\section{$1.1 \quad F_{\mu}$-covers}

Definition 1.1 An $F_{\mu}$-cover of a space $W$ is a regular covering $p$ : $\widetilde{W} \rightarrow W$ with group of covering translations $F_{\mu}$.

A classifying space $B F_{\mu}$ for $F_{\mu}$-covers is a connected space such that

$$
\pi_{j}\left(B F_{\mu}\right)= \begin{cases}F_{\mu} & \text { if } j=1 \\ 0 & \text { if } j \geqslant 2 .\end{cases}
$$

The universal cover of $B F_{\mu}$ is an $F_{\mu}$-cover

$$
p_{\mu}: E F_{\mu}=\widetilde{B F}_{\mu} \rightarrow B F_{\mu}
$$

with $E F_{\mu}$ a contractible space with a free $F_{\mu}$-action.

Proposition 1.2 (i) Given an $F_{\mu}$-cover $p: \widetilde{W} \rightarrow W$ and a map $f: V \rightarrow W$ there is defined a pullback square

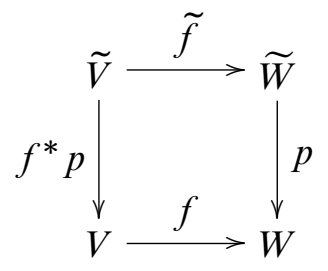


with

$$
\begin{aligned}
& \tilde{V}=f^{*} \widetilde{W}=\{(x, y) \in V \times \widetilde{W} \mid f(x)=p(y) \in W\}, \\
& f^{*} p: \widetilde{V} \rightarrow V ;(x, y) \mapsto x, \quad \tilde{f}: \widetilde{V} \rightarrow \widetilde{W} ;(x, y) \mapsto y
\end{aligned}
$$

such that $f^{*} p: \tilde{V} \rightarrow V$ is the pullback $F_{\mu}$-cover.

(ii) The $F_{\mu}$-covers $p: \widetilde{W} \rightarrow W$ of a space $W$ are classified by the homotopy classes of maps $c: W \rightarrow B F_{\mu}$ with

$$
\begin{aligned}
& \widetilde{W}=c^{*} E F_{\mu}=\left\{(x, y) \in W \times E F_{\mu} \mid c(x)=[y] \in B F_{\mu}\right\}, \\
& p(x, y)=c^{*} p_{\mu}(x, y)=x .
\end{aligned}
$$

For a connected space $W$ the homotopy classes of maps $c: W \rightarrow B F_{\mu}$ are in one-one correspondence with the morphisms $c_{*}: \pi_{1}(W) \rightarrow F_{\mu}$; the connected $F_{\mu}$-covers $\widetilde{W}$ correspond to surjections $c_{*}: \pi_{1}(W) \rightarrow F_{\mu}$.

Proof Standard.

\subsection{The Cayley tree $G_{\mu}$}

We shall be working with the following explicit constructions of $B F_{\mu}$ and $E F_{\mu}$, as well as the Cayley tree of $F_{\mu}$ :

Definition 1.3 The Cayley tree $G_{\mu}$ is the tree with vertex set

$$
G_{\mu}^{(0)}=F_{\mu}
$$

and edge set

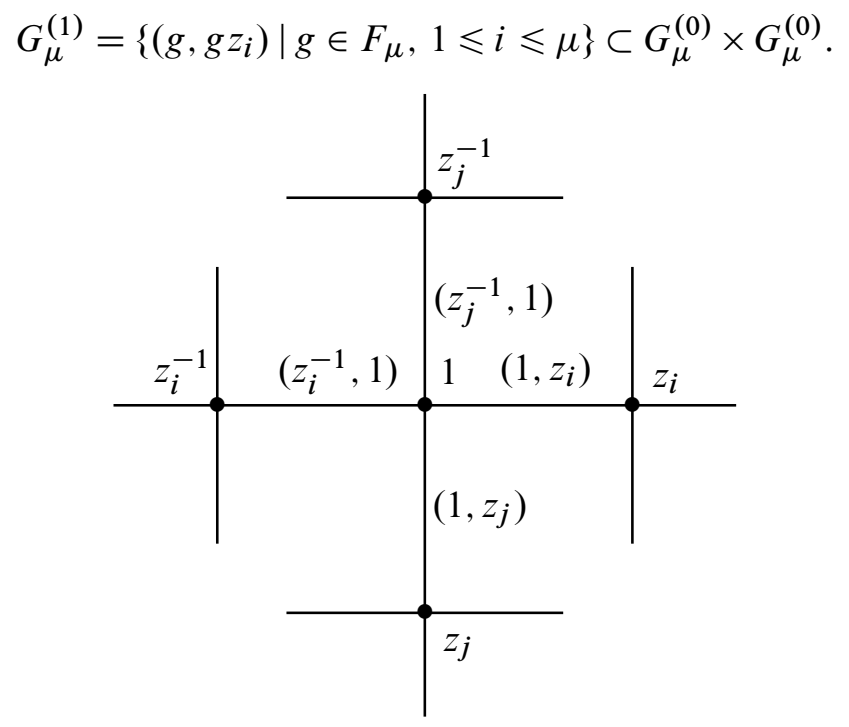


Define a transitive $F_{\mu}$-action on $G_{\mu}$

$$
F_{\mu} \times G_{\mu} \rightarrow G_{\mu} ;(g, x) \mapsto g x
$$

with quotient the one-point union of $\mu$ circles

$$
G_{\mu} / F_{\mu}=B F_{\mu}=\bigvee_{\mu} S^{1}
$$

Let

$$
I_{\mu}=\bigcup_{i=1}^{\mu}\left[e_{i}^{-}, e_{i}^{+}\right] \subset \mathbb{R}^{\mu}
$$

with

$$
\begin{aligned}
e_{i}^{+} & =(0, \ldots, 0,1,0, \ldots, 0), e_{i}^{-}=(0, \ldots, 0,-1,0, \ldots, 0) \in \mathbb{R}^{\mu}, \\
{\left[e_{i}^{-}, e_{i}^{+}\right] } & =\{(0, \ldots, 0, t, 0, \ldots, 0) \mid-1 \leqslant t \leqslant 1\} \subset \mathbb{R}^{\mu} .
\end{aligned}
$$

Thus $I_{\mu}$ is the one-point union of $\mu$ copies of the interval $[-1,1] \subset \mathbb{R}$, identifying the $\mu$ copies of $0 \in[-1,1]$.

We regard $B F_{\mu}$ as the quotient space of $I_{\mu}$

$$
B F_{\mu}=I_{\mu} /\left\{e_{i}^{+} \sim e_{i}^{-} \mid 1 \leqslant i \leqslant \mu\right\}=\bigvee_{\mu} S^{1},
$$

the one-point union of $\mu$ copies of the circle $S^{1}=[-1,1] /(-1 \sim 1)$ in which the $\mu$ copies of $[0] \in S^{1}$ are identified, with

$$
e_{i}=\left[e_{i}^{+}\right]=\left[e_{i}^{-}\right] \neq[0] \in B F_{\mu}
$$

a point in the $i^{\text {th }}$ circle. The universal cover $E F_{\mu}$ of $B F_{\mu}$ is

$$
E F_{\mu}=\left(F_{\mu} \times I_{\mu}\right) /\left\{\left(g, e_{i}^{+}\right) \sim\left(g z_{i}, e_{i}^{-}\right) \mid g \in F_{\mu}, 1 \leqslant i \leqslant \mu\right\},
$$

a contractible space with a free $F_{\mu}$-action

$$
F_{\mu} \times E F_{\mu} \rightarrow E F_{\mu} ;(g,(h, x)) \mapsto(g h, x)
$$

and covering projection

$$
p_{\mu}: E F_{\mu} \rightarrow B F_{\mu} ;[g, x] \mapsto[x] .
$$

Define an $F_{\mu}$-equivariant homeomorphism $G_{\mu} \stackrel{\cong}{\longrightarrow} E F_{\mu}$ by sending the vertex $g \in G_{\mu}^{(0)}=F_{\mu}$ to the point $(g, 0) \in E F_{\mu}$, and the edge $\left(g, g z_{i}\right) \in G_{\mu}^{(1)}$ to the line segment

$$
\left\{\left(g, t e_{i}^{+}\right) \mid 0 \leqslant t \leqslant 1\right\} \cup\left\{\left(g z_{i}, t e_{i}^{-}\right) \mid 0 \leqslant t \leqslant 1\right\} \subset E F_{\mu}
$$


with endpoints $(g, 0),\left(g z_{i}, 0\right) \in E F_{\mu}$. The projection $G_{\mu} \rightarrow G_{\mu} / F_{\mu}$ can thus be identified with the universal cover $p_{\mu}: E F_{\mu} \rightarrow B F_{\mu}$.

\subsection{Fundamental domains}

Definition 1.4 A fundamental domain of an $F_{\mu}$-cover $p: \widetilde{W} \rightarrow W$ is a closed subspace $U \subset \widetilde{W}$ such that

(a) $F_{\mu} U=\widetilde{W}$, or equivalently $p(U)=W$,

(b) for any $g, h \in F_{\mu}$

$$
g U \cap h U= \begin{cases}g V_{i} & \text { if } g=h z_{i} \\ h V_{i} & \text { if } g=h z_{i}^{-1} \\ g U & \text { if } g=h \\ \varnothing & \text { otherwise }\end{cases}
$$

with $V_{i}=U \cap z_{i}^{-1} U$.

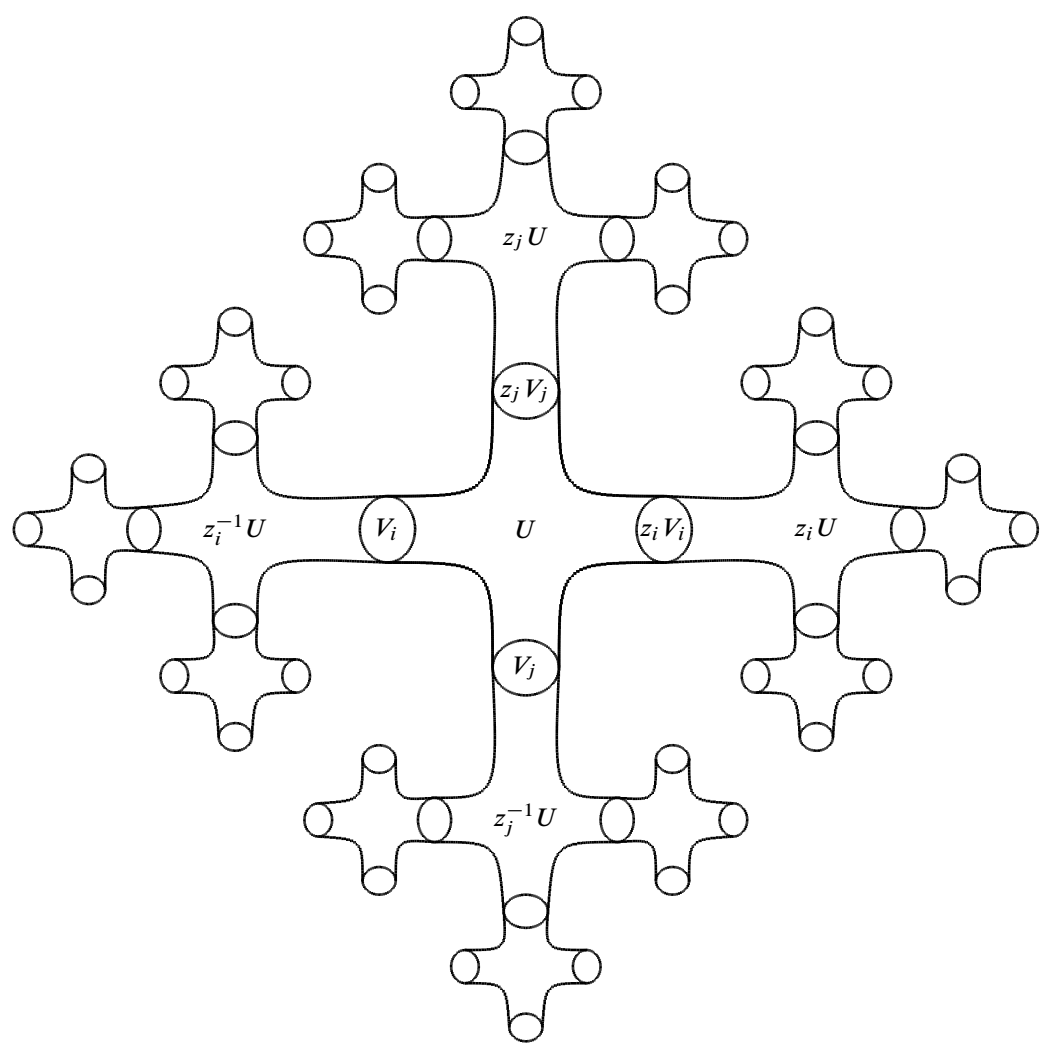


Thus $U \subset \widetilde{W}$ is sufficiently large for the translates $g U \subset \widetilde{W}\left(g \in F_{\mu}\right)$ to cover $\widetilde{W}$, but sufficiently small for the overlaps $g U \cap h U$ to be non-empty only if $g^{-1} h=1$ or $z_{i}$ or $z_{i}^{-1}$.

Example 1.5 (i) The subspace $\left(1, I_{\mu}\right) \subset E F_{\mu}$ is a fundamental domain of the universal cover $p_{\mu}: E F_{\mu} \rightarrow B F_{\mu}$.

(ii) Let $G_{\mu}^{\prime}$ be the barycentric subdivision of the Cayley tree $G_{\mu}$, the tree with

$$
\begin{aligned}
& \left(G_{\mu}^{\prime}\right)^{(0)}=G_{\mu}^{(0)} \cup G_{\mu}^{(1)}, \\
& \left(G_{\mu}^{\prime}\right)^{(1)}=\left\{\left(h,\left(g, g z_{i}\right)\right) \mid h=g \text { or } g z_{i}\right\} \subset\left(G_{\mu}^{\prime}\right)^{(0)} \times\left(G_{\mu}^{\prime}\right)^{(0)} .
\end{aligned}
$$

The $F_{\mu}$-equivariant homeomorphism $G_{\mu}=G_{\mu}^{\prime} \cong E F_{\mu}$ sends the vertex $\left(g, g z_{i}\right) \in$ $\left(G_{\mu}^{\prime}\right)^{(0)}$ to $\left(g, e_{i}^{+}\right) \in E F_{\mu}$. The subgraph $U_{\mu} \subset G_{\mu}^{\prime}$ defined by

$$
\begin{aligned}
& U_{\mu}^{(0)}=\{1\} \cup\left\{\left(1, z_{i}\right)\right\} \cup\left\{\left(z_{i}^{-1}, 1\right)\right\} \\
& U_{\mu}^{(1)}=\left\{\left(1,\left(1, z_{i}\right)\right)\right\} \cup\left\{\left(z_{i}^{-1},\left(z_{i}^{-1}, 1\right)\right)\right\}
\end{aligned}
$$

is the fundamental domain of the cover $G_{\mu} \rightarrow G_{\mu} / F_{\mu}$ corresponding to $\left(1, I_{\mu}\right) \subset E F_{\mu}$ under the $G_{\mu}$-equivariant homeomorphism $G_{\mu} \cong E F_{\mu}$.

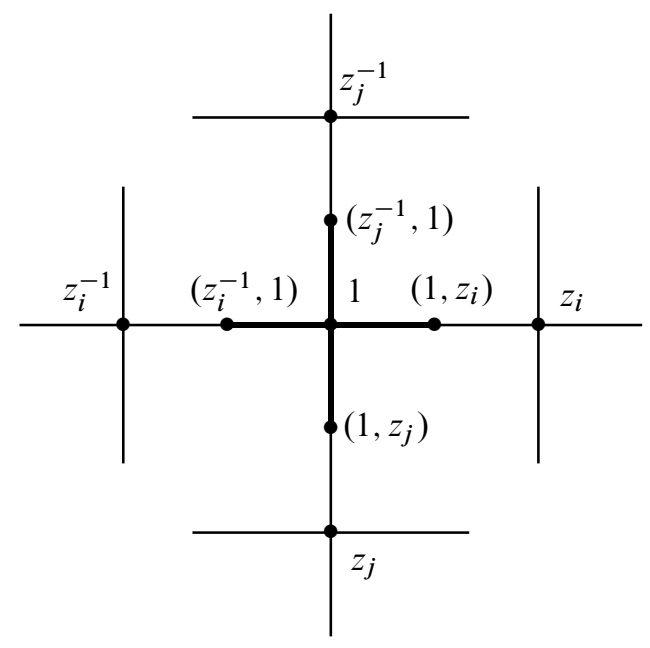

Proposition 1.6 (i) Given an $F_{\mu}$-cover $p: \widetilde{W} \rightarrow W$ and a map $f: V \rightarrow W$ let $f^{*} p: \widetilde{V}=f^{*} \widetilde{W} \rightarrow V$ be the pullback $F_{\mu}$-cover. If $U \subset \widetilde{W}$ is a fundamental domain of $p$ then

$$
\tilde{f}^{-1}(U)=\{(x, y) \mid x \in V, y \in U, f(x)=p(y) \in W\} \subset \tilde{V}
$$


is a fundamental domain of $f^{*} p$.

(ii) Every $F_{\mu}$-cover $p: \widetilde{W} \rightarrow W$ has fundamental domains.

Proof (i) By construction.

(ii) Apply (i), using the fundamental domain $U_{\mu} \subset G_{\mu}^{\prime}=E F_{\mu}$ for the cover

$$
p_{\mu}: E F_{\mu} \rightarrow E F_{\mu} / F_{\mu}=B F_{\mu}
$$

given by Example 1.5, noting that

$$
p=c^{*} p_{\mu}: \widetilde{W}=c^{*} E F_{\mu} \rightarrow W
$$

is the pullback of the universal $F_{\mu}$-cover $p_{\mu}: E F_{\mu} \rightarrow B F_{\mu}$ along a classifying map $c: W \rightarrow B F_{\mu}$

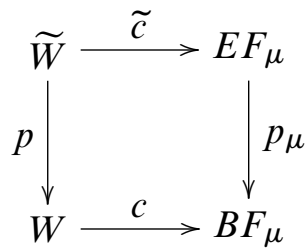

The inverse image of $U_{\mu} \subset E F_{\mu}$

$$
U=\widetilde{c}^{-1}\left(U_{\mu}\right) \subset \widetilde{W}
$$

is a fundamental domain of $c: \widetilde{W} \rightarrow W$.

\subsection{Combinatorial transversality}

If $p: \widetilde{W} \rightarrow W$ is an $F_{\mu}$-cover of a space $W$ with an additional structure such as a manifold or finite $C W$ complex, we should like to have fundamental domains $U \subset \widetilde{W}$ with the additional structure. For manifolds this is achieved by choosing a classifying map $c: W \rightarrow B F_{\mu}$ transverse at $\left\{e_{1}, e_{2}, \ldots, e_{\mu}\right\} \subset B F_{\mu}$ - see Example 1.11 below for a more detailed discussion. For a finite $C W$ complex $W$ we shall develop a combinatorial version of transversality, constructing finite subcomplexes $X \subset X(\infty)$ of the Borel construction $X(\infty)=\widetilde{W} \times{ }_{F_{\mu}} G_{\mu}$, such that the projection $f(\infty): W(\infty) \rightarrow W$ restricts to a simple homotopy equivalence $f: X \rightarrow W$ such that the pullback $F_{\mu}$-cover $\widetilde{X}=f^{*} \widetilde{W} \rightarrow X$ has a fundamental domain $U \subset \widetilde{X}$ which is a finite subcomplex.

Proposition 1.7 For any $F_{\mu}$-cover $p: \widetilde{W} \rightarrow W$ let $F_{\mu}$ act diagonally on $\widetilde{W} \times G_{\mu}$

$$
F_{\mu} \times\left(\widetilde{W} \times G_{\mu}\right) \rightarrow\left(\widetilde{W} \times G_{\mu}\right) ;(g,(x, y)) \mapsto(g x, g y) .
$$


(i) The map

$$
\pi: X=\widetilde{W} \times{ }_{F_{\mu}} G_{\mu} \rightarrow W ;[x, g] \mapsto p(x)
$$

is the projection of a fibration

$$
G_{\mu} \longrightarrow X \stackrel{\pi}{\longrightarrow} W
$$

with contractible point inverses; for each $x \in \widetilde{W}$ there is defined a homeomorphism

$$
G_{\mu} \rightarrow \pi^{-1} p(x) ; g \mapsto[x, g] .
$$

In particular, $\pi$ is a homotopy equivalence.

(ii) The pullback $F_{\mu}$-cover of $X$

$$
\pi^{*} p: \widetilde{X}=p^{*} \widetilde{W}=\widetilde{W} \times G_{\mu} \rightarrow X=\widetilde{W} \times{ }_{F_{\mu}} G_{\mu}
$$

has fundamental domain $\widetilde{W} \times U \subset \widetilde{X}=\widetilde{W} \times G_{\mu}$, with $U \subset G_{\mu}$ any fundamental domain.

Proof Standard.

Definition 1.8 (i) An $F_{\mu}$-splitting $(X, Y, Z, h)$ of a space $W$ is a homeomorphism $h: X \rightarrow W$ from a space with a decomposition

$$
X=Y \times[-1,1] \cup_{Y \times\{-1,1\}} Z
$$

with $Y=Y_{1} \sqcup Y_{2} \sqcup \ldots \sqcup Y_{\mu}$ the disjoint union of spaces $Y_{1}, Y_{2}, \ldots, Y_{\mu}$ and $Y \times[-1,1]$ attached to $Z$ along maps

$$
\alpha_{i}^{-}: Y_{i} \times\{-1\} \rightarrow Z, \alpha_{i}^{+}: Y_{i} \times\{1\} \rightarrow Z
$$

(ii) An $F_{\mu}$-splitting $(X, Y, Z, h)$ of a connected space $W$ is connected if each of $Y_{1}, Y_{2}, \ldots, Y_{\mu}, Z$ is non-empty and connected.

Proposition 1.9 Let $W$ be a space with an $F_{\mu}$-splitting $(X, Y, Z, h)$.

(i) The $F_{\mu}$-splitting determines an $F_{\mu}$-cover $p: \widetilde{W} \rightarrow W$ with

$$
\begin{aligned}
\widetilde{W}=\left(F_{\mu} \times\right. & (Y \times[-1,1] \sqcup Z)) / \sim, \\
& \left(g, y_{i}, 1\right) \sim\left(z_{i} g, \alpha_{i}^{+}\left(y_{i}, 1\right)\right), \\
& \left(g, y_{i},-1\right) \sim\left(g, \alpha_{i}^{-}\left(y_{i},-1\right)\right) \quad\left(g \in F_{\mu}, y_{i} \in Y_{i}, 1 \leqslant i \leqslant \mu\right),
\end{aligned}
$$

$p: \widetilde{W} \rightarrow W ;(g, x) \mapsto[h(x)]$. 
The subspace

$$
Z^{\prime}=(1, Y \times[0,1]) \cup(1, Z) \cup \bigcup_{i=1}^{\mu}\left(z_{i}, Y_{i} \times[-1,0]\right) \subset \widetilde{W}
$$

is a fundamental domain of $p: \widetilde{W} \rightarrow W$.

(ii) If there exists a homeomorphism $\phi: Z^{\prime} \rightarrow Z$ such that

$$
\phi\left(1, y_{i}, 0\right)=\alpha_{i}^{-}\left(y_{i},-1\right), \phi\left(z_{i}, y_{i}, 0\right)=\alpha_{i}^{+}\left(y_{i}, 1\right) \quad\left(y_{i} \in Y_{i}, 1 \leqslant i \leqslant \mu\right)
$$

the identification space

$$
\widetilde{W}^{\prime}=\left(F_{\mu} \times Z\right) /\left(g, \alpha_{i}^{-}\left(y_{i}\right)\right) \sim\left(z_{i} g, \alpha_{i}^{+}\left(y_{i}\right)\right)
$$

is such that there is defined a homeomorphism

$$
(1, \phi): \widetilde{W} \rightarrow \widetilde{W}^{\prime} ;(g, x) \mapsto(g, \phi(x))
$$

so that

$$
p^{\prime}=p(1, \phi)^{-1}: \widetilde{W}^{\prime} \rightarrow W ;(g, x) \mapsto p \phi^{-1}(x)
$$

is an $F_{\mu}$-cover of $W$ which is isomorphic to $p: \widetilde{W} \rightarrow W$, with fundamental domain

$$
(1, \phi)\left(Z^{\prime}\right)=(1, Z) \subset \widetilde{W}^{\prime} .
$$

(iii) The fundamental group of a connected space $W$ with a connected $F_{\mu}$-splitting $(X, Y, Z, h)$ is an amalgamated free product

$$
\pi_{1}(W)=\pi_{1}(Z) * F_{\mu} /\left\{\alpha_{i}^{+}\left(g_{i}\right) z_{i}=z_{i} \alpha_{i}^{-}\left(g_{i}\right) \mid g_{i} \in \pi_{1}\left(Y_{i}\right), 1 \leqslant i \leqslant \mu\right\} .
$$

The surjection $\pi_{1}(W) \rightarrow F_{\mu}$ is induced by a map $c: W \rightarrow B F_{\mu}$ sending $h\left(Y_{i} \times\{0\}\right) \subset$ $W$ to $\left\{e_{i}\right\} \subset B F_{\mu}$. The surjection $\pi_{1}(W) \rightarrow F_{\mu}$ classifies the connected $F_{\mu}$-cover $p: \widetilde{W} \rightarrow W$ in (i).

Proof (i) and (ii) follow by construction.

(iii) follows from the Seifert-van Kampen theorem and obstruction theory.

Example 1.10 Define an $F_{\mu}$-splitting $\left(H_{\mu},\{1,2, \ldots, \mu\}, I_{\mu}, f\right)$ of $B F_{\mu}$ by

$$
\begin{aligned}
& H_{\mu}=\{1,2, \ldots, \mu\} \times[-1,1] \cup_{(i, 1) \backsim e_{i}^{+},(i,-1) \backsim e_{i}^{-}} I_{\mu}, \\
& f: H_{\mu} \rightarrow B F_{\mu} ; \begin{cases}(i, t) \mapsto\left[(1-t / 2) e_{i}^{+}\right] & \text {for } 0 \leqslant t \leqslant 1 \\
(i, t) \mapsto\left[(1+t / 2) e_{i}^{-}\right] & \text {for }-1 \leqslant t \leqslant 0 \\
u \mapsto u / 2 & \text { for } u \in I_{\mu}\end{cases}
\end{aligned}
$$


with

$$
f(i, 0)=e_{i}, f(i, 1)=e_{i}^{+} / 2, f(i,-1)=e_{i}^{-} / 2 .
$$

The corresponding $F_{\mu}$-cover of $B F_{\mu}$ is the universal $F_{\mu}$-cover $\widetilde{B F} \mu=G_{\mu} \rightarrow B F_{\mu}$, with fundamental domain $I_{\mu}=\left(1, I_{\mu}\right) \subset G_{\mu}$. Note that $f\left(I_{\mu}\right)=J_{\mu}$, with $J_{\mu} \subset I_{\mu}$ the homeomorphic copy of $I_{\mu}$ defined by

$$
J_{\mu}=\left\{(0, \ldots, 0, t, 0, \ldots, 0) \in I_{\mu} \mid-1 / 2 \leqslant t \leqslant 1 / 2\right\} .
$$

A subspace $Y \subset X$ is collared if the inclusion $i: Y \rightarrow X$ extends to an embedding $j: Y \times[0,1] \rightarrow X$, with $i(y)=j(y, 0) \in X$ for $y \in Y$. In particular, $\partial Z \subset Z$ is collared, for any manifold with boundary $(Z, \partial Z)$.

Example 1.11 Use the $F_{\mu}$-splitting $\left(H_{\mu},\{1,2, \ldots, \mu\}, I_{\mu}, f\right)$ of $B F_{\mu}$ given by Example 1.10 to identify

$$
B F_{\mu}=H_{\mu}=\{1,2, \ldots, \mu\} \times[-1,1] \cup_{\{1,2, \ldots, \mu\} \times\{-1,1\}} I_{\mu} .
$$

If $p: \widetilde{X} \rightarrow X$ is an $F_{\mu}$-cover of a manifold $X$ it is possible to choose a classifying map

$$
c: X \rightarrow B F_{\mu}=\{1,2, \ldots, \mu\} \times[-1,1] \cup_{\{1,2, \ldots, \mu\} \times\{-1,1\}} I_{\mu}
$$

which is transverse regular at $\left\{e_{1}, e_{2}, \ldots, e_{\mu}\right\} \subset B F_{\mu}$, with the inverse images of $e_{i}=(i, 0) \in B F_{\mu}$ disjoint framed codimension-1 submanifolds

$$
Y_{i}=c^{-1}\left(e_{i}\right) \subset X \quad(1 \leqslant i \leqslant \mu) .
$$

Cutting $X$ along

$$
Y=c^{-1}\left\{e_{1}, e_{2}, \ldots, e_{\mu}\right\}=Y_{1} \sqcup Y_{2} \sqcup \ldots \sqcup Y_{\mu} \subset X
$$

there is obtained an $F_{\mu}$-splitting $(X, Y, Z$, id.) of $X$, so that

$$
X=Y \times[-1,1] \cup_{Y \times\{-1,1\}} Z
$$

with $Y=Y \times\{0\} \subset X$ a framed codimension-1 submanifold, and $Z=c^{-1}\left(I_{\mu}\right) \subset X$ a codimension -0 submanifold with

$$
\alpha_{i}^{+}: Y_{i} \times\{1\} \rightarrow Z, \quad \alpha_{i}^{-}: Y_{i} \times\{-1\} \rightarrow Z
$$

components of the inclusion of the boundary $\partial Z=Y \times\{-1,1\} \subset Z$. Since $\partial Z \subset Z$ is collared the fundamental domain of the $F_{\mu}$-cover $\widetilde{X}=c^{*} G_{\mu}$

$$
Z^{\prime}=(1, Y \times[0,1]) \cup(1, Z) \cup \bigcup_{i=1}^{\mu}\left(z_{i}, Y_{i} \times[-1,0]\right) \subset \widetilde{X}
$$


is such that there exists a homeomorphism $\phi: Z^{\prime} \rightarrow Z$ with

$$
\phi\left(1, y_{i}, 0\right)=\alpha_{i}^{-}\left(y_{i},-1\right), \phi\left(z_{i}, y_{i}, 0\right)=\alpha_{i}^{+}\left(y_{i}, 1\right) \quad\left(y_{i} \in Y_{i}, 1 \leqslant i \leqslant \mu\right) .
$$

Thus by Proposition 1.9 (ii) $p: \widetilde{X} \rightarrow X$ is isomorphic to the $F_{\mu}$-cover $p^{\prime}: \widetilde{X}^{\prime} \rightarrow X$ with

$$
\begin{aligned}
& \widetilde{X}^{\prime}=\left(F_{\mu} \times Z\right) /\left(g, \alpha_{i}^{-}\left(y_{i}\right)\right) \sim\left(z_{i} g, \alpha_{i}^{+}\left(y_{i}\right)\right), \\
& p^{\prime}=p(1, \phi)^{-1}: \widetilde{X}^{\prime} \rightarrow X ;(g, x) \mapsto p \phi^{-1}(x) .
\end{aligned}
$$

If $X$ and $\widetilde{X}$ are connected it is possible to choose $c$ such that each $Y_{i}=p^{-1}\left(e_{i}\right)$ is connected, with

$$
p_{*}=p(Y, Z)_{*}: \pi_{1}(X) \rightarrow F_{\mu} .
$$

Definition 1.12 (i) A homotopy $F_{\mu}$-splitting $(X, Y, Z, h)$ of a space $W$ is a homotopy equivalence $h: X \rightarrow W$ from a space with an $F_{\mu}$-splitting $(X, Y, Z, 1)$, so that

$$
X=Y \times[-1,1] \cup_{Y \times\{-1,1\}} Z, Y=Y_{1} \sqcup Y_{2} \sqcup \ldots \sqcup Y_{\mu} .
$$

(ii) A homotopy $F_{\mu}$-splitting $(X, Y, Z, h)$ of a finite $C W$ complex $W$ is simple if $X$ is a finite $C W$ complex, $Y_{1}, Y_{2}, \ldots, Y_{\mu}, Z \subset X$ are subcomplexes and $h: W \rightarrow X$ is a simple homotopy equivalence.

Example 1.13 Any finite $C W$ complex $W$ with an $F_{\mu}$-cover $\widetilde{W} \rightarrow W$ admits simple homotopy $F_{\mu}$-splittings $(X, Y, Z, h)$ : embed $W \subset S^{N}$ ( $N$ large) with closed regular neighbourhood $(X, \partial X)$ and apply the manifold transversality of Example 1.11 to the $F_{\mu}$-cover $\widetilde{X} \simeq \widetilde{W} \rightarrow W \simeq X$.

Working as in Ranicki [35] we shall now develop a combinatorial transversality construction of simple homotopy $F_{\mu}$-splittings of $W$ using finite subcomplexes of the Borel construction (Proposition 1.7) $\widetilde{W} \times{ }_{F_{\mu}} G_{\mu}$, as follows.

Definition 1.14 The canonical homotopy $F_{\mu}$-splitting $(X(\infty), Y(\infty), Z(\infty), h(\infty))$ of a space $W$ with an $F_{\mu}$-cover $p: \widetilde{W} \rightarrow W$ is given by

$$
X(\infty)=Y(\infty) \times[-1,1] \cup_{Y(\infty) \times\{-1,1\}} Z(\infty)
$$

with

$$
\begin{aligned}
& \alpha(\infty)_{i}^{+}: Y(\infty)_{i}=\widetilde{W} \rightarrow Z(\infty)=\widetilde{W} \times I_{\mu} ; x \mapsto\left(z_{i} x, e_{i}^{+}\right), \\
& \alpha(\infty)_{i}^{-}: Y(\infty)_{i}=\widetilde{W} \rightarrow Z(\infty)=\widetilde{W} \times I_{\mu} ; x \mapsto\left(x, e_{i}^{-}\right), \\
& h(\infty): X(\infty) \rightarrow W ;(x, y) \mapsto p(x) .
\end{aligned}
$$


The map $h(\infty)$ is a homotopy equivalence since it is the composite

$$
h(\infty)=\pi \circ f: X(\infty) \stackrel{f}{\longrightarrow} \widetilde{W} \times_{F_{\mu}} G_{\mu} \stackrel{\pi}{\longrightarrow} W
$$

of the homeomorphism

$$
f: X(\infty) \rightarrow \widetilde{W} \times F_{\mu} G_{\mu} ; \begin{cases}(x, i, t) \mapsto\left(x,(1-t / 2) e_{i}^{+}\right) & \text {for } 0 \leqslant t \leqslant 1 \\ (x, i, t) \mapsto\left(z_{i} x,(1+t / 2) e_{i}^{-}\right) & \text {for }-1 \leqslant t \leqslant 0 \\ (x, u) \mapsto(x, u / 2) & \text { for } u \in I_{\mu}\end{cases}
$$

and the homotopy equivalence

$$
\pi: X=\widetilde{W} \times_{F_{\mu}} G_{\mu} \rightarrow W
$$

given by Proposition 1.7. For every $y \in G_{\mu}$ there is a unique $g \in F_{\mu}$ such that $g y \in I_{\mu} \backslash\left\{e_{1}^{+}, e_{2}^{+}, \ldots, e_{\mu}^{+}\right\}$, so that either $g y=t e_{i}^{+}$with $0 \leqslant t<1$, or $g y=t e_{i}^{-}$ with $0 \leqslant t \leqslant 1$, and

$$
\begin{array}{ll}
f^{-1}: \widetilde{W} \times_{F_{\mu}} G_{\mu} \rightarrow X(\infty): & \\
{[x, y] \mapsto \begin{cases}(g x, i, 2(1-t)) & \text { if } g y=t e_{i}^{+} \text {with } 1 / 2 \leqslant t<1 \\
\left(z_{i}^{-1} g x, i, 2(t-1)\right) & \text { if } g y=t e_{i}^{-} \text {with } 1 / 2 \leqslant t \leqslant 1 \\
(g x, g y) & \text { if } \left.2 g y \in I_{\mu} \text { (ie if }-1 / 2 \leqslant t \leqslant 1 / 2\right) .\end{cases} }
\end{array}
$$

Proposition 1.15 Given a space $W$ with $F_{\mu}$-cover $p: \widetilde{W} \rightarrow W$ and a subspace $V \subseteq \widetilde{W}$ let

$$
X(V)=Y(V) \times[-1,1] \cup_{Y(V) \times\{-1,1\}} Z(V) \subseteq X(\infty)
$$

with

$$
\begin{aligned}
& \alpha(V)_{i}^{+}: Y(V)_{i}=V \cap z_{i}^{-1} V \rightarrow Z(V)=V \times I_{\mu} ; x \mapsto\left(z_{i} x, e_{i}^{+}\right), \\
& \alpha(V)_{i}^{-}: Y(V)_{i}=V \cap z_{i}^{-1} V \rightarrow Z(V)=V \times I_{\mu} ; x \mapsto\left(x, e_{i}^{-}\right),
\end{aligned}
$$

and set

$$
h(V)=h(\infty) \mid: X(V) \rightarrow W ;(x, t) \mapsto p(x) .
$$

(i) For any $x \in V$

$$
\begin{aligned}
h(V)^{-1}(p(x)) & =\left\{(x, y) \in \widetilde{W} \times F_{\mu} G_{\mu} \mid y \in G_{\mu}(V, x)\right\} \\
& =\{x\} \times G_{\mu}(V, x) \subseteq X(V) \subseteq X(\infty)=\widetilde{W} \times_{F_{\mu}} G_{\mu}
\end{aligned}
$$


with $G_{\mu}(V, x) \subseteq G_{\mu}$ the subgraph defined by

$$
\begin{aligned}
& G_{\mu}(V, x)^{(0)}=\left\{g \in F_{\mu} \mid g x \in V\right\} \subseteq G_{\mu}^{(0)}=F_{\mu}, \\
& G_{\mu}(V, x)^{(1)}=\left\{(i, g) \mid g x, g z_{i} x \in V\right\} \subseteq G_{\mu}^{(1)}=\{1,2, \ldots, \mu\} \times F_{\mu} .
\end{aligned}
$$

(ii) The image of $h(V)$ is

$$
h(V)(X(V))=p(V) \subseteq W,
$$

so that $h(V)$ is surjective if and only if $p(V)=W$, if and only if $\bigcup_{g \in F_{\mu}} g V=\widetilde{W}$.

Proof By construction.

In particular, if $V=\widetilde{W}$ then

$$
(X(V), Y(V), Z(V), h(V))=(X(\infty), Y(\infty), Z(\infty), h(\infty))
$$

and $h(V): X(V)=X(\infty) \rightarrow W$ is a homotopy equivalence (since it has contractible point inverses).

Theorem 1.16 (Combinatorial transversality) Let $W$ be a connected finite $C W$ complex with a connected $F_{\mu}$-cover $p: \widetilde{W} \rightarrow W$. The canonical homotopy $F_{\mu}-$ splitting $(X(\infty), Y(\infty), Z(\infty), h(\infty))$ of $W$ is a union

$$
(X(\infty), Y(\infty), Z(\infty), h(\infty))=\bigcup_{\{V\}}(X(V), Y(V), Z(V), h(V))
$$

of simple homotopy $F_{\mu}$-splittings $(X(V), Y(V), Z(V), h(V))$ of $W$, with $\{V\}$ a collection of finite subcomplexes $V \subset \widetilde{W}$ such that

$$
p(V)=W, \quad \bigcup_{\{V\}} V=\widetilde{W} .
$$

In particular, there exist simple homotopy $F_{\mu}$-splittings of $W$.

Proof Let

$$
W=\bigcup D^{0} \cup \bigcup D^{1} \cup \ldots \cup \bigcup D^{n}
$$

be the given cell structure of $W$, with skeleta

$$
W^{(r)}=\bigcup D^{0} \cup \bigcup D^{1} \cup \ldots \cup \bigcup D^{r} .
$$

The characteristic maps $D^{r} \rightarrow W$ of the $r$-cells restrict to embeddings $D^{r} \backslash S^{r-1} \subset W$ on the interiors, and as a set $W$ is the disjoint union of the interiors

$$
W=\bigsqcup D^{0} \sqcup \bigsqcup\left(D^{1} \backslash S^{0}\right) \sqcup \ldots \sqcup \bigsqcup\left(D^{n} \backslash S^{n-1}\right) .
$$


Choose a lift of each $r$-cell $D^{r}$ in $W$ to an $r$-cell $\widetilde{D}^{r}$ in $\widetilde{W}$, so that

$$
\widetilde{W}=\bigcup_{g \in F_{\mu}} \bigcup g \widetilde{D}^{0} \cup \bigcup_{g \in F_{\mu}} \bigcup g \widetilde{D}^{1} \cup \ldots \cup \bigcup_{g \in F_{\mu}} \bigcup g \widetilde{D}^{n}
$$

Write $\phi: S^{r} \rightarrow W^{(r)}$ for the attaching maps of the $(r+1)$-cells in $W$, and let $\widetilde{\phi}: S^{r} \rightarrow$ $\widetilde{W}^{(r)}$ be the attaching maps of the chosen lifted $(r+1)$-cells in $\widetilde{W}$. For any subtree $T_{n} \subseteq G_{\mu}$ there exists a sequence of subtrees $T_{r} \subseteq G_{\mu}$ for $r=n-1, n-2, \ldots, 0$ such that

$$
\widetilde{\phi}\left(S^{r}\right) \subseteq \widetilde{W}^{(r-1)} \cup \bigcup_{g_{r} \in T_{r}^{(0)}} g_{r} \widetilde{D}^{r} .
$$

The sequence $T=\left(T_{n}, T_{n-1}, \ldots, T_{0}\right)$ determines a subcomplex

$$
V\langle T\rangle=\bigcup_{g_{0} \in T_{0}^{(0)}} \bigcup g_{0} \widetilde{D}^{0} \cup \bigcup_{g_{1} \in T_{1}^{(0)}} \bigcup g_{1} \widetilde{D}^{1} \cup \ldots \cup \underset{g_{n} \in T_{n}^{(0)}}{\bigcup} \bigcup g_{n} \widetilde{D}^{n} \subseteq \widetilde{W}
$$

such that $p(V\langle T\rangle)=W$. The map $h(V\langle T\rangle): X(V\langle T\rangle) \rightarrow W$ constructed in Proposition 1.15 is surjective, with contractible point inverses

$$
h(V\langle T\rangle)^{-1}(p(x))=G_{\mu}(V, x)=T_{r} \quad\left(p(x) \in D^{r} \backslash S^{r-1} \subset W\right),
$$

so that it is a homotopy equivalence and $(X(V\langle T\rangle), Y(V\langle T\rangle), Z(V\langle T\rangle), h(V\langle T\rangle))$ is a homotopy $F_{\mu}$-splitting of $W$. For the maximal sequence $T=\left(G_{\mu}, G_{\mu}, \ldots, G_{\mu}\right)$ $V\langle T\rangle=\widetilde{W}$ and we have the canonical homotopy $F_{\mu}$-splitting $(X(\infty), Y(\infty), Z(\infty)$, $h(\infty))$ of $W$. Any finite subtree $T_{n} \subset G_{\mu}$ can be used to start a sequence $T=$ $\left(T_{n}, T_{n-1}, \ldots, T_{0}\right)$ of finite subtrees $T_{r} \subset G_{\mu}$ satisfying $(*)$, since for each $r=$ $n, n-1, \ldots, 1$ the $r$-cells $\widetilde{D}^{r} \rightarrow \widetilde{W}$ are attached to a finite subcomplex of the $(r-1)$-skeleton $\widetilde{W}^{(r-1)}$. For a sequence $T$ of finite subtrees $(X(V\langle T\rangle), Y(V\langle T\rangle)$, $Z(V\langle T\rangle), h(V\langle T\rangle))$ is a simple homotopy $F_{\mu}$-splitting of $W$. Finally, note that $G_{\mu}$ is a union of finite subtrees $T_{n} \subset G_{\mu}$, so that $\left(F_{\mu}, F_{\mu}, \ldots, F_{\mu}\right)$ is a union of sequences $T=\left(T_{n}, T_{n-1}, \ldots, T_{0}\right)$ of finite subtrees $T_{r} \subset G_{\mu}$ satisfying $(*)$, with corresponding expressions

$$
\begin{gathered}
\widetilde{W}=\bigcup_{T} V\langle T\rangle, \\
(X(\infty), Y(\infty), Z(\infty), h(\infty))=\bigcup_{T}(X(V\langle T\rangle), Y(V\langle T\rangle), Z(V\langle T\rangle), h(V\langle T\rangle)) .
\end{gathered}
$$

This completes the proof. 


\section{Algebraic transversality for $A\left[F_{\mu}\right]$-module complexes}

Algebraic transversality for $A\left[F_{\mu}\right]$-module chain complexes is modelled on the combinatorial transversality for $F_{\mu}$-covers of Section 1 . The procedure replaces matrices with entries in $A\left[F_{\mu}\right]$ by (in general larger) matrices with entries of the linear type

$$
a_{1}+\sum_{i=1}^{\mu} a_{z_{i}} z_{i} \in A\left[F_{\mu}\right] \quad\left(a_{1}, a_{z_{1}}, \ldots, a_{z_{\mu}} \in A\right) .
$$

Algebraic transversality can be traced back to the work of Higman, Bass-Heller-Swan, Stallings, Casson and Waldhausen on the algebraic $K$-theory of polynomial extensions and more general amalgamated free products. See of Ranicki [33, Chapter 7] for a treatment of algebraic transversality in the case $\mu=1$ when $A\left[F_{\mu}\right]=A\left[z, z^{-1}\right]$ is the Laurent polynomial extension of $A$.

Definition 2.1 Given an $A$-module $P$ and a set $F$ let

$$
P[F]=\bigoplus_{x \in F} x P
$$

be the direct sum of copies $x P$ of $P$, consisting of the formal $A$-linear combinations $\sum_{x \in F} x a_{x}\left(a_{x} \in P\right)$ with $\left\{x \in F \mid a_{x} \neq 0\right\}$ finite.

In particular, if $F$ is a semigroup with 1 then $A[F]$ is a ring.

We shall be particularly concerned with the case of a free group $F=F_{\mu}$ or the free semigroup $F_{\mu}^{+}$on $\mu$ generators $z_{1}, z_{2}, \ldots, z_{\mu}$. Thus $F_{\mu}^{+} \subset F_{\mu}$ consists of all the products $z_{i_{1}}^{n_{1}} z_{i_{2}}^{n_{2}} \ldots z_{i_{k}}^{n_{k}}$ with $n_{1}, n_{2}, \ldots, n_{k} \geqslant 0$. The rings $A\left[F_{\mu}\right], A\left[F_{\mu}^{+}\right]$are free products

$$
\begin{aligned}
& A\left[F_{\mu}\right]=A\left[z_{1}, z_{1}^{-1}\right] *_{A} A\left[z_{2}, z_{2}^{-1}\right] *_{A} \ldots *_{A} A\left[z_{\mu}, z_{\mu}^{-1}\right], \\
& A\left[F_{\mu}^{+}\right]=A\left[z_{1}\right] *_{A} A\left[z_{2}\right] *_{A} \cdots *_{A} A\left[z_{\mu}\right] .
\end{aligned}
$$

For any ring morphism $k: A \rightarrow B$ induction and restriction define functors

$$
\begin{aligned}
& k_{!}:\{A \text {-modules }\} \rightarrow\{B \text {-modules }\} ; L \mapsto k_{!} L=B \otimes_{A} L, \\
& k^{!}:\{B \text {-modules }\} \rightarrow\{A \text {-modules }\} ; M \mapsto k^{!} M=M
\end{aligned}
$$

such that $k_{\text {! }}$ is left adjoint to $k^{!}$, with a natural isomorphism

$$
\operatorname{Hom}_{A}\left(L, k^{!} M\right) \rightarrow \operatorname{Hom}_{B}\left(k_{!} L, M\right) ; f \mapsto(b \otimes x \mapsto b f(x)) .
$$


Definition 2.2 An $A[F]$-module is induced if it is of the form

$$
P[F]=k_{!} P=A[F] \otimes_{A} P
$$

for an $A$-module $P$, with $k: A \rightarrow A[F]$ the inclusion.

Proposition 2.3 Let $P, Q$ be $A$-modules.

(i) There is defined a natural isomorphism of additive groups

$$
\operatorname{Hom}_{A}(P, Q[F]) \rightarrow \operatorname{Hom}_{A[F]}(P[F], Q[F]) ; f \mapsto\left(\sum_{y \in F} y g_{y} \mapsto \sum_{y \in F} y f\left(g_{y}\right)\right) .
$$

(ii) There is defined a natural injection of additive groups

$$
\operatorname{Hom}_{A}(P, Q)[F] \rightarrow \operatorname{Hom}_{A}(P, Q[F]) ; \sum_{x \in F} x f_{x} \mapsto\left(y \mapsto \sum_{x \in F} x f_{x}(y)\right) .
$$

(iii) If $P$ is a f.g. projective $A$-module the injection in (ii) is also a surjection, so that the composite with the isomorphism in (i) is a natural isomorphism allowing the identification

$$
\operatorname{Hom}_{A}(P, Q)[F]=\operatorname{Hom}_{A[F]}(P[F], Q[F]) .
$$

Proof (i) This is just the adjointness of $k_{!}$and $k^{!}$, with $k: A \rightarrow A[F]$ the inclusion.

(ii) Obvious.

(iii) It is sufficient to consider the case $P=A$.

Definition 2.4 Let $P$ be an $A$-module which is given as a $\mu$-fold direct sum

$$
P=P_{1} \oplus P_{2} \oplus \cdots \oplus P_{\mu}
$$

with idempotents $\pi_{i}: P \rightarrow P_{i} \rightarrow P$.

(i) Define the $A[F]$-module endomorphism

$$
\begin{aligned}
z=\sum_{i=1}^{\mu} \pi_{i} z_{i}=\left(\begin{array}{cccc}
z_{1} & 0 & \cdots & 0 \\
0 & z_{2} & \cdots & 0 \\
\vdots & \vdots & \ddots & \vdots \\
0 & 0 & \cdots & z_{\mu}
\end{array}\right): P[F] & =P_{1}[F] \oplus P_{2}[F] \oplus \cdots \oplus P_{\mu}[F] \\
& \longrightarrow P[F]=P_{1}[F] \oplus P_{2}[F] \oplus \cdots \oplus P_{\mu}[F]
\end{aligned}
$$


For $F=F_{\mu}$ this is an automorphism, with inverse

$$
\begin{aligned}
& z^{-1}=\sum_{i=1}^{\mu} \pi_{i} z_{i}^{-1}=\left(\begin{array}{cccc}
z_{1}^{-1} & 0 & \cdots & 0 \\
0 & z_{2}^{-1} & \cdots & 0 \\
\vdots & \vdots & \ddots & \vdots \\
0 & 0 & \cdots & z_{\mu}^{-1}
\end{array}\right): P\left[F_{\mu}\right]=P_{1}\left[F_{\mu}\right] \oplus P_{2}\left[F_{\mu}\right] \oplus \cdots \oplus P_{\mu}\left[F_{\mu}\right] \\
& \longrightarrow P\left[F_{\mu}\right]=P_{1}\left[F_{\mu}\right] \oplus P_{2}\left[F_{\mu}\right] \oplus \cdots \oplus P_{\mu}\left[F_{\mu}\right] .
\end{aligned}
$$

(ii) Given a collection of $A$-module morphisms

$$
e=\left\{e_{i} \in \operatorname{Hom}_{A}\left(P_{i}, Q\right) \mid 1 \leqslant i \leqslant \mu\right\}
$$

define the $A[F]$-module morphism

$$
e z=\sum_{i=1}^{\mu} e \pi_{i} z_{i}=\left(\begin{array}{llll}
e_{1} z_{1} & e_{2} z_{2} & \cdots & e_{\mu} z_{\mu}
\end{array}\right): P[F]=P_{1}[F] \oplus P_{2}[F] \oplus \cdots \oplus P_{\mu}[F] \rightarrow Q[F] .
$$

(iii) An $A[F]$-module morphism $f: P[F] \rightarrow Q[F]$ is linear if

$$
\begin{gathered}
f=f^{+} z-f^{-}=\left(f^{+, 1} z_{1}-f^{-, 1} \ldots f^{+, \mu} z_{\mu}-f^{-, \mu}\right): \\
P[F]=P_{1}[F] \oplus P_{2}[F] \oplus \cdots \oplus P_{\mu}[F] \rightarrow Q[F]
\end{gathered}
$$

for some $A$-module morphisms $f^{+, i}, f^{-, i}: P_{i} \rightarrow Q$.

Definition 2.5 (i) A Mayer-Vietoris presentation of an $A[F]$-module $E$ is an exact sequence of the type

$$
0 \longrightarrow \bigoplus_{i=1}^{\mu} C^{(i)}[F] \stackrel{f}{\longrightarrow} D[F] \longrightarrow E \longrightarrow 0
$$

with $C^{(i)}, D A$-modules and $f=f^{+} z-f^{-}$a linear $A[F]$-module morphism.

(ii) A Mayer-Vietoris presentation of an $A[F]$-module morphism $\phi: E \rightarrow E^{\prime}$ is a morphism of Mayer-Vietoris presentations

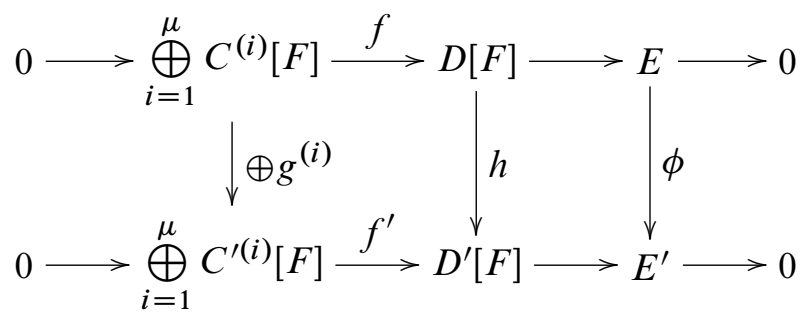


where $g^{(i)}: C^{(i)} \rightarrow C^{(i)}$ and $h: D \rightarrow D^{\prime} A$-module morphisms.

(iii) A Mayer-Vietoris presentation of an $A[F]$-module chain complex $E$ is an exact sequence as in (i), with $C^{(i)}, D A$-module chain complexes and $f^{ \pm, i}: C^{(i)} \rightarrow D$ $A$-module chain maps. Similarly for an $A[F]$-module chain map $\phi: E \rightarrow E^{\prime}$, with a morphism of exact sequences as in (ii) in which $g^{(i)}, h$ are $A$-module chain maps.

(iv) A Mayer-Vietoris presentation of a finite induced f.g. projective $A\left[F_{\mu}\right]$-module chain complex $E$ is finite if $C^{(i)}, D$ are finite f.g. projective $A$-module chain complexes.

Example 2.6 Let $X$ be the $C W$ complex

$$
X=Z /\left\{x \sim \beta_{i}(x) \mid x \in Y_{i}^{+}, 1 \leqslant i \leqslant \mu\right\}
$$

which is obtained from a $C W$ complex $Z$ and disjoint collared subcomplexes

$$
Y_{1}^{+}, Y_{2}^{+}, \ldots, Y_{\mu}^{+}, Y_{1}^{-}, Y_{2}^{-}, \ldots, Y_{\mu}^{-} \subset Z
$$

using cellular homeomorphisms $\beta_{i}: Y_{i}^{+} \rightarrow Y_{i}^{-}$as identifications. As in Definition 1.8 there is an $F_{\mu}$-splitting $(X, Y, Z, h)$, where $Y=Y_{1}^{+} \sqcup Y_{2}^{+} \sqcup \ldots \sqcup Y_{\mu}^{+}$and

$$
\begin{aligned}
& \alpha_{i}^{+}=\text {inclusion }_{Y_{i}^{+} \subset Z}: Y_{i}=Y_{i}^{+} \rightarrow Z, \\
& \left.\alpha_{i}^{-}=\operatorname{inclusion}_{Y_{i}^{-} \subset Z}\right) \beta_{i}: Y_{i}=Y_{i}^{+} \rightarrow Z .
\end{aligned}
$$

The cellular free $\mathbb{Z}\left[F_{\mu}\right]$-module chain complex $C(\widetilde{X})$ of the $F_{\mu}$-cover $\widetilde{X}$ of $X$ given by Proposition 1.9 (i) has a Mayer-Vietoris presentation

$$
0 \longrightarrow C(Y)\left[F_{\mu}\right] \stackrel{\alpha}{\longrightarrow} C(Z)\left[F_{\mu}\right] \longrightarrow C(\widetilde{X}) \longrightarrow 0
$$

with $C(Y)^{(i)}=C\left(Y_{i}\right), C(Z)$ free $\mathbb{Z}$-module chain complexes, and $\alpha=\alpha^{+} z-\alpha^{-}$a linear $\mathbb{Z}\left[F_{\mu}\right]$-module chain map. If $Z$ is a finite $C W$ complex the Mayer-Vietoris presentation is finite.

We shall construct Mayer-Vietoris presentations of free $A\left[F_{\mu}\right]-$ module chain complexes using the Cayley tree $G_{\mu}$ (Definition 1.3) and the subtree $G_{\mu}^{+} \subset G_{\mu}$ corresponding to $F_{\mu}^{+} \subset F_{\mu}$.

Definition 2.7 (i) Let $G_{\mu}^{+} \subset G_{\mu}$ be the subtree with

$$
\left(G_{\mu}^{+}\right)^{(0)}=F_{\mu}^{+}, \quad\left(G_{\mu}^{+}\right)^{(1)}=\left\{\left(g, g z_{i}\right) \mid g \in F_{\mu}^{+}, 1 \leqslant i \leqslant \mu\right\} .
$$


(ii) For any subtree $T \subseteq G_{\mu}$ and $i=1,2, \ldots, \mu$ let $T^{(i, 1)} \subseteq T^{(1)}$ be the set of edges of type $\left(g, g z_{i}\right)$ with $g \in F_{\mu}$, such that

and let

$$
T^{(1)}=\coprod_{i=1}^{\mu} T^{(i, 1)},
$$

$$
T^{+}=T \cap G_{\mu}^{+} \subseteq T .
$$

(iii) For $F=F_{\mu}\left(\right.$ resp. $\left.F_{\mu}^{+}\right)$let $G=G_{\mu}\left(\right.$ resp. $\left.G_{\mu}^{+}\right)$.

We shall only be considering subtrees $T \subseteq G$ containing the vertex $1 \in G^{(0)}$.

Proposition 2.8 Given an $A$-module $P$ let $E=P[F]$ be the induced $A[F]$-module, regarded as a $0-$ dimensional $A[F]$-module chain complex.

(i) For any subtree $T \subseteq G$ there is defined a Mayer-Vietoris presentation of $E$

$$
E\langle T\rangle: 0 \longrightarrow \bigoplus_{i=1}^{\mu} C^{(i)}[F] \stackrel{f}{\longrightarrow} D[F] \longrightarrow E \longrightarrow 0
$$

with

$$
\begin{aligned}
& D=P\left[T^{(0)}\right], \quad C^{(i)}=D \cap z_{i}^{-1} D=P\left[T^{(i, 1)}\right] \subseteq E, \\
& f^{+, i}: C^{(i)} \rightarrow D ; x p \mapsto x p, \quad f^{-, i}: C^{(i)} \rightarrow D ; x p \mapsto z_{i} x p .
\end{aligned}
$$

(ii) The Mayer-Vietoris presentations $E\langle T\rangle$ are such that

$$
E\left\langle T \cap T^{\prime}\right\rangle=E\langle T\rangle \cap E\left\langle T^{\prime}\right\rangle, \quad E\left\langle T \cup T^{\prime}\right\rangle=E\langle T\rangle+E\left\langle T^{\prime}\right\rangle \quad\left(T, T^{\prime} \subseteq G\right) .
$$

If $P$ is f.g. projective and $T$ is finite then $C^{(i)}, D$ are f.g. projective $A$-modules.

(iii) Given a morphism of induced $A[F]$-modules

$$
\phi: E=P[F] \rightarrow E^{\prime}=P^{\prime}[F]
$$

and a subtree $T \subseteq G$ let $\phi_{*} T \subseteq G$ be the smallest subtree such that

$$
\phi(P) \subseteq P^{\prime}\left[\phi_{*} T^{(0)}\right] \subseteq E^{\prime} .
$$

For any subtree $T^{\prime} \subseteq G$ such that $\phi_{*} T \subseteq T^{\prime}$ there is defined a morphism of MayerVietoris presentations

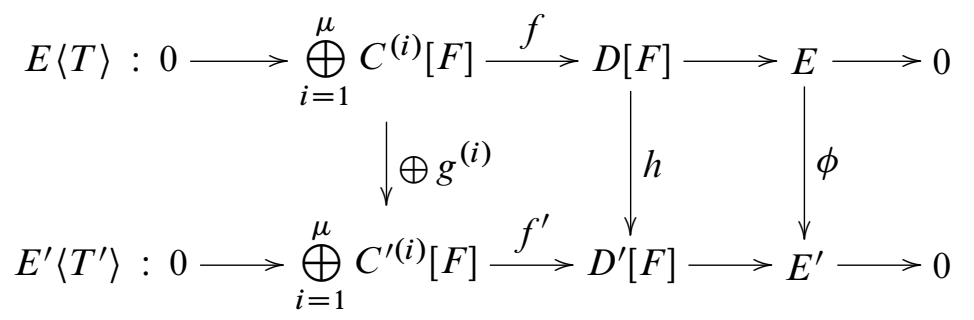


with

$$
g^{(i)}=\phi\left|: C^{(i)} \rightarrow C^{\prime(i)}, \quad h=\phi\right|: D \rightarrow D^{\prime} .
$$

If $P$ is a f.g. $A$-module and $T \subset G$ is finite, then so is $\phi_{*} T \subset G$.

Proof By construction.

Example 2.9 The Mayer-Vietoris presentation of $E$ associated to the minimal subtree $T=\{1\} \subset G$ is

$$
0 \longrightarrow 0 \longrightarrow P[F] \stackrel{\text { id. }}{\longrightarrow} E \longrightarrow 0 \text {. }
$$

Definition 2.10 The canonical Mayer-Vietoris presentation of an $A[F]$-module chain complex $E$ with each $E_{r}=P_{r}[F]$ an induced $A[F]$-module

$$
E\langle\infty\rangle: 0 \longrightarrow \bigoplus_{i=1}^{\mu} C^{(i)}[F] \stackrel{f}{\longrightarrow} D[F] \longrightarrow E \longrightarrow 0
$$

is the Mayer-Vietoris presentation with $E_{r}\langle\infty\rangle=E_{r}\langle T\rangle$ the Mayer-Vietoris presentation of $E_{r}$ associated to the maximal subtree $T=G \subseteq G$, where

$$
f^{+, i}=\text { id. }, f^{-}=z_{i}: C^{(i)}=k^{!} E \rightarrow D=k^{!} E
$$

with $k: A \rightarrow A[F]$ the inclusion.

Remark 2.11 (i) The canonical Mayer-Vietoris presentation can be written in terms of induction and restriction

$$
E\langle\infty\rangle: 0 \longrightarrow \bigoplus_{\mu} k_{!} k^{!} E \stackrel{f}{\longrightarrow} k_{!} k^{!} E \longrightarrow E \longrightarrow 0
$$

with

$$
\begin{aligned}
& f: \bigoplus_{\mu} k_{!} k^{!} E \rightarrow k_{!} k^{!} E ; x_{i} \otimes y \mapsto x_{i} z_{i} \otimes y-x_{i} \otimes z_{i} y \quad\left(x_{i} \in A[F], y \in E\right), \\
& k_{!} k^{!} E \rightarrow E ; x \otimes y \mapsto x y \quad(x \in A[F], y \in E) .
\end{aligned}
$$

(ii) The canonical Mayer-Vietoris presentation for $F=F_{\mu}$ is the algebraic analogue of the canonical homotopy $F_{\mu}$-splitting of a space $W$ with an $F_{\mu}$-cover $\widetilde{W}$ in Definition 1.14.

Theorem 2.12 (Algebraic transversality for chain complexes) Let $E$ be an $n-$ dimensional $A[F]$-module chain complex

$$
E: E_{n} \stackrel{d_{n}}{\longrightarrow} E_{n-1} \longrightarrow \cdots \longrightarrow E_{1} \stackrel{d_{1}}{\longrightarrow} E_{0}
$$


with each $E_{r}=P_{r}[F]$ induced from an $A$-module $P_{r}$.

(i) For any sequence $T=\left(T_{n}, T_{n-1}, \ldots, T_{0}\right)$ of subtrees $T_{r} \subseteq G$ such that

$$
\left(d_{r}\right)_{*}\left(T_{r}\right) \subseteq T_{r-1} \quad(r=n, n-1, \ldots, 1)
$$

there is defined a Mayer-Vietoris presentation

$$
E\langle T\rangle: 0 \longrightarrow \bigoplus_{i=1}^{\mu} C^{(i)}[F] \stackrel{f^{+}{ }^{-} f^{-}}{\longrightarrow} D[F] \longrightarrow 0
$$

with

$$
E\langle T\rangle_{r}=E_{r}\left\langle T_{r}\right\rangle \quad(0 \leqslant r \leqslant n), \quad E\langle T\rangle \subseteq E\langle\infty\rangle .
$$

(ii) If the $A$-modules $P_{r}$ are f.g. projective then for any finite subtree $T_{n} \subseteq G$ there exists a sequence $T=\left(T_{n}, T_{n-1}, \ldots, T_{0}\right)$ of finite subtrees $T_{r} \subseteq G$ satisfying (*), so that $E\langle T\rangle$ is a finite Mayer-Vietoris presentation of $E$. Thus

$$
E\langle\infty\rangle=\bigcup_{T} E\langle T\rangle
$$

with the union taken over all such sequences $T$. In particular, $E$ admits a finite Mayer-Vietoris presentation.

Proof By repeated applications of Proposition 2.8, with the sequences $T=\left(T_{n}, T_{n-1}\right.$, $\ldots, T_{0}$ ) the chain complex analogues of the sequences used to construct the homotopy $F_{\mu}$-splittings of $C W$ complexes in the proof of Theorem 1.16.

This completes the proof of Theorem A of the Introduction.

\section{Blanchfield and Seifert modules}

\subsection{The Magnus-Fox embedding}

This section obtains some technical results on the Magnus-Fox embedding which we shall need to characterize Blanchfield $A\left[F_{\mu}\right]$-modules, and to approximate h.d. 1 $F_{\mu}$-link modules by f.g. projective Seifert $A$-modules.

Let $A\left\langle\left\langle x_{1}, x_{2}, \ldots, x_{\mu}\right\rangle\right\rangle$ be the ring of $A$-coefficient formal power series in noncommuting indeterminates $x_{1}, x_{2}, \ldots, x_{\mu}$. The Magnus-Fox embedding is defined by

$$
i: A\left[F_{\mu}\right] \rightarrow \widehat{A\left[F_{\mu}\right]}=A\left\langle\left\langle x_{1}, x_{2}, \ldots, x_{\mu}\right\rangle\right\rangle ; z_{j} \mapsto 1+x_{j} .
$$


See the paper of Ara and Dicks [1] for a recent account of the Magnus-Fox embedding, including the relationship with noncommutative Cohn localization.

The augmentations $\epsilon\left(z_{j}\right)=1, \widehat{\epsilon}\left(x_{j}\right)=0$ give rise to a commutative triangle of rings

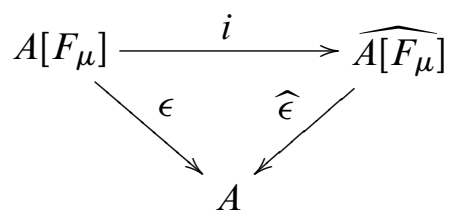

Proposition 3.1 (i) For projective $\widehat{A\left[F_{\mu}\right]}$-modules $\widehat{K}, \hat{L}$ the augmentation map

$$
\hat{\epsilon}: \operatorname{Hom}_{A\left[\widehat{F F}_{\mu}\right]}(\hat{K}, \hat{L}) \rightarrow \operatorname{Hom}_{A}\left(A \otimes_{A\left[\widehat{F}_{\mu}\right]} \hat{K}, A \otimes_{A\left[\widehat{F}_{\mu}\right]} \hat{L}\right) ; \hat{f} \mapsto 1 \otimes \widehat{f}
$$

is surjective.

(ii) A morphism $\hat{f}: \hat{K} \rightarrow \widehat{L}$ of projective $\widehat{A\left[F_{\mu}\right]}-$ modules is an isomorphism if and only if the $A$-module morphism

$$
1 \otimes \widehat{f}: A \otimes_{A\left[\widehat{F}_{\mu}\right]} \hat{K} \rightarrow A \otimes_{A\left[\widehat{F}_{\mu}\right]} \widehat{L}
$$

is an isomorphism.

(iii) A morphism $f: K \rightarrow L$ of projective $A\left[F_{\mu}\right]$-modules induces an $\widehat{A\left[F_{\mu}\right]}$-module isomorphism

$$
1 \otimes f: \widehat{A\left[F_{\mu}\right]} \otimes_{A\left[F_{\mu}\right]} K \rightarrow \widehat{A\left[F_{\mu}\right]} \otimes_{A\left[F_{\mu}\right]} L
$$

if and only if the $A$-module morphism

$$
1 \otimes f: A \otimes_{A\left[F_{\mu}\right]} K \rightarrow A \otimes_{A\left[F_{\mu}\right]} L
$$

is an isomorphism.

Proof (i) By additivity this reduces to the special case $\widehat{K}=\widehat{L}=\widehat{A\left[F_{\mu}\right]}$, which is just the fact that $\widehat{\epsilon}: \widehat{A\left[F_{\mu}\right]} \rightarrow A$ is surjective.

(ii) It suffices to prove that if $1 \otimes \widehat{f}$ is an $A$-module isomorphism then $\hat{f}$ is an $\widehat{A\left[F_{\mu}\right]}$-module isomorphism.

Consider first the special case when $\widehat{K}, \widehat{L}$ are free $\widehat{A\left[F_{\mu}\right]}$-modules, say $\widehat{A\left[F_{\mu}\right]^{k}}$, $\left.\widehat{A\left[F_{\mu}\right]}\right]^{\ell}$ for some sets $k, \ell$. The augmentation map

$$
\left.\widehat{\epsilon}: \operatorname{Hom}_{\widehat{A\left[F_{\mu}\right]}}\left(\widehat{A\left[F_{\mu}\right]^{k}}, \widehat{A\left[F_{\mu}\right.}\right]^{\ell}\right) \rightarrow \operatorname{Hom}_{A}\left(A^{k}, A^{\ell}\right) ; \hat{f} \mapsto 1 \otimes \widehat{f}
$$


has a canonical splitting. If $1 \otimes \widehat{f}$ is an isomorphism then all the entries in the matrix of the $\widehat{A\left[F_{\mu}\right]}$-module morphism

$$
\left.g=1-(1 \otimes \widehat{f})^{-1} \widehat{f}:{\widehat{A\left[F_{\mu}\right.}}^{k} \rightarrow \widehat{A\left[F_{\mu}\right.}\right]^{k}
$$

have constant term 0 , so that $1-g=(1 \otimes \widehat{f})^{-1} \widehat{f}$ is an $\widehat{A\left[F_{\mu}\right]}$-module isomorphism with inverse

$$
(1-g)^{-1}=1+g+g^{2}+g^{3}+g^{4}+\cdots:{\widehat{A\left[F_{\mu}\right.}}^{k} \rightarrow{\widehat{A\left[F_{\mu}\right]}}^{k},
$$

and $\widehat{f}=(1 \otimes \widehat{f})(1-g)$ is an isomorphism.

For the general projective case apply (i) to lift $(1 \otimes \widehat{f})^{-1}$ to an $\widehat{A\left[F_{\mu}\right]}-$ module morphism $\widehat{e}: \hat{L} \rightarrow \widehat{K}$. Choose a projective $\widehat{A\left[F_{\mu}\right]}$-module $\widehat{J}$ such that $\widehat{J} \oplus \hat{K} \oplus \hat{L}$ is a free $\widehat{A\left[F_{\mu}\right]}$-module, and apply the special case to the $\widehat{A\left[F_{\mu}\right]}$-module morphism

$$
1 \oplus\left(\begin{array}{ll}
0 & \widehat{e} \\
\hat{f} & 0
\end{array}\right): \widehat{J} \oplus \widehat{K} \oplus \widehat{L} \rightarrow \widehat{J} \oplus \widehat{K} \oplus \widehat{L}
$$

(iii) This is a special case of (ii).

For $j=1,2, \ldots, \mu$ let $y_{j}$ be a formal square root of $z_{j}$, so that $\left(y_{j}\right)^{2}=z_{j}$. Let $F_{\mu}(y)$ be the free group generated by $y_{1}, y_{2}, \ldots, y_{\mu}$, so that $F_{\mu} \subset F_{\mu}(y)$ is the free subgroup generated by $z_{1}, z_{2}, \ldots, z_{\mu}$. We can identify $G_{\mu}^{(1, j)}$ with the subset $F_{\mu} y_{j} \subset F_{\mu}(y)$ : the edge $\left(g, g z_{j}\right) \in G_{\mu}^{(1, j)}\left(g \in F_{\mu}\right)$ is identified with the element $g y_{j}^{-1} \in F_{\mu}(y)$.

Lemma 3.2 If $T \subset G_{\mu}$ is a finite subtree then

$$
A\left[T^{(0)}\right]=A[\{1\}] \oplus\left(\bigoplus_{j=1}^{\mu} A\left[T^{(1, j)}\right]\left(y_{j}^{-1}-y_{j}\right)\right) \subset A\left[F_{\mu}\right] .
$$

Proof If $w \in T^{(1, j)}$ then certainly $w\left(y_{j}^{-1}-y_{j}\right) \in A\left[T^{(0)}\right]$. Let us check linear independence of the generators on the right hand side of $(*)$. Assuming the contrary, let

$$
a_{1}+\sum_{g y_{j}^{-1} \in U} a_{g} g\left(y_{j}^{-1}-y_{j}\right)=0 \in A\left[F_{\mu}\right]
$$

be a non-trivial relation with $U \subset T^{(1)}$ non-empty and minimal. We reach a contradiction by observing that if $g\left(y_{j}\right)^{-1} \in U$ is a word of maximal length (in reduced form) then $a_{g}=0$. 
We must also show that every $v \in T^{(0)}$ is an element of the right-hand side of $(*)$. Indeed there is a (unique) path in the tree from 1 to $v$ defined by a sequence of edges $w_{1}, w_{2}, \ldots, w_{n} \in T^{(1)}$ and we have

$$
v=1+\sum_{i=1}^{n} w_{i}\left(y_{j(i)}^{-1}-y_{j(i)}\right) \eta_{i} \in A\left[F_{\mu}\right]
$$

if the signs $\eta_{i} \in\{ \pm 1\}$ are chosen appropriately and $j(i)$ is such that $w_{i} \in T^{(1, j(i))}$.

Proposition 3.3 For any finite subset $S \subset F_{\mu}$ the inclusion $\left.i \mid: A[S] \rightarrow \widehat{A\left[F_{\mu}\right.}\right]$ is a split $A$-module injection.

Proof Since every finite $S$ is contained in the vertex set of some finite tree we may assume that $S=T^{(0)}$ for some finite subtree $T \subset G_{\mu}$. We proceed by induction on $\left|T^{(0)}\right|$.

If the tree $T$ has only one vertex then $T^{(0)}=\{1\}$ with $\left.i(1)=1 \in \widehat{A\left[F_{\mu}\right.}\right]$ and

$$
\left.\widehat{A\left[F_{\mu}\right]}=A[\{1\}] \oplus \bigoplus_{i=1}^{\mu} \widehat{A\left[F_{\mu}\right]} x_{i}=A \oplus \bigoplus_{i=1}^{\mu} \widehat{A\left[F_{\mu}\right.}\right]\left(1-z_{i}^{\eta}\right)
$$

for any $\eta \in\{ \pm 1\}$, and $\left.i \mid: A[\{1\}] \rightarrow \widehat{A\left[F_{\mu}\right.}\right]$ is a split injection.

Suppose now that $\left|T^{(0)}\right| \geqslant 2$. Let $v_{0} \in T^{(0)}$ be a leaf, ie a vertex to which only one edge is incident. Let $T \backslash\left\{v_{0}\right\}$ denote the tree obtained by removing the vertex $v_{0}$ and the incident edge. By the inductive hypothesis, $i \mid: A\left[T^{(0)} \backslash\left\{v_{0}\right\}\right] \rightarrow \widehat{A\left[F_{\mu}\right]}$ is a split injection; we denote the image by $P$.

Since $v_{0}$ is incident to precisely one edge then $v_{0}=w_{0} y_{k}^{\eta}$ for unique $\eta \in\{ \pm 1\}$, $k \in\{1, \ldots, \mu\}$ and $w_{0} \in T^{(1, k)}$. Now for every $j$ we have $T^{(1, j)} y_{j}^{-\eta} \subset T^{(0)} \backslash\left\{v_{0}\right\}$. Thus

$$
\begin{aligned}
T^{(1, j)}\left(y_{j}^{-1}-y_{j}\right) & =T^{(1, j)} y_{j}^{-\eta}\left(1-y_{j}^{2 \eta}\right) \eta \\
& =T^{(1, j)} y_{j}^{-\eta}\left(1-z_{j}^{\eta}\right) \eta \subset\left(T^{(0)} \backslash\left\{v_{0}\right\}\right)\left(1-z_{j}^{\eta}\right) \eta .
\end{aligned}
$$

It follows from $(*)$ that $i\left(A\left[T^{(0)}\right]\right)$ is a direct summand of

$$
\operatorname{Ai}\left(v_{0}\right) \oplus \bigoplus_{j=1}^{\mu} P\left(1-z_{j}^{\eta}\right)
$$

and hence, by the following Lemma 3.4, a direct summand of $\widehat{A\left[F_{\mu}\right]}$. 
Lemma 3.4 Suppose $P$ is an $A$-module which is a direct summand of $\widehat{A\left[F_{\mu}\right]}$. If $\theta \in \widehat{A\left[F_{\mu}\right]}$ is an element such that $\widehat{\epsilon}(\theta)=1 \in A$ and $\eta=1$ or -1 then

$$
A \theta \oplus\left(\bigoplus_{j=1}^{\mu} P\left(1-z_{j}^{\eta}\right)\right) \subset \widehat{A\left[F_{\mu}\right]}
$$

is again a direct summand.

Proof We may write $\widehat{A\left[F_{\mu}\right]}=P \oplus Q$ for some $A$-module $Q$. Let $\eta=1$ or -1 . Now it follows easily from $(* *)$ that

$$
\begin{aligned}
\widehat{A\left[F_{\mu}\right]} & =A \theta \oplus\left(\bigoplus_{j=1}^{\mu} \widehat{A\left[F_{\mu}\right]}\left(1-z_{j}^{\eta}\right)\right) \\
& =A \theta \oplus\left(\bigoplus_{j=1}^{\mu} P\left(1-z_{j}^{\eta}\right)\right) \oplus\left(\bigoplus_{j=1}^{\mu} Q\left(1-z_{j}^{\eta}\right)\right)
\end{aligned}
$$

which completes the proof.

\subsection{Blanchfield modules}

Definition 3.5 (i) A Blanchfield $A\left[F_{\mu}\right]$-module $M$ is an $A\left[F_{\mu}\right]$-module such that

$$
\operatorname{Tor}_{*}^{A\left[F_{\mu}\right]}(A, M)=0 .
$$

(ii) (Sheiham [41]) An $F_{\mu}$-link module $M$ is an $A\left[F_{\mu}\right]$-module which has a 1dimensional induced $A\left[F_{\mu}\right]$-module resolution

$$
0 \longrightarrow P\left[F_{\mu}\right] \stackrel{d}{\longrightarrow} P\left[F_{\mu}\right] \longrightarrow M \longrightarrow 0
$$

with $P$ an $A$-module and $d$ an $A\left[F_{\mu}\right]$-module morphism such that the augmentation $A$-module morphism $\epsilon(d): P \rightarrow P$ is an isomorphism.

As before, let $k: A \rightarrow A\left[F_{\mu}\right]$ be the inclusion.

Proposition 3.6 The following conditions on an $A\left[F_{\mu}\right]$-module $M$ are equivalent:

(i) $M$ is a Blanchfield module,

(ii) $M$ is an $F_{\mu}$-link module, 
(iii) the $A$-module morphism

$$
\gamma_{M}: \bigoplus_{\mu} k^{!} M \rightarrow k^{!} M ;\left(m_{1}, m_{2}, \ldots, m_{\mu}\right) \mapsto \sum_{i=1}^{\mu}\left(z_{i}-1\right) m_{i}
$$

is an isomorphism.

Proof The canonical Mayer-Vietoris presentation (Definition 2.10) of any $A\left[F_{\mu}\right]-$ module $M$ is defined by

$$
0 \longrightarrow \bigoplus_{\mu} k_{!} k^{!} M \stackrel{d}{\longrightarrow} k_{!} k^{!} M \longrightarrow M \longrightarrow 0
$$

with

$$
\begin{aligned}
& d: \bigoplus_{\mu} k_{!} k^{!} M \rightarrow k_{!} k^{!} M ; x_{i} \otimes y \mapsto x_{i} z_{i} \otimes y-x_{i} \otimes z_{i} y \quad\left(x_{i} \in A\left[F_{\mu}\right], y \in M\right), \\
& k_{!} k^{!} M=k^{!} M\left[F_{\mu}\right] \rightarrow M ; x \otimes y \mapsto x y \quad\left(x \in A\left[F_{\mu}\right], y \in M\right),
\end{aligned}
$$

such that $d$ has augmentation $A$-module morphism

$$
\epsilon(d)=-\gamma_{M}: \bigoplus_{\mu} k^{!} M \rightarrow k^{!} M .
$$

Regarded as a right $A\left[F_{\mu}\right]$-module $A$ has a 1-dimensional f.g. free resolution

$$
0 \longrightarrow \bigoplus_{i=1}^{\mu} A\left[F_{\mu}\right] \stackrel{\oplus\left(z_{i}-1\right)}{\longrightarrow} A\left[F_{\mu}\right] \stackrel{\epsilon}{\longrightarrow} A \longrightarrow 0
$$

so that for any $A\left[F_{\mu}\right]$-module $M$

$$
\operatorname{Tor}_{n}^{A\left[F_{\mu}\right]}(A, M)= \begin{cases}A \otimes_{A\left[F_{\mu}\right]} M=\operatorname{coker}\left(\gamma_{M}\right) & \text { if } n=0, \\ \operatorname{ker}\left(\gamma_{M}\right) & \text { if } n=1, \\ 0 & \text { if } n \geqslant 2 .\end{cases}
$$

The equivalences (i) $\Longleftrightarrow$ (ii) $\Longleftrightarrow$ (iii) are now clear.

Definition 3.7 (i) Let $\mathcal{B l a} a_{\infty}(A)$ be the category of Blanchfield $A\left[F_{\mu}\right]$-modules, and let $\mathcal{B l a}(A) \subset \mathcal{B} l a_{\infty}(A)$ be the full subcategory of the h.d. 1 Blanchfield $A\left[F_{\mu}\right]-$ modules. (In view of Proposition $3.6 \mathcal{B} \operatorname{la}_{\infty}(A)$ is the same as the $F_{\mu}$-link module category $\mathcal{F} l k_{\infty}(A)$ of Sheiham [41]).

(ii) Let $\mathcal{F} l k(A) \subset \mathcal{B l a}(A)$ be the full subcategory of the h.d. 1 Blanchfield $A\left[F_{\mu}\right]-$ modules $M$ such that there exists a 1-dimensional induced $A\left[F_{\mu}\right]$-module resolution

$$
0 \longrightarrow P\left[F_{\mu}\right] \stackrel{d}{\longrightarrow} P\left[F_{\mu}\right] \longrightarrow M \longrightarrow 0
$$


with $P$ a f.g. projective $A$-module.

Example 3.8 (i) For a principal ideal domain $A$

$$
K_{0}\left(A\left[F_{\mu}\right]\right)=K_{0}(A)=\mathbb{Z}
$$

(see Bass [3]) so that

$$
\mathcal{B} l a(A)=\mathcal{F} l k(A) .
$$

(ii) A finitely presented Blanchfield $\mathbb{Z}\left[F_{\mu}\right]$-module is a 'type $L$ ' $\mathbb{Z}\left[F_{\mu}\right]$-module in the sense of Sato [37].

(iii) Given a $\mu$-component boundary link $\ell: \bigsqcup_{\mu} S^{n} \subset S^{n+2}$ let $c: W \rightarrow W_{0}$ be a $\mathbb{Z}$-homology equivalence from the exterior $W$ to the exterior $W_{0}$ of the trivial $\mu-$ component boundary link $\ell_{0}: \bigsqcup_{\mu} S^{n} \subset S^{n+2}$, with $F_{\mu}$-equivariant lift $\widetilde{c}: \widetilde{W} \rightarrow \widetilde{W}_{0}$ to the $F_{\mu}$-covers. The homology groups $\dot{H}_{*}(\widetilde{W})=H_{*+1}\left(\widetilde{c}: \widetilde{W} \rightarrow \widetilde{W}_{0}\right)$ are Blanchfield $\mathbb{Z}\left[F_{\mu}\right]$-modules of homological dimension $\leqslant 2$. Each $\dot{H}_{r}(\widetilde{W})$ has a $\mathbb{Z}$-contractible f.g. free $\mathbb{Z}\left[F_{\mu}\right]$-module resolution of the type

$$
0 \rightarrow \mathbb{Z}\left[F_{\mu}\right]^{a_{r}} \rightarrow \mathbb{Z}\left[F_{\mu}\right]^{b_{r}} \rightarrow \mathbb{Z}\left[F_{\mu}\right]^{c_{r}} \rightarrow \dot{H}_{r}(\widetilde{W}) \rightarrow 0 \quad(0 \leqslant r \leqslant n+1)
$$

with $a_{r}-b_{r}+c_{r}=0$, and $\dot{H}_{r}(\widetilde{W}) / \mathbb{Z}$-torsion is an h.d. $1 F_{\mu}$-link module (Levine $[26,3.5]$ for $\mu=1$, Sato [37, 3.1] and Duval [12,4.1] for $\mu \geqslant 2)$. See Example 3.13 below for the construction of an $(n+1)$-dimensional chain complex $C$ in $\operatorname{Sei}(\mathbb{Z})$ such that the covering $B(C)$ is an $(n+1)$-dimensional chain complex in $\mathcal{F} l k(\mathbb{Z})$ with $H_{*}(B(C))=\dot{H}_{*}(\widetilde{W})$.

The following Proposition 3.9 characterizes Blanchfield $A\left[F_{\mu}\right]$-modules in terms of $A\left[F_{\mu}\right]$-modules $K$ such that

$$
\operatorname{Tor}_{1}^{A\left[F_{\mu}\right]}(A, K)=0 .
$$

If $K$ is a flat $A\left[F_{\mu}\right]$-module then $\operatorname{Tor}_{1}^{A\left[F_{\mu}\right]}(B, K)=0$ for any right $A\left[F_{\mu}\right]$-module $B$, and in particular $B=A$. If $K=P\left[F_{\mu}\right]$ is induced from an $A$-module $P$ then

$$
\operatorname{Tor}_{1}^{A\left[F_{\mu}\right]}\left(A, P\left[F_{\mu}\right]\right)=\operatorname{Tor}_{1}^{A}(A, P)=0 .
$$

Proposition 3.9 (i) If $M$ is an $A\left[F_{\mu}\right]$-module with a resolution

$$
0 \longrightarrow K \stackrel{d}{\longrightarrow} L \longrightarrow M \longrightarrow 0
$$

such that

$$
\operatorname{Tor}_{1}^{A\left[F_{\mu}\right]}(A, K)=\operatorname{Tor}_{1}^{A\left[F_{\mu}\right]}(A, L)=0
$$


(e.g. the canonical Mayer-Vietoris presentation of Definition 2.10) then $M$ is Blanchfield if and only if the $A$-module morphism $1 \otimes d: A \otimes_{A\left[F_{\mu}\right]} K \rightarrow A \otimes_{A\left[F_{\mu}\right]} L$ is an isomorphism.

(ii) A morphism $d: K \rightarrow L$ of projective $A\left[F_{\mu}\right]$-modules is injective and $M=$ coker $(d)$ is a Blanchfield $A\left[F_{\mu}\right]$-module if and only if the $A$-module morphism $1 \otimes d: A \otimes_{A\left[F_{\mu}\right]} K \rightarrow A \otimes_{A\left[F_{\mu}\right]} L$ is an isomorphism.

Proof (i) It follows from Proposition 3.6 and the commutative diagram with exact rows and columns

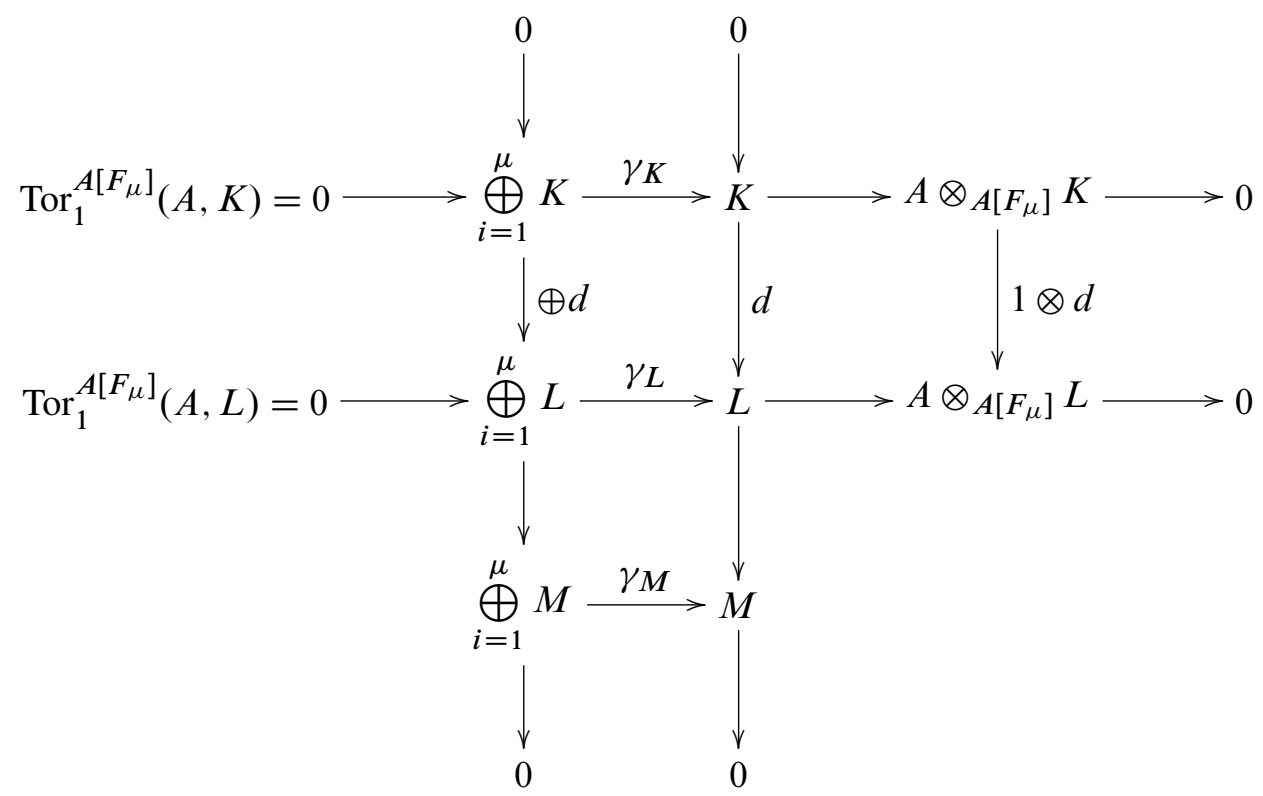

that $M$ is Blanchfield if and only if $1 \otimes d$ is an isomorphism.

(ii) If $d$ is injective and $M$ is Blanchfield then $1 \otimes d$ is an isomorphism by (ii), since projective $A\left[F_{\mu}\right]$-modules are flat. Conversely, if $1 \otimes d: A \otimes_{A\left[F_{\mu}\right]} K \rightarrow A \otimes_{A\left[F_{\mu}\right]} L$ is an isomorphism then $1 \otimes d: \widehat{A\left[F_{\mu}\right]} \otimes_{A\left[F_{\mu}\right]} K \rightarrow \widehat{A\left[F_{\mu}\right]} \otimes_{A\left[F_{\mu}\right]} L$ is an isomorphism by Proposition 3.1 (iii), and it follows from the injectivity of $K \rightarrow \widehat{A\left[F_{\mu}\right]} \otimes_{A\left[F_{\mu}\right]} K$, $L \rightarrow \widehat{A\left[F_{\mu}\right]} \otimes_{A\left[F_{\mu}\right]} L$ and the commutative diagram

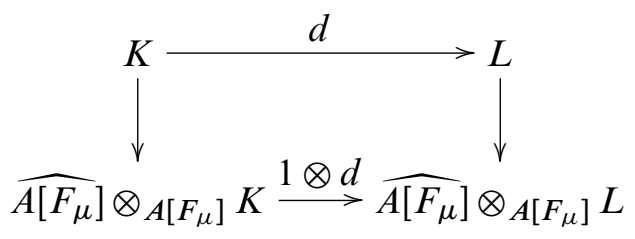


that $d: K \rightarrow L$ is injective.

The idempotent completion $\mathcal{P}(\mathcal{E})$ of an additive category $\mathcal{E}$ is the additive category with objects pairs $\left(M, p=p^{2}: M \rightarrow M\right)$ defined by projections $p$ of objects $M$ in $\mathcal{E}$, and morphisms $f:(M, p) \rightarrow(N, q)$ defined by morphisms $f: M \rightarrow N$ in $\mathcal{E}$ such that $q f p=f: M \rightarrow N$. As usual, $\mathcal{E}$ is idempotent complete if the functor $\mathcal{E} \rightarrow \mathcal{P}(\mathcal{E}) ; M \mapsto(M, 1)$ is an equivalence, or equivalently if for every idempotent $p=p^{2}: M \rightarrow M$ in $\mathcal{E}$ there exists a direct sum decomposition $M=P \oplus Q$ with

$$
p=\left(\begin{array}{ll}
1 & 0 \\
0 & 0
\end{array}\right): M=P \oplus Q \rightarrow M=P \oplus Q .
$$

For any exact category $\mathcal{E}$ there exists a full embedding $\mathcal{E} \subset \mathcal{A}$ in an abelian category $\mathcal{A}$ (Gabriel-Quillen), and the idempotent completion $\mathcal{P}(\mathcal{E})$ is equivalent to the full exact subcategory of $\mathcal{A}$ with objects $\operatorname{im}(p)$ for objects $(M, p)$ in $\mathcal{P}(\mathcal{E})$. For $\mathcal{E}=\mathcal{F} l k(A) \subset$ $\mathcal{A}=\mathcal{B} l a_{\infty}(A)$ we have that $\mathcal{P}(\mathcal{F} l k(A)) \subset \mathcal{B} l a_{\infty}(A)$. In fact, we have:

Proposition 3.10 (i) The exact categories $\operatorname{Prim}(A), \mathcal{S} e i(A), \mathcal{B} l a(A)$ are idempotent complete.

(ii) The idempotent completion of $\mathcal{F} l k(A)$ is equivalent to $\mathcal{B l a}(A)$

$$
\mathcal{P}(\mathcal{F} l k(A)) \approx \mathcal{B} l a(A)
$$

Proof (i) The exact categories $\operatorname{Prim}(A), \mathcal{S} e i(A), \mathcal{B l a}(A)$ are closed under direct summands.

(ii) For any f.g. projective $A\left[F_{\mu}\right]$-modules $K, L$ the augmentation map

$$
\epsilon: \operatorname{Hom}_{A\left[F_{\mu}\right]}(K, L) \rightarrow \operatorname{Hom}_{A}\left(A \otimes_{A\left[F_{\mu}\right]} K, A \otimes_{A\left[F_{\mu}\right]} L\right) ; d \mapsto 1 \otimes d
$$

is surjective, by the following argument: choose f.g. projective $A\left[F_{\mu}\right]$-modules $K^{\prime}, L^{\prime}$ such that

$$
K \oplus K^{\prime}=A\left[F_{\mu}\right]^{k}, L \oplus L^{\prime}=A\left[F_{\mu}\right]^{\ell}
$$

for some $k, \ell \geqslant 0$, and note that the augmentation map

$$
\begin{aligned}
\epsilon: \operatorname{Hom}_{A\left[F_{\mu}\right]}\left(K \oplus K^{\prime}, L \oplus L^{\prime}\right)=\operatorname{Hom}_{A\left[F_{\mu}\right]}\left(A\left[F_{\mu}\right]^{k}, A\left[F_{\mu}\right]^{\ell}\right) \\
\quad \longrightarrow \operatorname{Hom}_{A}\left(A \otimes_{A\left[F_{\mu}\right]}\left(K \oplus K^{\prime}\right), A \otimes_{A\left[F_{\mu}\right]}\left(L \oplus L^{\prime}\right)\right)=\operatorname{Hom}_{A}\left(A^{k}, A^{\ell}\right)
\end{aligned}
$$

is surjective. Given an h.d. 1 Blanchfield $A\left[F_{\mu}\right]$-module $M$ with a f.g. projective $A\left[F_{\mu}\right]$-module resolution

$$
0 \longrightarrow K \stackrel{d}{\longrightarrow} L \longrightarrow M \longrightarrow 0
$$


we know from Proposition 3.9 (i) that $1 \otimes d: A \otimes_{A\left[F_{\mu}\right]} K \rightarrow A \otimes_{A\left[F_{\mu}\right]} L$ is an $A-$ module isomorphism. By Proposition 3.1 (i) it is possible to lift $(1 \otimes d)^{-1}$ to an $A\left[F_{\mu}\right]$-module morphism $e: L \rightarrow K$, so that by Proposition 3.9 (i) $e$ is an injection with

$$
N=\operatorname{coker}(e)
$$

an h.d. 1 Blanchfield $A\left[F_{\mu}\right]$-module. Let $J$ be a f.g. projective $A\left[F_{\mu}\right]$-module such that $J \oplus K \oplus L$ is f.g. free, say $A\left[F_{\mu}\right]^{m}$. The $A\left[F_{\mu}\right]$-module morphism

$$
f=1 \oplus\left(\begin{array}{ll}
0 & e \\
d & 0
\end{array}\right): J \oplus K \oplus L=A\left[F_{\mu}\right]^{m} \rightarrow J \oplus K \oplus L=A\left[F_{\mu}\right]^{m}
$$

is such that $1 \otimes f: A^{m} \rightarrow A^{m}$ is an isomorphism, so that $\operatorname{coker}(f)=M \oplus N$ is an h.d. $1 F_{\mu}$-link module. The functor

$$
\mathcal{F} l k(A) \rightarrow \mathcal{B l a}(A) ; \quad M \mapsto M
$$

is a full embedding such that every object in $\mathcal{B l a}(A)$ is a direct summand of an object in $\mathcal{F} l k(A)$, so that $\mathcal{B} l a(A)$ is (equivalent to) the idempotent completion $\mathcal{P}(\mathcal{F} l k(A))$.

\subsection{Seifert modules}

Let $Q_{\mu}$ be the complete quiver which has $\mu$ vertices and $\mu^{2}$ arrows, one arrow between each ordered pair of vertices. The path ring is given by

$$
Q_{\mu}=\mathbb{Z}[e] * \mathbb{Z}\left[\pi_{1}, \pi_{2}, \ldots, \pi_{\mu} \mid \pi_{i} \pi_{j}=\delta_{i j} \pi_{i}, \sum_{i=1}^{\mu} \pi_{i}=1\right]
$$

where $\pi_{i} e \pi_{j}$ corresponds to the unique path of length 1 from the $i$ th vertex to the $j$ th vertex. An $A$-module $P$ together with a ring morphism $\rho: Q_{\mu} \rightarrow \operatorname{End}_{A}(P)$ is essentially the same as a triple $\left(P, e,\left\{\pi_{i}\right\}\right)$ with $e: P \rightarrow P$ an endomorphism, and $\left\{\pi_{i}: P \rightarrow P\right\}$ a complete system of $\mu$ idempotents. (Such representations of $Q_{\mu}$ were first considered by Farber [14] for particular $A$.)

Definition 3.11 (i) A Seifert $A$-module $\left(P, e,\left\{\pi_{i}\right\}\right)$ is an $A$-module $P$ together with an endomorphism $e: P \rightarrow P$, and a system $\left\{\pi_{i}: P \rightarrow P\right\}$ of idempotents expressing $P$ as a $\mu$-fold direct sum, with

$$
\pi_{i}: P=P_{1} \oplus P_{2} \oplus \cdots \oplus P_{\mu} \rightarrow P ;\left(x_{1}, x_{2}, \ldots, x_{\mu}\right) \mapsto\left(0, \ldots, 0, x_{i}, 0, \ldots, 0\right) .
$$

(ii) A morphism of Seifert $A$-modules

$$
g:\left(P, e,\left\{\pi_{i}\right\}\right) \rightarrow\left(P^{\prime}, e^{\prime},\left\{\pi_{i}^{\prime}\right\}\right)
$$

is an $A$-module morphism such that

$$
g e=e^{\prime} g, g \pi_{i}=\pi_{i}^{\prime} g: P \rightarrow P^{\prime} .
$$


The conditions $g \pi_{i}=\pi_{i}^{\prime} g$ are equivalent to $g$ preserving the direct sum decompositions, so that

$$
g=\left(\begin{array}{cccc}
g_{1} & 0 & \ldots & 0 \\
0 & g_{2} & \ldots & 0 \\
0 & 0 & \ldots & 0 \\
\vdots & \vdots & \ddots & 0 \\
0 & 0 & \ldots & g_{\mu}
\end{array}\right): P=P_{1} \oplus P_{2} \oplus \cdots \oplus P_{\mu} \rightarrow P^{\prime}=P_{1}^{\prime} \oplus P_{2}^{\prime} \oplus \cdots \oplus P_{\mu}^{\prime}
$$

with $g_{i}: P_{i} \rightarrow P_{i}^{\prime}$.

(iii) The Seifert $A$-module category $\mathcal{S e i}_{\infty}(A)$ has objects Seifert $A$-modules and morphisms as in (ii). Let $\operatorname{Sei}(A) \subseteq \mathcal{S e i}_{\infty}(A)$ be the full subcategory of the Seifert $A$-modules $\left(P, e,\left\{\pi_{i}\right\}\right)$ with $P$ f.g. projective.

\subsection{The covering functor $B$}

Seifert modules determine $F_{\mu}$-link modules by:

Definition 3.12 (i) The covering of a Seifert $A$-module $\left(P, e,\left\{\pi_{i}\right\}\right)$ is the $F_{\mu}$-link module

$$
B\left(P, e,\left\{\pi_{i}\right\}\right)=\operatorname{coker}\left(1-e+e z: P\left[F_{\mu}\right] \rightarrow P\left[F_{\mu}\right]\right)
$$

with Mayer-Vietoris presentation

$$
0 \longrightarrow \bigoplus_{i=1}^{\mu} P_{i}\left[F_{\mu}\right] \stackrel{d}{\longrightarrow} P\left[F_{\mu}\right] \longrightarrow B\left(P, e,\left\{\pi_{i}\right\}\right) \longrightarrow 0,
$$

where $d=1-e+e z$.

(ii) The covering of a Seifert $A$-module morphism $g:\left(P, e,\left\{\pi_{i}\right\}\right) \rightarrow\left(P^{\prime}, e^{\prime},\left\{\pi_{i}^{\prime}\right\}\right)$ is the $F_{\mu}$-link module morphism

$$
B(g): B\left(P, e,\left\{\pi_{i}\right\}\right) \rightarrow B\left(P^{\prime}, e^{\prime},\left\{\pi_{i}^{\prime}\right\}\right) ; x \mapsto g(x)
$$

resolved by

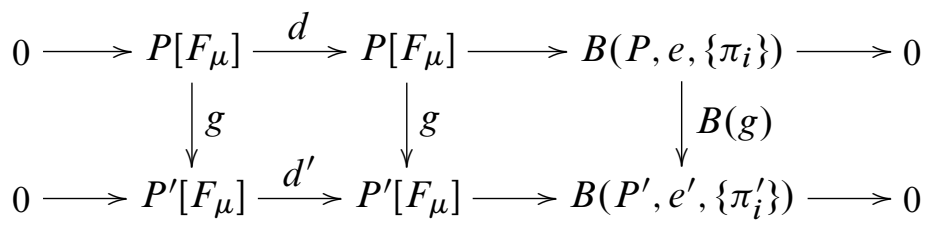

with $d=1-e+e z, d^{\prime}=1-e^{\prime}+e^{\prime} z$. 
Example 3.13 Let $\ell$ : $\bigsqcup_{\mu} S^{n} \subset S^{n+2}$ be a $\mu$-component boundary link with exterior $W$, so that there exists a $\mathbb{Z}$-homology equivalence $c: W \rightarrow W_{0}$ to the exterior $W_{0}$ of the trivial $\mu$-component boundary link $\ell_{0}: \bigsqcup_{\mu} S^{n} \subset S^{n+2}$. The $(n+2)$-dimensional f.g. free $\mathbb{Z}\left[F_{\mu}\right]$-module chain complex

$$
\dot{C}(\widetilde{W})=\mathcal{C}\left(\widetilde{c}: C(\widetilde{W}) \rightarrow C\left(\widetilde{W}_{0}\right)\right)_{*+1}
$$

is $\mathbb{Z}$-contractible. For any $\mu$-component Seifert surface $V=V_{1} \sqcup V_{2} \sqcup \ldots \sqcup V_{\mu} \subset S^{n+2}$ for $\ell$ there exists a degree 1 map $V \rightarrow V_{0}$ to the $\mu$-component Seifert surface $V_{0}=\bigsqcup_{\mu} D^{n+1} \subset S^{n+2}$ for $\ell_{0}$. Let

$$
\dot{C}\left(V_{i}\right)=\mathcal{C}\left(C\left(V_{i}\right) \rightarrow C\left(D^{n+1}\right)\right)_{*+1}, \quad \dot{C}(V)=\sum_{i=1}^{\mu} \dot{C}\left(V_{i}\right) .
$$

The map $V \rightarrow S^{n+2} \backslash V$ pushing $V$ off itself in the positive normal direction combines with chain level Alexander duality to induce a $\mathbb{Z}$-module chain map

$$
e: \dot{C}(V) \rightarrow C\left(S^{n+2} \backslash V, \bigsqcup_{\mu}\{\mathrm{pt} .\}\right) \simeq \dot{C}(V)^{n+1-*},
$$

so that there is defined an $(n+1)$-dimensional chain complex $\left(\dot{C}(V), e,\left\{\pi_{i}\right\}\right)$ in $\operatorname{Sei}(\mathbb{Z})$. The covering $B\left(\dot{C}(V), e,\left\{\pi_{i}\right\}\right)$ is an $(n+1)$-dimensional chain complex in $\mathcal{F} l k(\mathbb{Z})$, with the projection

$$
\begin{aligned}
\mathcal{C}\left(1-e+e z: \dot{C}(V)\left[F_{\mu}\right] \rightarrow \dot{C}(V)\left[F_{\mu}\right]\right) & =\dot{C}(\widetilde{W}) \\
& \longrightarrow B\left(\dot{C}(V), e,\left\{\pi_{i}\right\}\right)=\operatorname{coker}\left(1-e+e z: \dot{C}(V)\left[F_{\mu}\right] \rightarrow \dot{C}(V)\left[F_{\mu}\right]\right)
\end{aligned}
$$

a homology equivalence.

The covering construction defines a functor of exact categories

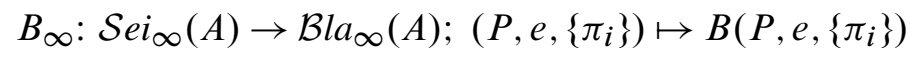

which restricts to a functor $B: \mathcal{S e i}(A) \rightarrow \mathcal{F} l k(A)$.

Definition 3.14 A morphism $f$ in $\mathcal{S e i}_{\infty}(A)$ is a $B$-isomorphism if $B(f)$ is an isomorphism in $\mathcal{B} l a_{\infty}(A)$. Let $\Xi_{\infty}$ denote the set of $B$-isomorphisms in $\mathcal{S e i}_{\infty}(A)$, and let $\Xi$ denote the set of $B$-isomorphisms in $\operatorname{Sei}(A)$.

\subsection{Blanchfield/Seifert algebraic transversality}

We shall now use the algebraic transversality of Section 2 to establish that every h.d. 1 $F_{\mu}$-link module $M$ is isomorphic to the covering $B\left(P, e,\left\{\pi_{i}\right\}\right)$ of a f.g. projective Seifert $A$-module $\left(P, e,\left\{\pi_{i}\right\}\right)$, uniquely up to morphisms in $\Xi$. 
We refer to Sheiham [41] for the proof that $B_{\infty}: \mathcal{S} e i_{\infty}(A) \rightarrow \mathcal{F} l k_{\infty}(A)$ induces an equivalence of exact categories $\bar{B}_{\infty}: \Xi_{\infty}^{-1} \mathcal{S} e i_{\infty}(A) \approx \mathcal{F} l k_{\infty}(A)$. Algebraic transversality will be used to prove that the universal localization $\operatorname{Sei}(A) \rightarrow \Xi^{-1} \mathcal{S e i}(A)$ has a calculus of fractions, and that the covering functor $B: \operatorname{Sei}(A) \rightarrow \mathcal{F} l k(A)$ induces an equivalence of exact categories $\bar{B}: \Xi^{-1} \mathcal{S e i}(A) \approx \mathcal{F} l k(A)$.

Given an $F_{\mu}$-link module $M$ let $U(M)=\left(M, e_{M},\left\{\pi_{i}\right\}\right)$ be the Seifert $A$-module defined in [41] - the definition is recalled in the Introduction of this paper, along with the fact proved in [41] that $B_{\infty}$ is a left adjoint of

$$
U_{\infty}: \mathcal{B l a}_{\infty}(A) \rightarrow \mathcal{S} e i_{\infty}(A) ; M \mapsto U(M) .
$$

The natural isomorphism of the adjointness

$$
\begin{aligned}
\operatorname{Hom}_{\mathcal{B l a} \infty}(A)\left(B\left(Q, f,\left\{\rho_{i}\right\}\right), M\right) & \cong \operatorname{Hom}_{\mathcal{S} e i_{\infty}(A)}\left(\left(Q, f,\left\{\rho_{i}\right\}\right), U(M)\right) ; \\
g & \longmapsto \operatorname{adj}(g)=U(g) h
\end{aligned}
$$

is defined for any Seifert $A$-module $\left(Q, f,\left\{\rho_{i}\right\}\right)$, with

$$
h: Q \subset Q\left[F_{\mu}\right] \rightarrow U B\left(Q, f,\left\{\rho_{i}\right\}\right)
$$

the restriction of the canonical surjection $Q\left[F_{\mu}\right] \rightarrow B\left(Q, f,\left\{\rho_{i}\right\}\right)$. If $M$ is h.d. 1 and $\left(Q, f,\left\{\rho_{i}\right\}\right)$ is f.g. projective the natural isomorphism can be written as

$$
\operatorname{Hom}_{\mathcal{F} l k(A)}\left(B\left(Q, f,\left\{\rho_{i}\right\}\right), M\right) \cong \operatorname{Hom}_{\mathcal{S} e i_{\infty}(A)}\left(\left(Q, f,\left\{\rho_{i}\right\}\right), U(M)\right)
$$

but note that in general $U(M)$ is not a f.g. projective Seifert $A$-module.

The following result establishes that for an h.d. $1 F_{\mu}$-link module $M$ the Seifert $A$-module $U(M)$ is the direct limit of a directed system of f.g. projective Seifert $A-$ modules $\left(P, e,\left\{\pi_{i}\right\}\right)$ and morphisms in $\Xi$, with isomorphisms $B\left(P, e,\left\{\pi_{i}\right\}\right) \cong M$.

Theorem 3.15 (Blanchfield/Seifert algebraic transversality) Let $M$ be an h.d. $1 F_{\mu}-$ link module, with a 1 -dimensional induced f.g. projective $A\left[F_{\mu}\right]$-module resolution

$$
0 \longrightarrow P\left[F_{\mu}\right] \stackrel{d}{\longrightarrow} P\left[F_{\mu}\right] \longrightarrow M \longrightarrow 0
$$

such that $\epsilon(d): P \rightarrow P$ is an $A$-module isomorphism.

(i) Let $I_{\infty}$ be the set of ordered pairs $T=\left(T_{0}, T_{1}\right)$ of subtrees $T_{0}, T_{1} \subseteq G_{\mu}$ such that $d_{*}\left(T_{1}\right) \subseteq T_{0}$. The set $I_{\infty}$ is partially ordered by inclusion, with maximal element

$$
T_{\max }=\bigcup_{T \in I_{\infty}} T=\left(G_{\mu}, G_{\mu}\right) \in I_{\infty}
$$


There is defined a directed system of Seifert $A$-modules $\left(P\langle T\rangle, e\langle T\rangle,\left\{\pi_{i}\langle T\rangle\right\}\right)$ and morphisms in $\Xi_{\infty}$

$$
\phi\left\langle T, T^{\prime}\right\rangle:\left(P\langle T\rangle, e\langle T\rangle,\left\{\pi_{i}\langle T\rangle\right\}\right) \longrightarrow\left(P\left\langle T^{\prime}\right\rangle, e\left\langle T^{\prime}\right\rangle,\left\{\pi_{i}\left\langle T^{\prime}\right\rangle\right\}\right) \quad\left(T \subseteq T^{\prime} \in I_{\infty}\right)
$$

with direct limit

$$
\underset{T \in I_{\infty}}{\lim _{\infty}}\left(P\langle T\rangle, e\langle T\rangle,\left\{\pi_{i}\langle T\rangle\right\}\right)=\left(P\left\langle T_{\max }\right\rangle, e\left\langle T_{\max }\right\rangle,\left\{\pi_{i}\left\langle T_{\max }\right\rangle\right\}\right)=U(M) .
$$

For any $T=\left(T_{0}, T_{1}\right) \in I_{\infty}$ the morphism $\phi\left\langle T, T_{\max }\right\rangle:\left(P\langle T\rangle, e\langle T\rangle,\left\{\pi_{i}\langle T\rangle\right\}\right) \rightarrow$ $U(M)$ is the adjoint $\phi\left\langle T, T_{\max }\right\rangle=\operatorname{adj}(\phi\langle T\rangle)$ of an isomorphism in $\mathcal{F} l k(A)$

$$
\phi\langle T\rangle: B\left(P\langle T\rangle, e\langle T\rangle,\left\{\pi_{i}\langle T\rangle\right\}\right) \stackrel{\cong}{\longrightarrow} M
$$

such that for any $T \subseteq T^{\prime} \in I_{\infty}$ there is defined a commutative triangle of isomorphisms in $\mathcal{F} l k_{\infty}(A)$

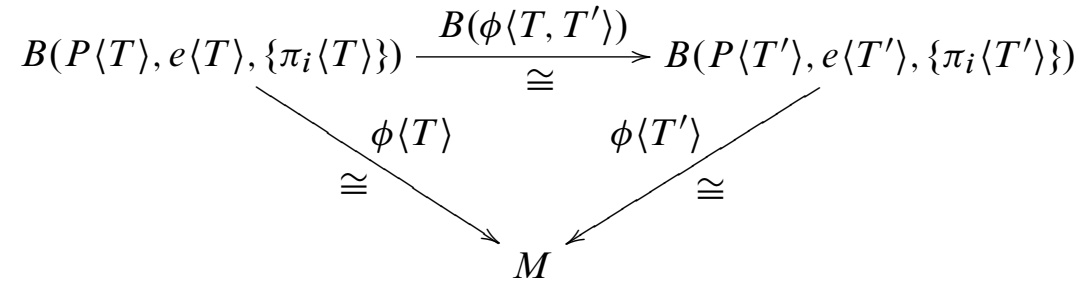

In particular, $\phi\left\langle T, T_{\max }\right\rangle \in \Xi_{\infty}$.

(ii) Let $I \subset I_{\infty}$ be the subset of the ordered pairs $T=\left(T_{0}, T_{1}\right)$ of finite subtrees $T_{0}, T_{1} \subset G_{\mu}$ such that $d_{*} T_{1} \subseteq T_{0}$. For $T \in I\left(P\langle T\rangle, e\langle T\rangle,\left\{\pi_{i}\langle T\rangle\right\}\right)$ is a f.g. projective Seifert $A$-module, and

$$
\underset{\overrightarrow{T \in I}}{\lim _{\overrightarrow{1}}}\left(P\langle T\rangle, e\langle T\rangle,\left\{\pi_{i}\langle T\rangle\right\}\right)=U(M)
$$

with $\phi\left\langle T, T^{\prime}\right\rangle \in \Xi\left(T \subseteq T^{\prime} \in I\right)$.

(iii) For any f.g. projective Seifert $A$-module $\left(Q, f,\left\{\rho_{i}\right\}\right)$ every morphism

$$
g: B\left(Q, f,\left\{\rho_{i}\right\}\right) \rightarrow M
$$

in $\mathcal{F l k}(A)$ factors as

$$
g: B\left(Q, f,\left\{\rho_{i}\right\}\right) \stackrel{B(g\langle T\rangle)}{\longrightarrow} B\left(P\langle T\rangle, e\langle T\rangle,\left\{\pi_{i}\langle T\rangle\right\}\right) \stackrel{\phi\langle T\rangle}{\cong} M
$$

for some $T \in I$, with $g\langle T\rangle:\left(Q, f,\left\{\rho_{i}\right\}\right) \rightarrow\left(P\langle T\rangle, e\langle T\rangle,\left\{\pi_{i}\langle T\rangle\right\}\right)$ a morphism in $\operatorname{Sei}(A)$. 
Proof (i) The induced f.g. projective $A\left[F_{\mu}\right]$-module chain complex

$$
E: E_{1}=P\left[F_{\mu}\right] \stackrel{d}{\longrightarrow} E_{0}=P\left[F_{\mu}\right]
$$

is such that $H_{0}(E)=M, H_{1}(E)=0$. By Theorem 2.12 for any subtree $T_{1} \subseteq G_{\mu}$ there exists a subtree $d_{*}\left(T_{1}\right) \subseteq G_{\mu}$ such that for any subtree $T_{0} \subseteq G_{\mu}$ with $d_{*}\left(T_{1}\right) \subseteq T_{0}$ $E$ admits a Mayer-Vietoris presentation

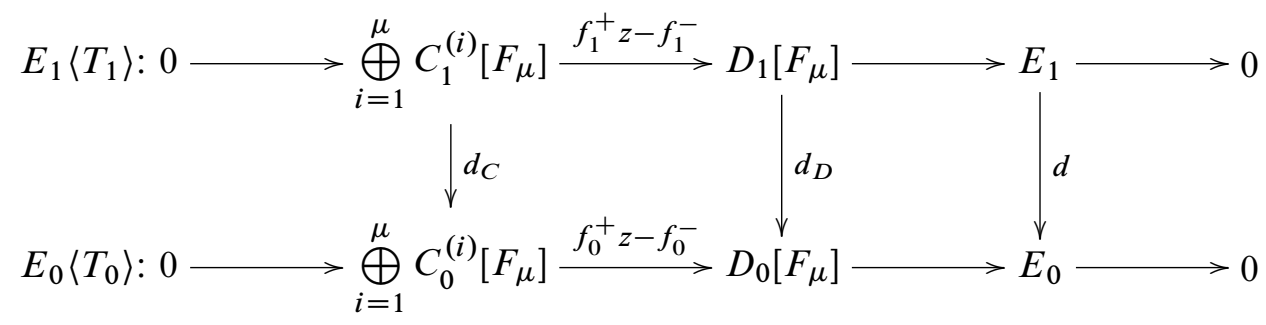

with $C_{j}^{(i)}=P\left[T_{j}^{(i, 1)}\right], \quad D_{j}=P\left[T_{j}^{(0)}\right] \subseteq E_{j}=P\left[F_{\mu}\right] \quad(j=0,1)$,

and $\quad d_{C}=\bigoplus_{i=1}^{\mu} d\left|: \bigoplus_{i=1}^{\mu} C_{1}^{(i)} \rightarrow \bigoplus_{i=1}^{\mu} C_{0}^{(i)}, \quad d_{D}=d\right|: D_{1} \rightarrow D_{0}$.

The $A$-modules defined by

$$
\begin{aligned}
P_{i}\langle T\rangle & =\operatorname{coker}\left(d \mid: C_{1}^{(i)} \rightarrow C_{0}^{(i)}\right), \\
P\langle T\rangle & =\operatorname{coker}\left(d_{C}\right)=\bigoplus_{i=1}^{\mu} P_{i}\langle T\rangle, \\
Q\langle T\rangle & =\operatorname{coker}\left(d_{D}\right)
\end{aligned}
$$

fit into a commutative diagram of $A\left[F_{\mu}\right]$-modules with exact rows and columns

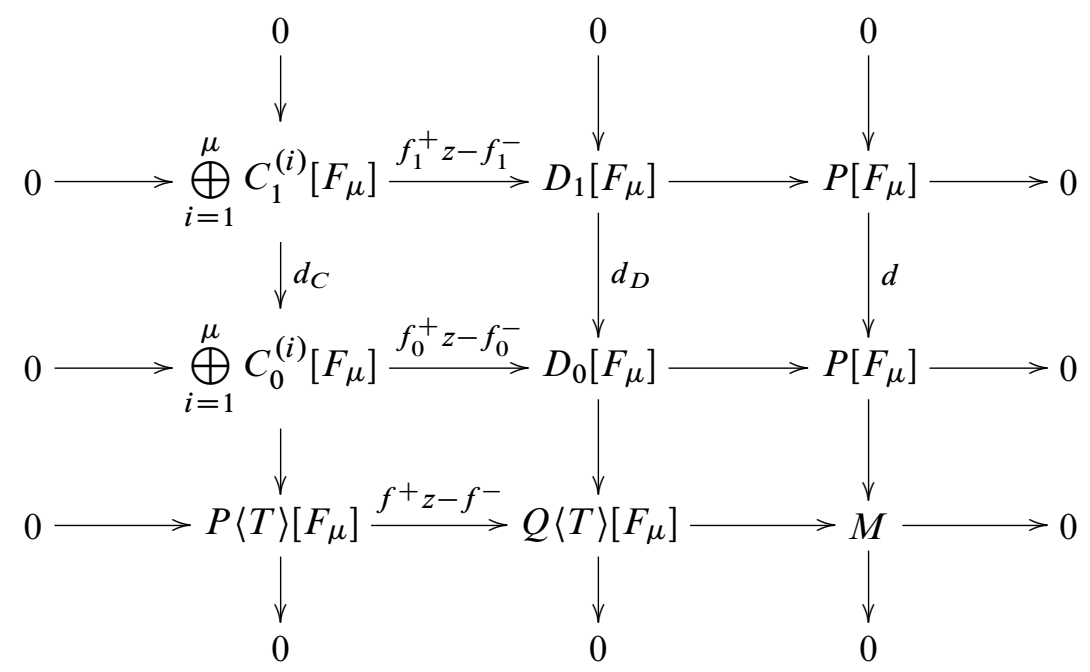


with $f^{+}, f^{-}: P\langle T\rangle \rightarrow Q\langle T\rangle$ the $A$-module morphisms induced by

$$
f_{0}^{+}, f_{0}^{-}: \bigoplus_{i=1}^{\mu} C_{0}^{(i)} \rightarrow D_{0} .
$$

It follows from $\operatorname{Tor}_{1}^{A\left[F_{\mu}\right]}(A, M)=0$ that $f^{+}-f^{-}: P\langle T\rangle \rightarrow Q\langle T\rangle$ is an $A$-module isomorphism. The Seifert $A$-module $\left(P\langle T\rangle, e\langle T\rangle,\left\{\pi_{i}\langle T\rangle\right\}\right)$ defined by

$$
e\langle T\rangle=\left(f^{+}-f^{-}\right)^{-1} f^{+}: P\langle T\rangle \rightarrow P\langle T\rangle, \quad \pi_{i}\langle T\rangle: P\langle T\rangle \rightarrow P_{i}\langle T\rangle \rightarrow P\langle T\rangle
$$

is such that $P\langle T\rangle\left[F_{\mu}\right] \cong Q\langle T\rangle\left[F_{\mu}\right] \rightarrow M$ induces the isomorphism of Blanchfield $A\left[F_{\mu}\right]$-modules $\phi\langle T\rangle: B\left(P\langle T\rangle, e\langle T\rangle,\left\{\pi_{i}\langle T\rangle\right\}\right) \cong M$ adjoint to the natural map $\left(P\langle T\rangle, e\langle T\rangle,\left\{\pi_{i}\langle T\rangle\right\}\right) \rightarrow U(M)$. (In particular, $\left(P\left\langle T_{\max }\right\rangle, e\left\langle T_{\max }\right\rangle,\left\{\pi_{i}\left\langle T_{\max }\right\rangle\right\}\right)=$ $U(M)$ and $\phi\left\langle T_{\max }\right\rangle: B U(M) \cong M$ is the natural isomorphism $\psi_{M}$ defined in [41, 5.10].) For $T \subseteq T^{\prime} \in I$ the $B$-isomorphism $\phi\left\langle T, T^{\prime}\right\rangle$ is induced by the inclusion $T \subseteq T^{\prime}$

(ii) The augmentation of the $A\left[F_{\mu}\right]$-module morphism $d: P\left[F_{\mu}\right] \rightarrow P\left[F_{\mu}\right]$ is an $A$-module isomorphism $\epsilon(d): P \rightarrow P$, so that the induced $\widehat{A\left[F_{\mu}\right]}$-module morphism $\widehat{d}: \widehat{P\left[F_{\mu}\right]} \rightarrow \widehat{P\left[F_{\mu}\right]}$ is an isomorphism, by Proposition 3.1. For any $T=\left(T_{0}, T_{1}\right) \in I$ the inclusion $P\left[T_{1}^{(0)}\right] \rightarrow \widehat{P\left[F_{\mu}\right]}$ is a split $A$-module injection by Proposition 3.3. Let $s: \widehat{P\left[F_{\mu}\right]} \rightarrow P\left[T_{1}^{(0)}\right]$ be a splitting $A$-module surjection. The anticlockwise composition of the morphisms (inverting $\widehat{d}$ ) in the diagram

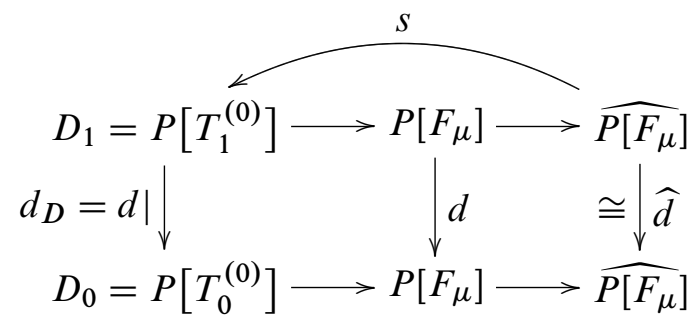

defines an $A$-module surjection $P\left[T_{0}^{(0)}\right] \rightarrow P\left[T_{1}^{(0)}\right]$ splitting $d \mid: P\left[T_{1}^{(0)}\right] \rightarrow P\left[T_{0}^{(0)}\right]$. Thus $d \mid$ is a split injection of f.g. projective $A$-modules and $P\langle T\rangle=\operatorname{coker}(d \mid)$ is a f.g. projective $A$-module.

(iii) The morphism $g: B\left(Q, f,\left\{\rho_{i}\right\}\right) \rightarrow M$ in $\mathcal{F} l k(A)$ has a canonical resolution

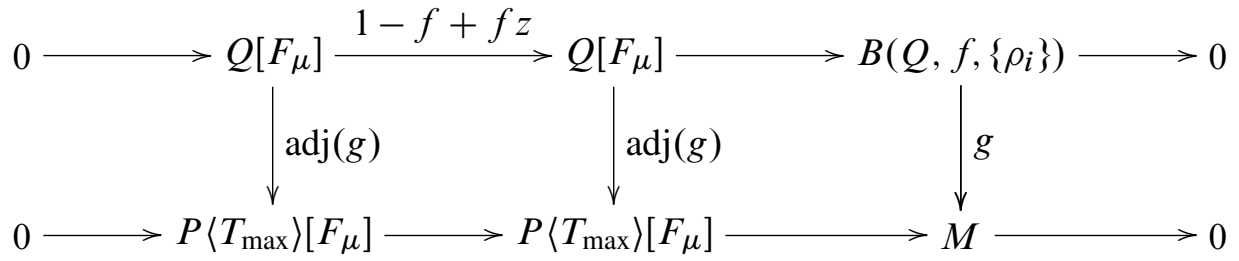


with

$$
\operatorname{adj}(g):\left(Q, f,\left\{\rho_{i}\right\}\right) \rightarrow\left(P\left\langle T_{\max }\right\rangle, e\left\langle T_{\max }\right\rangle,\left\{\pi_{i}\left\langle T_{\max }\right\rangle\right\}\right)=U(M)
$$

the adjoint morphism in $\mathcal{S e i}_{\infty}(A)$. Since $Q$ is f.g. projective there exists $T \in I$ such that

$$
\operatorname{im}\left(g: B\left(Q, f,\left\{\rho_{i}\right\}\right) \rightarrow M\right) \subseteq \operatorname{im}\left(B\left(P\langle T\rangle, e\langle T\rangle,\left\{\pi_{i}\langle T\rangle\right\}\right) \rightarrow M\right)
$$

with a lift of $g$ to an $A$-module morphism $g\langle T\rangle: Q \rightarrow P\langle T\rangle$ which preserves the direct sum structures. The diagram of $A$-modules and morphisms

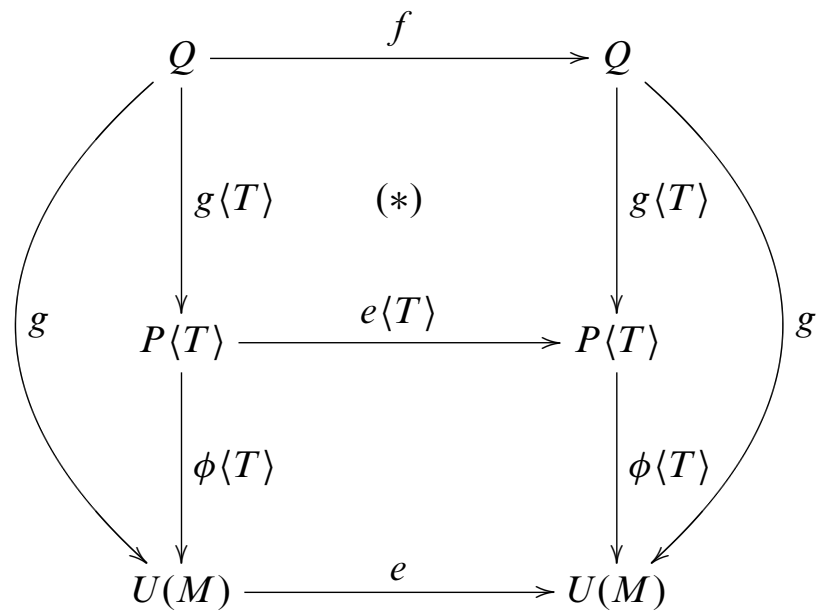

commutes except possibly in $(*)$, and $(*)$ commutes if and only if

$$
g\langle T\rangle:\left(Q, f,\left\{\rho_{i}\right\}\right) \rightarrow\left(P\langle T\rangle, e\langle T\rangle,\left\{\pi_{i}\langle T\rangle\right\}\right)
$$

is a morphism of Seifert $A$-modules. Since $Q$ is f.g. projective and the composite

$$
Q \stackrel{g\langle T\rangle f-e\langle T\rangle g\langle T\rangle}{\longrightarrow} P\langle T\rangle \stackrel{\phi\langle T\rangle}{\longrightarrow} U(M)=\underset{T^{\prime} \in I}{\lim } P\left\langle T^{\prime}\right\rangle
$$

is 0 there exists $T^{\prime} \in I$ such that $T \subseteq T^{\prime}$ and the composite

$$
g\left\langle T^{\prime}\right\rangle f-e\left\langle T^{\prime}\right\rangle g\left\langle T^{\prime}\right\rangle: Q \stackrel{g\langle T\rangle f-e\langle T\rangle g\langle T\rangle}{\longrightarrow} P\langle T\rangle \longrightarrow P\left\langle T^{\prime}\right\rangle
$$

is 0 , so that

$$
g\left\langle T^{\prime}\right\rangle:\left(Q, f,\left\{\rho_{i}\right\}\right) \rightarrow\left(P\left\langle T^{\prime}\right\rangle, e\left\langle T^{\prime}\right\rangle,\left\{\pi_{i}\left\langle T^{\prime}\right\rangle\right\}\right)
$$

is a morphism of Seifert $A$-modules as required (except that $T^{\prime}$ has to be called $T$ ).

Definition 3.16 Let $M=B\left(P, e,\left\{\pi_{i}\right\}\right)$ for a f.g. projective Seifert $A$-module $\left(P, e,\left\{\pi_{i}\right\}\right)$. 
(i) For any $T \in I_{\infty}$ let

$$
s\langle T\rangle:\left(P, e,\left\{\pi_{i}\right\}\right) \rightarrow\left(P\langle T\rangle, e\langle T\rangle,\left\{\pi_{i}\langle T\rangle\right\}\right)
$$

be the $B$-isomorphism determined by the inclusion $P=P[\{1\}] \subseteq P\left[T_{0}^{(0)}\right]$.

(ii) For $T=T_{\max } \in I_{\infty}$ write

$$
s_{M}=s\left\langle T_{\max }\right\rangle:\left(P, e,\left\{\pi_{i}\right\}\right) \rightarrow\left(P\left\langle T_{\max }\right\rangle, e\left\langle T_{\max }\right\rangle,\left\{\pi_{i}\left\langle T_{\max }\right\rangle\right\}\right)=U(M) .
$$

This is the $B$-isomorphism adjoint of $1: M \rightarrow M$, such that

$$
s_{M}:\left(P, e,\left\{\pi_{i}\right\}\right) \stackrel{s\langle T\rangle}{\longrightarrow}\left(P\langle T\rangle, e,\left\{\pi_{i}\right\}\right) \stackrel{\phi\langle T\rangle}{\longrightarrow} U(M)
$$

for any $T \in I_{\infty}$.

Putting everything together:

Theorem 3.17 (i) Every h.d. $1 F_{\mu}$-link module $M$ is isomorphic to the covering $B\left(P, e,\left\{\pi_{i}\right\}\right)$ of a f.g. projective Seifert $A$-module $\left(P, e,\left\{\pi_{i}\right\}\right)$.

(ii) For any f.g. projective Seifert $A$-modules $\left(P, e,\left\{\pi_{i}\right\}\right),\left(Q, f,\left\{\rho_{i}\right\}\right)$ every morphism $g: B\left(Q, f,\left\{\rho_{i}\right\}\right) \rightarrow B\left(P, e,\left\{\pi_{i}\right\}\right)$ in $\mathcal{F} l k(A)$ is of the form $g=B(s)^{-1} B(t)$ for some morphisms

$$
s:\left(P, e,\left\{\pi_{i}\right\}\right) \rightarrow\left(P^{\prime}, e^{\prime},\left\{\pi_{i}^{\prime}\right\}\right), t:\left(Q, f,\left\{\rho_{i}\right\}\right) \rightarrow\left(P^{\prime}, e^{\prime},\left\{\pi_{i}^{\prime}\right\}\right)
$$

in $\operatorname{Sei}(A)$ with $s \in \Xi$.

(iii) If $u:\left(Q, f,\left\{\rho_{i}\right\}\right) \rightarrow\left(P, e,\left\{\pi_{i}\right\}\right)$ is a morphism of f.g. projective Seifert $A-$ modules such that $B(u)=0$ there exists an element $v:\left(P, e,\left\{\pi_{i}\right\}\right) \rightarrow\left(P^{\prime}, e^{\prime},\left\{\pi_{i}^{\prime}\right\}\right)$ in $\Xi$ such that $v u=0$.

(iv) The localization $\Xi^{-1} \mathcal{S e i}(A)$ has a left calculus of fractions, and the covering construction defines an equivalence of exact categories

$$
\bar{B}: \Xi^{-1} \mathcal{S} e i(A) \stackrel{\approx}{\longrightarrow} \mathcal{F} l k(A) ;\left(P, e,\left\{\pi_{i}\right\}\right) \mapsto B\left(P, e,\left\{\pi_{i}\right\}\right) .
$$

Proof (i) By Theorem 3.15 (i)-(ii) $M$ is isomorphic to $B\left(P\langle T\rangle, e\langle T\rangle,\left\{\pi_{i}\langle T\rangle\right\}\right)$ for any $T \in I$, e.g. for the minimal element $T_{\min }=\left(d_{*}\{1\},\{1\}\right) \in I$.

(ii) By Theorem 3.15 (iii) the adjoint of $g$ factors in $\mathcal{S} e i_{\infty}(A)$ as

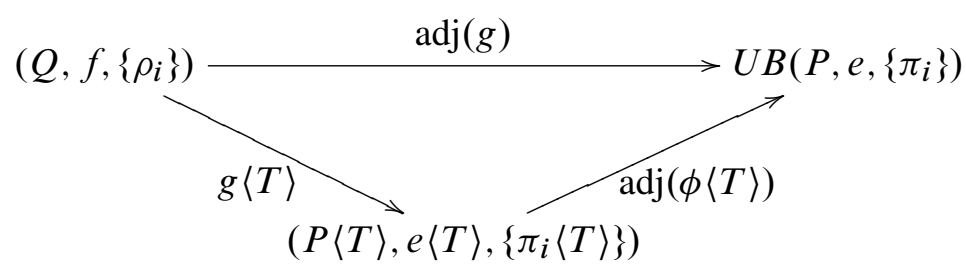


for some $T \in I$. The morphisms in $\operatorname{Sei}(A)$ defined by

$$
\begin{aligned}
& s=s\langle T\rangle:\left(P, e,\left\{\pi_{i}\right\}\right) \rightarrow\left(P^{\prime}, e^{\prime},\left\{\pi_{i}^{\prime}\right\}\right)=\left(P\langle T\rangle, e\langle T\rangle,\left\{\pi_{i}\langle T\rangle\right\}\right) \\
& t=g\langle T\rangle:\left(Q, f,\left\{\rho_{i}\right\}\right) \rightarrow\left(P^{\prime}, e^{\prime},\left\{\pi_{i}^{\prime}\right\}\right)=\left(P\langle T\rangle, e\langle T\rangle,\left\{\pi_{i}\langle T\rangle\right\}\right)
\end{aligned}
$$

are such that $s$ is a $B$-isomorphism (ie $s \in \Xi$ ) and $g=B(s)^{-1} B(t)$.

(iii) Let $M=B\left(P, e,\left\{\pi_{i}\right\}\right)$. We have a commutative diagram in $\mathcal{S} e i_{\infty}(A)$

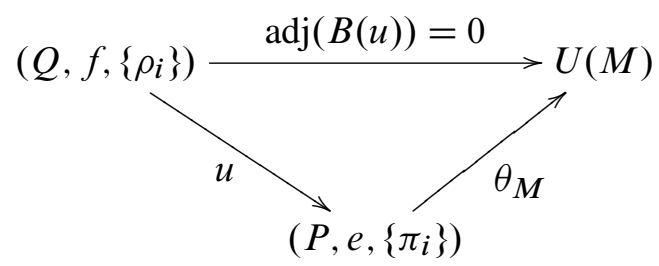

Since $Q$ is f.g. projective there exists $T \in I$ such that

$$
v=s\langle T\rangle:\left(P, e,\left\{\pi_{i}\right\}\right) \rightarrow\left(P\langle T\rangle, e\langle T\rangle,\left\{\pi_{i}\langle T\rangle\right\}\right)
$$

is a $B$-isomorphism in $\operatorname{Sei}(A)$ (ie $v \in \Xi$ ) with $v u=0$.

(iv) Immediate from (i)-(iii).

This completes the proof of Theorem B of the Introduction.

\section{Primitive Seifert modules}

This section is devoted to the kernel of the covering functor $B: \operatorname{Sei}(A) \rightarrow \mathcal{F} l k(A)$. Following the terminology of Sheiham [41]:

Definition 4.1 (i) A Seifert $A$-module $\left(P, e,\left\{\pi_{i}\right\}\right)$ is primitive if

$$
B\left(P, e,\left\{\pi_{i}\right\}\right)=0
$$

or equivalently $1-e+e z: P\left[F_{\mu}\right] \rightarrow P\left[F_{\mu}\right]$ is an $A\left[F_{\mu}\right]$-module isomorphism.

(ii) Let $\operatorname{Prim}(A) \subset \mathcal{S e i}(A)$ be the full subcategory with objects the primitive f.g. projective Seifert $A$-modules.

We shall now obtain an intrinsic characterization of the objects in $\mathcal{P} \operatorname{rim}(A)$, generalizing the results for $\mu=1$ recalled below.

Definition 4.2 (Lück and Ranicki [28, Section 5]) A near-projection $(P, e)$ is an $A-$ module $P$ together with an endomorphism $e \in \operatorname{End}_{A}(P)$ such that $e(1-e) \in \operatorname{End}_{A}(P)$ is nilpotent. 
Proposition 4.3 (Bass, Heller and Swan [5], Lück and Ranicki [28])

(i) A linear morphism of induced f.g. projective $A[z]-$ modules

$$
f_{0}+f_{1} z: P[z] \rightarrow Q[z]
$$

is an isomorphism if and only if $f_{0}+f_{1}: P \rightarrow Q$ is an isomorphism and

$$
e=\left(f_{0}+f_{1}\right)^{-1} f_{1}: P \rightarrow P
$$

is nilpotent.

(ii) A linear morphism of induced f.g. projective $A\left[z, z^{-1}\right]$-modules

$$
f_{0}+f_{1} z: P\left[z, z^{-1}\right] \rightarrow Q\left[z, z^{-1}\right]
$$

is an isomorphism if and only if $f_{0}+f_{1}: P \rightarrow Q$ is an isomorphism and

$$
e=\left(f_{0}+f_{1}\right)^{-1} f_{1}: P \rightarrow P
$$

is a near-projection.

(iii) Suppose that $(P, e)$ is a near-projection, or equivalently that

$$
1-e+z e: P\left[z, z^{-1}\right] \rightarrow P\left[z, z^{-1}\right]
$$

is an $A\left[z, z^{-1}\right]$-module automorphism. If $N \geqslant 0$ is so large that $(e(1-e))^{N}=0$ then

$$
e^{N}+(1-e)^{N}: P \rightarrow P
$$

is an $A$-module automorphism, and the endomorphism

$$
e_{\omega}=\left(e^{N}+(1-e)^{N}\right)^{-1} e^{N}: P \rightarrow P
$$

is a projection, with $e_{\omega}\left(1-e_{\omega}\right)=0$. The submodules of $P$

$$
\begin{aligned}
& P^{+}=\left(1-e_{\omega}\right)(P)=(1-e)^{N}(P)=\left\{x \in P \mid(1-e+e z)^{-1} e(x) \in P[z]\right\}, \\
& P^{-}=e_{\omega}(P)=e^{N}(P)=\left\{x \in P \mid(1-e+e z)^{-1}(1-e)(x) \in z^{-1} P\left[z^{-1}\right]\right\}
\end{aligned}
$$

are such that

$$
(P, e)=\left(P^{+}, e^{+}\right) \oplus\left(P^{-}, e^{-}\right)
$$

with $e^{+}: P^{+} \rightarrow P^{+}$and $1-e^{-}: P^{-} \rightarrow P^{-}$nilpotent.

Definition 4.4 A f.g. projective Seifert $A$-module $\left(P, e,\left\{\pi_{i}\right\}\right)$ is strongly nilpotent if the $A\left[F_{\mu}^{+}\right]$-module endomorphism

$$
e z=\sum_{i=1}^{\mu} e \pi_{i} z_{i}: P\left[F_{\mu}^{+}\right] \rightarrow P\left[F_{\mu}^{+}\right]
$$


is nilpotent, ie $(e z)^{N}=0$ for some $N \geqslant 1$.

The condition for strong nilpotence is equivalent to the $A\left[F_{\mu}\right]$-module endomorphism

$$
e z=\sum_{i=1}^{\mu} e \pi_{i} z_{i}: P\left[F_{\mu}\right] \rightarrow P\left[F_{\mu}\right]
$$

being nilpotent.

Expressed as a representation of the complete quiver $Q_{\mu}$, a Seifert module $\left(P, \rho: Q_{\mu} \rightarrow\right.$ End $_{A} P$ ) is strongly nilpotent if and only if there exists $N \geqslant 1$ such that $\rho(p)=0$ for every path $p \in Q_{\mu}$ of length $\geqslant N$.

Proposition 4.5 The following conditions on a f.g. projective Seifert $A$-module $\left(P, e,\left\{\pi_{i}\right\}\right)$ are equivalent:

(i) $\left(P, e,\left\{\pi_{i}\right\}\right)$ is strongly nilpotent,

(ii) the $A\left[F_{\mu}^{+}\right]$-module endomorphism

$$
1-e z: P\left[F_{\mu}^{+}\right] \rightarrow P\left[F_{\mu}^{+}\right]
$$

is an automorphism,

(iii) the $A\left[F_{\mu}^{+}\right]$-module endomorphism

$$
1-e+e z: P\left[F_{\mu}^{+}\right] \rightarrow P\left[F_{\mu}^{+}\right]
$$

is an automorphism.

Proof (i) $\Longrightarrow$ (ii) If $(e z)^{N}=0$ then $1-e z$ has inverse

$$
\begin{aligned}
(1-e z)^{-1}=1+e z+ & (e z)^{2}+\cdots+(e z)^{N-1} \\
& \in \operatorname{Hom}_{A\left[F_{\mu}^{+}\right]}\left(P\left[F_{\mu}^{+}\right], P\left[F_{\mu}^{+}\right]\right)=\operatorname{Hom}_{A}(P, P)\left[F_{\mu}^{+}\right] .
\end{aligned}
$$

(ii) $\Longrightarrow$ (i) The inverse of $1-e z$ is of the form

$$
(1-e z)^{-1}=\sum_{\substack{1 \leqslant i_{1}, i_{2}, \ldots, i_{k} \leqslant \mu \\ n_{1}, n_{2}, \ldots, n_{k} \geqslant 0 \\ n_{1}+n_{2}+\cdots+n_{k}<N}} f_{i_{1} i_{2} \ldots i_{k}} z_{i_{1}}^{n_{1}} z_{i_{2}}^{n_{2}} \ldots z_{i_{k}}^{n_{k}}: P\left[F_{\mu}^{+}\right] \rightarrow P\left[F_{\mu}^{+}\right]
$$

for some $N \geqslant 1$. We have the identity

$$
\begin{aligned}
(1-e z)^{-1}-(1+e z+ & \left.(e z)^{2}+\cdots+(e z)^{N-1}\right)=(1-e z)^{-1}(e z)^{N} \\
& \in \operatorname{Hom}_{A\left[F_{\mu}^{+}\right]}\left(P\left[F_{\mu}^{+}\right], P\left[F_{\mu}^{+}\right]\right)=\operatorname{Hom}_{A}(P, P)\left[F_{\mu}^{+}\right]
\end{aligned}
$$


in which the left hand side is a sum of monomials in $z_{i_{1}} z_{i_{2}}^{n_{2}} \ldots z_{i_{k}}^{n_{k}}$ of degree $n_{1}+$ $n_{2}+\cdots+n_{k}<N$ and the right hand side is a sum of monomials of degree $\geqslant N$. Both sides of the identity are thus 0 ,

$$
(e z)^{N}=0: P\left[F_{\mu}^{+}\right] \rightarrow P\left[F_{\mu}^{+}\right]
$$

and $\left(P, e,\left\{\pi_{i}\right\}\right)$ is strongly nilpotent.

(ii) $\Longleftrightarrow$ (iii) Immediate from the identity

$$
1-e+e z=1-e(1-z): P\left[F_{\mu}^{+}\right] \rightarrow P\left[F_{\mu}^{+}\right]
$$

and the change of variables $z_{i} \mapsto 1-z_{i}$.

Definition 4.6 A $\mu$-component Seifert $A$-module $\left(P, e,\left\{\pi_{i}\right\}\right)$ is a near-projection if it can be expressed as

$$
\left(P, e,\left\{\pi_{i}\right\}\right)=\left(P^{+} \oplus P^{-},\left(\begin{array}{cc}
e^{++} & e^{+-} \\
e^{-+} & e^{--}
\end{array}\right),\left\{\pi_{i}^{+}\right\} \oplus\left\{\pi_{i}^{-}\right\}\right)
$$

and the $2 \mu$-component Seifert $A$-module

$$
\left(P^{\prime}, e^{\prime}, \pi^{\prime}\right)=\left(P^{+} \oplus P^{-},\left(\begin{array}{cc}
e^{++} & -e^{+-} \\
e^{-+} & 1-e^{--}
\end{array}\right),\left\{\pi_{i}^{+}\right\} \oplus\left\{\pi_{i}^{-}\right\}\right)
$$

is strongly nilpotent.

Lemma 4.7 For a near-projection $\left(P, e,\left\{\pi_{i}\right\}\right)$ the pairs $(P, e),\left(P, e^{\prime}\right)$ are nearprojections.

Proof We have a decomposition $P=P^{+} \oplus P^{-}$with respect to which $e^{\prime}$ is strongly nilpotent. Now

$$
\begin{aligned}
& e(1-e)=\left(\begin{array}{cc}
e^{++} & e^{+-} \\
e^{-+} & e^{--}
\end{array}\right)\left(\begin{array}{cc}
1-e^{++} & -e^{+-} \\
-e^{-+} & 1-e^{--}
\end{array}\right) \\
& =\left(\begin{array}{cc}
e^{++}-\left(e^{++}\right)^{2}-e^{+-} e^{-+} & -e^{++} e^{+-}+e^{+-}\left(1-e^{--}\right) \\
e^{-+}-e^{-+} e^{++}-e^{--} e^{-+} & -e^{-+} e^{+-}+e^{--}\left(1-e^{--}\right)
\end{array}\right) \\
& =\left(\begin{array}{cc}
e^{++}-\left(e^{++}\right)^{2}-e^{+-} e^{-+} & -e^{++} e^{+-}+e^{+-}\left(1-e^{--}\right) \\
e^{-+}-e^{-+} e^{++}-e^{--} e^{-+} & -e^{-+} e^{+-}+\left(1-e^{--}\right)-\left(1-e^{--}\right)^{2}
\end{array}\right) .
\end{aligned}
$$

The matrix

$$
e^{\prime}=\left(\begin{array}{c|c}
e^{++} & -e^{+-} \\
\hline e^{-+} & 1-e^{--}
\end{array}\right)
$$


denotes a strongly nilpotent representation of the complete quiver $Q_{2 \mu}$ on $2 \mu$ vertices. In the following illustration $\mu=1$ :

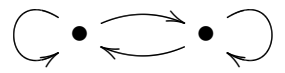

Now each entry in the $2 \mu \times 2 \mu$ matrix $e(1-e)$ above is (the image of) a linear combination of paths of length at least one in the quiver. Hence each entry of $(e(1-e))^{N}$ is the image of a sum of paths of length at least $N$. It follows that $(e(1-e))^{N}=0$ for some $N \geqslant 1$.

The pair $\left(P, e^{\prime}\right)$ is a near-projection since $e^{\prime}: P \rightarrow P$ is nilpotent.

For $\mu=1$ there is no difference between a near-projection $\left(P, e,\left\{\pi_{i}\right\}\right)$ and a nearprojection $(P, e)$. For $\mu \geqslant 2$ a near-projection $\left(P, e,\left\{\pi_{i}\right\}\right)$ has $(P, e)$ a near-projection (Lemma 4.7) but the splitting $(P, e)=\left(P^{+}, e^{+}\right) \oplus\left(P^{-}, e^{-}\right)$given by Proposition 4.3 does not in general extend to a direct sum decomposition of Seifert $A$-modules

$$
\left(P, e,\left\{\pi_{i}\right\}\right)=\left(P^{+}, e^{+},\left\{\pi_{i}^{+}\right\}\right) \oplus\left(P^{-}, e^{-},\left\{\pi_{i}^{-}\right\}\right) .
$$

This is illustrated by the following example.

Example 4.8 Let $A$ be a field, and consider the 2-component Seifert $A$-module $\left(P, e,\left\{\pi_{1}, \pi_{2}\right\}\right)$ given by

$$
P=A^{4}, \quad e=\left(\begin{array}{ll|ll}
0 & 0 & 0 & 0 \\
0 & 1 & 1 & 0 \\
\hline 0 & 0 & 0 & 0 \\
1 & 0 & 0 & 1
\end{array}\right), \pi_{1}=\left(\begin{array}{llll}
1 & 0 & 0 & 0 \\
0 & 1 & 0 & 0 \\
0 & 0 & 0 & 0 \\
0 & 0 & 0 & 0
\end{array}\right), \pi_{2}=\left(\begin{array}{llll}
0 & 0 & 0 & 0 \\
0 & 0 & 0 & 0 \\
0 & 0 & 1 & 0 \\
0 & 0 & 0 & 1
\end{array}\right) .
$$

In this case $e: P \rightarrow P$ is a projection, with $e(1-e)=0$. This f.g. projective Seifert $A$-module has just one submodule

$$
\left(\bar{P}, \bar{e},\left\{\bar{\pi}_{1}, \bar{\pi}_{2}\right\}\right) \subseteq\left(P, e,\left\{\pi_{1}, \pi_{2}\right\}\right)
$$

namely

$$
\bar{P}=e(P)=\left\{(0, x, 0, y) \in P \mid(x, y) \in A^{2}\right\} .
$$

It is not possible to decompose $\left(P, e,\left\{\pi_{1}, \pi_{2}\right\}\right)$ as a direct sum, since $\left(\bar{P}, \bar{e},\left\{\bar{\pi}_{1}, \bar{\pi}_{2}\right\}\right)$ is not a summand. Neither $e$ nor $1-e$ is nilpotent but

$$
1-e+e z=\left(\begin{array}{cccc}
1 & 0 & 0 & 0 \\
0 & z_{1} & z_{2}-1 & 0 \\
0 & 0 & 1 & 0 \\
z_{1}-1 & 0 & 0 & z_{2}
\end{array}\right)=\left(\begin{array}{cccc}
1 & 0 & 0 & 0 \\
0 & 1 & z_{2}-1 & 0 \\
0 & 0 & 1 & 0 \\
z_{1}-1 & 0 & 0 & 1
\end{array}\right)\left(\begin{array}{cccc}
1 & 0 & 0 & 0 \\
0 & z_{1} & 0 & 0 \\
0 & 0 & 1 & 0 \\
0 & 0 & 0 & z_{2}
\end{array}\right)
$$


and

$$
\left(\begin{array}{cccc}
0 & 0 & 0 & 0 \\
0 & 0 & z_{2}-1 & 0 \\
0 & 0 & 0 & 0 \\
z_{1}-1 & 0 & 0 & 0
\end{array}\right)^{2}=0
$$

so $1-e+e z$ is invertible. Moreover, $\left(P, e,\left\{\pi_{1}, \pi_{2}\right\}\right)$ is a near-projection, with $P_{1}^{+}=A \oplus 0 \oplus 0 \oplus 0, P_{1}^{-}=0 \oplus A \oplus 0 \oplus 0, P_{2}^{+}=0 \oplus 0 \oplus A \oplus 0, P_{2}^{-}=0 \oplus 0 \oplus 0 \oplus A$ such that

$$
e^{\prime}=\left(\begin{array}{llll}
0 & 0 & 0 & 0 \\
0 & 0 & 1 & 0 \\
0 & 0 & 0 & 0 \\
1 & 0 & 0 & 0
\end{array}\right): P=P_{1}^{+} \oplus P_{1}^{-} \oplus P_{2}^{+} \oplus P_{2}^{-} \rightarrow P=P_{1}^{+} \oplus P_{1}^{-} \oplus P_{2}^{+} \oplus P_{2}^{-}
$$

is strongly nilpotent.

The main result of this section is:

Theorem 4.9 A f.g. projective Seifert $A$-module $\left(P, e,\left\{\pi_{i}\right\}\right)$ is primitive if and only if it is a near-projection.

Proof Suppose that $\left(P, e,\left\{\pi_{i}\right\}\right)$ is a near-projection, with $e^{\prime}=\left(\begin{array}{cc}e^{++} & -e^{+-} \\ e^{-+} & 1-e^{--}\end{array}\right)$ strongly nilpotent. We have

$$
\begin{aligned}
1-e+e z & =1-e(1-z) \\
& =\left(\begin{array}{cc}
1-e^{++}(1-z) & -e^{+-}(1-z) \\
-e^{-+}(1-z) & 1-e^{--}(1-z)
\end{array}\right) \\
& =\left(\begin{array}{cc}
1-e^{++}(1-z) & e^{+-}\left(1-z^{-1}\right) \\
-e^{-+}(1-z) & 1-\left(1-e^{--}\right)\left(1-z^{-1}\right)
\end{array}\right)\left(\begin{array}{ll}
1 & 0 \\
0 & z
\end{array}\right) \\
& =\left(\left(\begin{array}{ll}
1 & 0 \\
0 & 1
\end{array}\right)-\left(\begin{array}{cc}
e^{++} & -e^{+-} \\
e^{-+} & 1-e^{--}
\end{array}\right)\left(\begin{array}{cc}
1-z & 0 \\
0 & 1-z^{-1}
\end{array}\right)\right)\left(\begin{array}{ll}
1 & 0 \\
0 & z
\end{array}\right):\left(P^{+} \oplus P^{-}\right)\left[F_{\mu}\right] \\
& \longrightarrow\left(P^{+} \oplus P^{-}\right)\left[F_{\mu}\right] .
\end{aligned}
$$

It follows from the strong nilpotence of $e^{\prime}$ that $e^{\prime}\left((1-z) \oplus\left(1-z^{-1}\right)\right)$ is nilpotent, and hence that

$1-e(1-z)=\left(1-e^{\prime}\left((1-z) \oplus\left(1-z^{-1}\right)\right)\right)(1 \oplus z):\left(P^{+} \oplus P^{-}\right)\left[F_{\mu}\right] \rightarrow\left(P^{+} \oplus P^{-}\right)\left[F_{\mu}\right]$ 
is an isomorphism, so that $B\left(P, e,\left\{\pi_{i}\right\}\right)=0$ and $\left(P, e,\left\{\pi_{i}\right\}\right)$ is primitive.

Conversely, suppose that $\left(P, e,\left\{\pi_{i}\right\}\right)$ is a primitive f.g. projective Seifert $A$-module, ie such that the $A\left[F_{\mu}\right]$-module morphism

$$
1-e+e z: P\left[F_{\mu}\right] \rightarrow P\left[F_{\mu}\right]
$$

is an isomorphism. We shall use a variant $\bar{G}_{\mu}$ of the Cayley tree $G_{\mu}$ (Definition 1.3) to prove that $1-e+e z: P\left[F_{\mu}\right] \rightarrow P\left[F_{\mu}\right]$ is a near-projection. Define

$$
\bar{G}_{\mu}^{(0)}=F_{\mu}, \quad \bar{G}_{\mu}^{(1)}=\left\{\left(w, z_{i} w\right) \mid w \in F_{\mu}, i \in\{1,2, \ldots, \mu\}\right\}
$$

so that there is defined a right $F_{\mu}$-action

$$
\bar{G}_{\mu} \times F_{\mu} \rightarrow \bar{G}_{\mu} ;(w, g) \mapsto w g .
$$

For each $i=1,2, \ldots, \mu$ partition $F_{\mu}$ as

$$
F_{\mu}=F_{\mu}^{+, i} \sqcup F_{\mu}^{-, i} \sqcup\{1\}
$$

with $F_{\mu}^{+, i}$ (resp. $F_{\mu}^{-, i}$ ) consisting of the reduced words in $z_{1}, z_{2}, \ldots, z_{\mu}$ which start (resp. do not start) with $z_{i}$. Removing the edge $\left(w, z_{i} w\right)$ disconnects $\bar{G}_{\mu}$, and the complement is a disjoint union of trees

$$
\bar{G}_{\mu}-\left\{\left(w, z_{i} w\right)\right\}=\bar{G}_{\mu}^{+}\left(w, z_{i} w\right) \sqcup \bar{G}_{\mu}^{-}\left(w, z_{i} w\right)
$$

with

$$
\bar{G}_{\mu}^{+}\left(w, z_{i} w\right)^{(0)}=F^{+, i} w, \bar{G}_{\mu}^{-}\left(w, z_{i} w\right)^{(0)}=\left(F^{-, i} \cup\{1\}\right) w .
$$

In the diagram

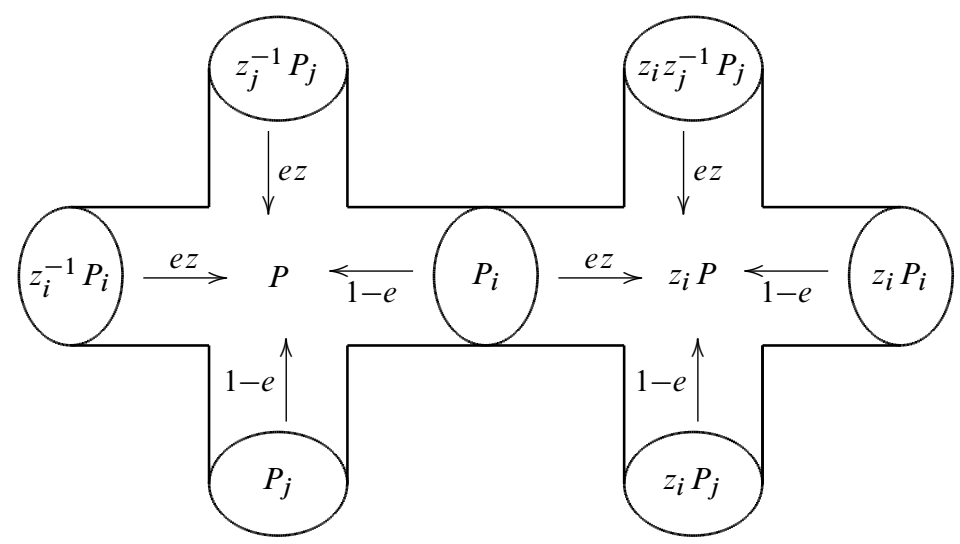

we are placing the components of the range (resp. domain) $P\left[F_{\mu}\right]$ at the vertices (resp. edges) of $\bar{G}_{\mu}$, with the $A$-module $w P$ at $w \in \bar{G}_{\mu}^{(0)}$, and the $A$-module $w P_{i}$ at 
$\left(w, z_{i} w\right) \in \bar{G}_{\mu}^{(1)}$. An element

$$
x \in P\left[F_{\mu}\right]=\sum_{\left(w, z_{i} w\right) \in \bar{G}_{\mu}^{(1)}} w P_{i}
$$

is sent to

$$
(1-e)(x)+e z(x) \in P\left[F_{\mu}\right]=\sum_{w \in \bar{G}_{\mu}^{(0)}} w P,
$$

as indicated by the arrows in the diagram. For $i=1,2, \ldots, \mu$ define the $A$-modules

$$
\begin{aligned}
& P_{i}^{+}=\left\{x \in P_{i} \mid(1-e+e z)^{-1} e z(x) \in \sum_{w \in F_{\mu}^{+, i}} w P\right\}, \\
& P_{i}^{-}=\left\{x \in P_{i} \mid(1-e+e z)^{-1}(1-e)(x) \in \sum_{j \neq i} P_{j} \oplus \sum_{w \in F_{\mu}^{-, i}} w P\right\} .
\end{aligned}
$$

An element $x^{+} \in P_{i}$ belongs to $P_{i}^{+}$if and only if there exist elements $y^{+}(w) \in P$ $\left(w \in F_{\mu}^{+, i}\right)$ such that

$$
e z\left(x^{+}\right)=(1-e+e z)\left(\sum_{w \in F_{\mu}^{+, i}} w y^{+}(w)\right) \in \sum_{w \in F_{\mu}^{+, i}} w P
$$

There is one component $y^{+}(w)$ for each edge in $\bar{G}_{\mu}^{+}\left(1, z_{i}\right)^{(1)}$, and one equation for each vertex in $\bar{G}_{\mu}^{+}\left(1, z_{i}\right)^{(0)}$. Similarly, an element $x^{-} \in P_{i}$ belongs to $P_{i}^{-}$if and only if there exist elements $y_{j} \in P_{j}(j \neq i)$ and $y^{-}(w) \in P\left(w \in F_{\mu}^{-, i}\right)$ such that

$$
\left((1-e)\left(x^{-}\right), 0\right)=(1-e+e z)\left(\sum_{j \neq i} y_{j}+\sum_{w \in F_{\mu}^{-, i}} w y^{-}(w)\right) \in P \oplus \sum_{w \in F_{\mu}^{-, i}} w P . \quad(* *)
$$

There is one component $y_{j}(j \neq i)$ or $y^{-}(w)$ for each edge $\bar{G}_{\mu}^{-}\left(1, z_{i}\right)^{(1)}$, and one equation for each vertex in $\bar{G}_{\mu}^{-}\left(1, z_{i}\right)^{(0)}$. For $i=1,2, \ldots, \mu$ partition

$$
F_{\mu}^{+, i}=F_{\mu}^{++, i} \sqcup F_{\mu}^{-+, i}, \quad F_{\mu}^{-, i}=F_{\mu}^{+-, i} \sqcup F_{\mu}^{--, i}
$$

with $F_{\mu}^{\alpha+, i}$ consisting of the words $w=z_{i_{0}}^{\epsilon_{0}} \ldots z_{i_{k}}^{\epsilon_{k}} \in F_{\mu}$ with $\left(i_{0}, \epsilon_{0}\right)=(i,+), \epsilon_{k}=\alpha$, and $F_{\mu}^{\alpha-, i}$ consisting of the words $w=z_{i_{0}}^{\epsilon_{0}} \ldots z_{i_{k}}^{\epsilon_{k}} \in F_{\mu}$ with $\left(i_{0}, \epsilon_{0}\right) \neq(i,+), \epsilon_{k}=\alpha$. For any $x^{+} \in P_{i}^{+}$and $w \in F_{\mu}^{\alpha+, i}$ we have that $y^{+}(w) \in P_{j}^{\alpha}$, as given by all the terms in (*) involving $\bar{G}^{+}\left(w, z_{i} w\right)$. Similarly, for any $x^{-} \in P_{i}^{-}$and $w \in F_{\mu}^{\alpha-, i}$ we have that $y^{-}(w) \in P_{j}^{\alpha}$, as given by all the terms in $(* *)$ involving $\bar{G}^{-}\left(w, z_{i} w\right)$. 
Regarded as an $A$-module isomorphism $1-e+e z: P\left[F_{\mu}\right] \rightarrow P\left[F_{\mu}\right]$ can be expressed as

$$
\begin{aligned}
1-e+e z= & \\
\left(\begin{array}{ccc}
e z \mid & (1-e+e z) \mid & 0 \\
(1-e) \mid & 0 & (1-e+e z) \mid
\end{array}\right): P_{i} \oplus & \left(\sum_{w \in F_{\mu}^{+, i}} w P\right) \oplus\left(\sum_{j \neq i} P_{j} \oplus \sum_{w \in F_{\mu}^{-, i}} w P\right) \\
& \longrightarrow\left(\sum_{w \in F_{\mu}^{+, i}} w P\right) \oplus\left(P \oplus \sum_{w \in F_{\mu}^{-, i}} w P\right)
\end{aligned}
$$

so that there is induced an $A$-module isomorphism

$$
\begin{aligned}
{\left[\begin{array}{c}
e z \mid \\
(1-e) \mid
\end{array}\right]: P_{i} \rightarrow } & \left(\operatorname{coker}\left((1-e+e z) \mid: \sum_{w \in F_{\mu}^{+, i}} w P \rightarrow \sum_{w \in F_{\mu}^{+, i}} w P\right)\right) \\
& \oplus\left(\operatorname{coker}\left((1-e+e z) \mid: \sum_{j \neq i} P_{j} \oplus \sum_{w \in F_{\mu}^{-, i}} w P \rightarrow P \oplus \sum_{w \in F_{\mu}^{-, i}} w P\right)\right)
\end{aligned}
$$

and

$$
P_{i}=P_{i}^{+} \oplus P_{i}^{-},
$$

with

$$
\begin{aligned}
&(1-e+e z)^{-1} e z\left(P_{i}^{+}\right) \subseteq \sum_{j=1}^{\mu} \sum_{w \in F_{\mu}^{++, i}} w P_{j}^{+} \oplus \sum_{j=1}^{\mu} \sum_{w \in F_{\mu}^{-+, i}} w P_{j}^{-}, \\
&(1-e+e z)^{-1}(1-e)\left(P_{i}^{-}\right) \subseteq \sum_{j=1}^{\mu} \sum_{w \in F_{\mu}^{+-, i}} w P_{j}^{+} \oplus \sum_{j=1}^{\mu} \sum_{w \in F_{\mu}^{--, i}} w P_{j}^{-} .
\end{aligned}
$$

For $\alpha, \beta \in\{ \pm\}$ let

$$
e_{j i}^{\beta \alpha}: P_{i}^{\alpha} \rightarrow P_{j}^{\beta}
$$

be the $A$-module morphisms such that

$$
e=\left(\begin{array}{cc}
e_{j i}^{++} & e_{j i}^{+-} \\
e_{j i}^{-+} & e_{j i}^{--}
\end{array}\right): P=\sum_{i=1}^{\mu}\left(P_{i}^{+} \oplus P_{i}^{-}\right) \rightarrow P=\sum_{j=1}^{\mu}\left(P_{j}^{+} \oplus P_{j}^{-}\right) .
$$

Let

$$
v_{j i}^{\beta \alpha}(w): P_{i}^{\alpha} \rightarrow P_{j}^{\beta}
$$


be the $A$-module morphisms such that

$$
\begin{aligned}
&-(1-e+e z)^{-1} e z \mid=\left(\begin{array}{l}
\sum_{j=1}^{\mu} \sum_{w \in F_{\mu}^{++, i}} w v_{j i}^{++}(w) \\
\sum_{j=1}^{\mu} \sum_{w \in F_{\mu}^{-+, i}} w v_{j i}^{-+}(w)
\end{array}\right): P_{i}^{+} \\
& \longrightarrow \sum_{j=1}^{\mu} \sum_{w \in F_{\mu}^{++, i}} w P_{j}^{+} \oplus \sum_{j=1}^{\mu} \sum_{w \in F_{\mu}^{-+, i}} w P_{j}^{-}, \\
&-(1-e+e z)^{-1}(1-e) \mid=\left(\begin{array}{ll}
\sum_{j=1}^{\mu} \sum_{w \in F_{\mu}^{+-, i}} w v_{j i}^{+-}(w) \\
\sum_{j=1}^{\mu} \sum_{w \in F_{\mu}^{--, i}} w v_{j i}^{--}(w)
\end{array}\right): P_{i}^{-} \\
& \longrightarrow \sum_{j=1}^{\mu} \sum_{w \in F_{\mu}^{+-, i}} w P_{j}^{+} \oplus \sum_{j=1}^{\mu} \sum_{w \in F_{\mu}^{--, i}} w P_{j}^{-} .
\end{aligned}
$$

Composing with $1-e+e z$ gives

(*)

$$
\begin{aligned}
& \begin{array}{r}
-e_{j i}^{++} z_{i}=\sum_{k=1}^{\mu}\left(\sum_{w \in F_{\mu}^{++, i}} w\left(\delta_{j k}-e_{j k}^{++}\right) v_{k i}^{++}(w)+\sum_{w \in F_{\mu}^{-+, i}} w z_{k}\left(e_{j k}^{+-}\right) v_{k i}^{-+}(w)\right): \\
P_{i}^{+} \longrightarrow \sum_{w \in F_{\mu}^{++, i}} P_{j}^{+},
\end{array} \\
& -e_{j i}^{-+} z_{i}=\sum_{k=1}^{\mu}\left(\sum_{w \in F_{\mu}^{++, i}} w e_{j k}^{-+} v_{k i}^{++}(w)+\sum_{w \in F_{\mu}^{-+, i}} w z_{k} e_{j k}^{--} v_{k i}^{-+}(w)\right): \\
& P_{i}^{+} \longrightarrow \sum_{w \in F_{\mu}^{-+, i}} w P_{j}^{-} \\
& -\left(-e_{j i}^{+-}\right)=\sum_{k=1}^{\mu}\left(\sum_{w \in F_{\mu}^{+-, i}} w\left(\delta_{j k}-e_{j k}^{++}\right) v_{k i}^{+-}(w)+\sum_{w \in F_{\mu}^{-+, i}} w z_{k} e_{j k}^{+-} v_{k i}^{--}(w)\right): \\
& P_{i}^{-} \longrightarrow \sum_{w \in F_{\mu}^{+-, i}} w P_{j}^{+} \\
& -\left(\delta_{j i}-e_{j i}^{--}\right)=\sum_{k=1}^{\mu}\left(\sum_{w \in F_{\mu}^{+-, i}} w e_{j k}^{-+} v_{k i}^{+-}(w)+\sum_{w \in F_{\mu}^{--, i}} w z_{k} e_{j k}^{--} v_{k i}^{--}(w)\right): \\
& P_{i}^{-} \longrightarrow \sum_{w \in F_{\mu}^{--, i}} w P_{j}^{-}
\end{aligned}
$$


Comparing the coefficients of $z_{i}$ and 1 gives

$$
\begin{aligned}
&-\left(\begin{array}{c}
e_{j i}^{++} \\
e_{j i}^{-+}
\end{array}\right)=\sum_{k=1}^{\mu}\left(\left(\begin{array}{c}
\delta_{j k}-e_{j k}^{++} \\
-e_{j k}^{-+}
\end{array}\right) v_{k i}^{++}\left(z_{i}\right)+\left(\begin{array}{c}
e_{j k}^{+-} \\
e_{j k}^{-}
\end{array}\right) v_{k i}^{-+}\left(z_{i} z_{k}^{-1}\right)\right) \\
& P_{i}^{+} \rightarrow P_{j}^{+} \oplus P_{j}^{-}, \\
&-\left(\begin{array}{c}
-e_{j i}^{+-} \\
\delta_{j i}-e_{j i}^{--}
\end{array}\right)=\sum_{k=1}^{\mu}\left(\left(\begin{array}{c}
\delta_{j k}-e_{j k}^{++} \\
-e_{j k}^{-+}
\end{array}\right) v_{k i}^{+-}(1)+\left(\begin{array}{c}
e_{j k}^{+-} \\
e_{j k}^{--}
\end{array}\right) v_{k i}^{--}\left(z_{k}^{-1}\right)\right) \\
& P_{i}^{-} \rightarrow P_{j}^{+} \oplus P_{j}^{-} .
\end{aligned}
$$

Writing

$$
\left(\begin{array}{cc}
v^{++} & v^{+-} \\
v^{-+} & v^{--}
\end{array}\right)=\left(\begin{array}{cc}
v_{k i}^{++}\left(z_{i}\right) & v_{k i}^{+-}(1) \\
v_{k i}^{-+}\left(z_{i} z_{k}^{-1}\right) & v_{k i}^{--}\left(z_{k}^{-1}\right)
\end{array}\right): P^{+} \oplus P^{-} \rightarrow P^{+} \oplus P^{-},
$$

we thus have

$$
-\left(\begin{array}{cc}
e^{++} & -e^{+-} \\
e^{-+} & 1-e^{--}
\end{array}\right)=\left(\begin{array}{cc}
1-e^{++} & e^{+-} \\
-e^{-+} & e^{--}
\end{array}\right)\left(\begin{array}{cc}
v^{++} & v^{+-} \\
v^{-+} & v^{--}
\end{array}\right)
$$

Let $Q_{\mu}^{+,-}$be the quiver with $2 \mu$ vertices $(i, \pm)_{1 \leqslant i \leqslant \mu}$ and one edge $\left(i_{0}, \epsilon_{0}\right) \rightarrow\left(i_{1}, \epsilon_{1}\right)$ for each pair of vertices with $\left(i_{0}, \epsilon_{0}\right) \neq\left(i_{1},-\epsilon_{1}\right)$. (The path ring is given by

$$
\begin{aligned}
& Q_{\mu}^{+,-}= \\
& \mathbb{Z}[s] * \mathbb{Z}\left[\pi_{1}^{+},, \ldots, \pi_{\mu}^{+}, \pi_{1}^{-}, \ldots, \pi_{\mu}^{-} \mid \pi_{i}^{\alpha} \pi_{j}^{\beta}=\delta_{\alpha \beta} \delta_{i j} \pi_{i}^{\alpha}, \sum_{i=1}^{\mu}\left(\pi_{i}^{+}+\pi_{i}^{-}\right)=1\right] \\
& /\left\{\pi_{i}^{\alpha} s \pi_{i}^{-\alpha}\right\}
\end{aligned}
$$

where $\pi_{i}^{\alpha} s \pi_{j}^{\beta}((i, \alpha) \neq(j,-\beta))$ corresponds to the unique path of length 1 from $(i, \alpha)$ to $(j, \beta)$.) In the illustration $\mu=2$ :

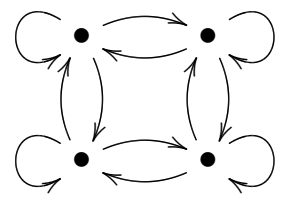

Regard a word $w=z_{i_{0}}^{\epsilon_{0}} z_{i_{1}}^{\epsilon_{1}} \ldots z_{i_{k}}^{\epsilon_{k}} \in F_{\mu}$ as a path of length $|w|=k$ in $Q_{\mu}^{+,-}$

$$
\left(i_{0}, \epsilon_{0}\right) \rightarrow\left(i_{1}, \epsilon_{1}\right) \rightarrow \cdots \rightarrow\left(i_{k}, \epsilon_{k}\right)
$$

and for $k \geqslant 1$ define an $A$-module morphism $v(w): P_{i_{0}}^{\epsilon_{0}} \rightarrow P_{i_{1}}^{\epsilon_{1}}$ as follows. Define

$$
[w]=\left[z_{i_{0}}^{\epsilon_{0}} z_{i_{1}}^{\epsilon_{1}}\right]\left[z_{i_{1}}^{\epsilon_{0}} z_{i_{2}}^{\epsilon_{1}}\right] \ldots\left[z_{i_{k-1}}^{\epsilon_{k-1}} z_{i_{k}}^{\epsilon_{k}}\right] \in F_{\mu}
$$


with

$$
\left[z_{i_{0}}^{\epsilon_{0}} z_{i_{1}}^{\epsilon_{1}}\right]= \begin{cases}z_{i_{0}}^{\epsilon_{0}} & \text { if }\left(\epsilon_{0}, \epsilon_{1}\right)=(+,+) \\ z_{i_{0}}^{\epsilon_{0}} z_{i_{1}}^{\epsilon_{1}} & \text { if }\left(\epsilon_{0}, \epsilon_{1}\right)=(+,-) \\ z_{i_{1}}^{\epsilon_{1}} & \text { if }\left(\epsilon_{0}, \epsilon_{1}\right)=(-,-) \\ 1 & \text { if }\left(\epsilon_{0}, \epsilon_{1}\right)=(-,+) .\end{cases}
$$

For $k=1$ set

$$
v\left(z_{i_{0}}^{\epsilon_{0}} z_{i_{1}}^{\epsilon_{1}}\right)=v_{i_{1} i_{0}}^{\epsilon_{1} \epsilon_{0}}\left(\left[z_{i_{1}}^{\epsilon_{1}} z_{i_{0}}^{\epsilon_{0}}\right]\right)
$$

and for $k \geqslant 2$ set

$$
v\left(z_{i_{0}}^{\epsilon_{0}} z_{i_{1}}^{\epsilon_{1}} \ldots z_{i_{k}}^{\epsilon_{k}}\right)=v\left(z_{i_{k-1}}^{\epsilon_{k-1}} z_{i_{k}}^{\epsilon_{k}}\right) \ldots v\left(z_{i_{1}}^{\epsilon_{1}} z_{i_{2}}^{\epsilon_{2}}\right) v\left(z_{i_{0}}^{\epsilon_{0}} z_{i_{1}}^{\epsilon_{1}}\right) .
$$

The identities

$$
v(w)=v_{i_{k} i_{0}}^{\epsilon_{k} \epsilon_{0}}([w]): P_{i_{0}}^{\epsilon_{0}} \rightarrow P_{i_{k}}^{\epsilon_{k}}
$$

may be verified by induction on $k$, since both sides satisfy the equations $(*)$ and so

$$
\begin{aligned}
-(1-e+e z)^{-1} e z \mid & =\sum_{w \in F_{\mu}^{+, i}} w v(w): P_{i}^{+} \rightarrow \sum_{w \in F_{\mu}^{+, i}} w P, \\
-(1-e+e z)^{-1}(1-e) \mid & =\sum_{j \neq i} v_{j i}^{+-}+\sum_{w \in F_{\mu}^{-, i}} w v(w): P_{i}^{-} \rightarrow \sum_{j \neq i} P_{j} \oplus \sum_{w \in F_{\mu}^{-, i}} w P .
\end{aligned}
$$

For $\alpha, \beta \in\{ \pm\}$ let $F_{\mu}^{\beta \alpha}$ be the set of paths

$$
\left(i_{0}, \epsilon_{0}\right) \rightarrow\left(i_{1}, \epsilon_{1}\right) \rightarrow \cdots \rightarrow\left(i_{k}, \epsilon_{k}\right)
$$

in $Q_{\mu}^{+,-}$with $\epsilon_{0}=\alpha, \epsilon_{k}=\beta$. The $A\left[F_{\mu}\right]$-module endomorphism

$$
v^{\prime}=\left(\begin{array}{ll}
z & 0 \\
0 & 1
\end{array}\right)\left(\begin{array}{ll}
v^{++} & v^{+-} \\
v^{-+} & v^{--}
\end{array}\right)\left(\begin{array}{cc}
1 & 0 \\
0 & z^{-1}
\end{array}\right):\left(P^{+} \oplus P^{-}\right)\left[F_{\mu}\right] \rightarrow\left(P^{+} \oplus P^{-}\right)\left[F_{\mu}\right]
$$

is such that for any $N \geqslant 1$

$$
\begin{aligned}
& \left(v^{\prime}\right)^{N}=\left(\begin{array}{ccc}
\sum_{w \in F_{\mu}^{++},|w|=N} w v^{++}(w) & \sum_{w \in F_{\mu}^{+-},|w|=N} w v^{+-}(w) \\
\sum_{w \in F_{\mu}^{-+},|w|=N} w v^{-+}(w) & \sum_{w \in F_{\mu}^{-},|w|=N} w v^{--}(w)
\end{array}\right): \\
& \left(P^{+} \oplus P^{-}\right)\left[F_{\mu}\right] \rightarrow\left(P^{+} \oplus P^{-}\right)\left[F_{\mu}\right] .
\end{aligned}
$$


If $N \geqslant 1$ is so large that

$$
(1-e+e z)^{-1}=\sum_{w \in F_{\mu},|w|<N} a_{w} w: P\left[F_{\mu}\right] \rightarrow P\left[F_{\mu}\right] \quad\left(a_{w} \in \operatorname{Hom}_{A}\left(P_{i_{0}}, P_{i_{k}}\right)\right)
$$

then for any word $w \in F_{\mu}$ of length $|w|=k>N$

$$
v(w)=0: P_{i_{0}}^{\epsilon_{0}} \rightarrow P_{i_{k}}^{\epsilon_{k}}
$$

The $2 \mu$-component Seifert module

$$
\left(P^{\prime}, v^{\prime}, \pi^{\prime}\right)=\left(P^{+} \oplus P^{-},\left(\begin{array}{ll}
v^{++} & v^{+-} \\
v^{-+} & v^{--}
\end{array}\right),\left\{\pi_{i}^{+} \oplus \pi_{i}^{-}\right\}\right)
$$

is strongly nilpotent, with $\left(v^{\prime} z^{\prime}\right)^{N}=0$, regarding $F_{2 \mu}$ as free group on $2 \mu$ generators $z_{1}^{\prime}, z_{2}^{\prime}, \ldots, z_{2 \mu}^{\prime}$ and letting

$$
z^{\prime}=\left(\begin{array}{cccc}
z_{1}^{\prime} & 0 & \ldots & 0 \\
0 & z_{2}^{\prime} & \ldots & 0 \\
\vdots & \vdots & \ddots & \vdots \\
0 & 0 & \ldots & z_{2 \mu}^{\prime}
\end{array}\right): P^{\prime}\left[F_{2 \mu}\right] \rightarrow P^{\prime}\left[F_{2 \mu}\right]
$$

Define the $2 \mu$-component Seifert module

$$
\left(P^{\prime}, e^{\prime}, \pi^{\prime}\right)=\left(P^{+} \oplus P^{-},\left(\begin{array}{cc}
e^{++} & -e^{+-} \\
e^{-+} & 1-e^{--}
\end{array}\right),\left\{\pi_{i}^{+} \oplus \pi_{i}^{-}\right\}\right)
$$

Applying the augmentation $\epsilon: z_{i} \mapsto 1$ to the $A\left[F_{\mu}\right]$-module morphisms

$$
\begin{array}{r}
-\sum_{w \in F_{\mu}^{+, i}} w v(w): P_{i}^{+}\left[F_{\mu}\right] \stackrel{e z}{\longrightarrow} P\left[F_{\mu}\right] \stackrel{(1-e+e z)^{-1}}{\longrightarrow} P\left[F_{\mu}\right], \\
-\left(\sum_{j \neq i} v_{j i}^{+-}+\sum_{w \in F_{\mu}^{-, i}} w v(w)\right): P_{i}^{-}\left[F_{\mu}\right] \stackrel{1-e}{\longrightarrow} P\left[F_{\mu}\right] \stackrel{(1-e+e z)^{-1}}{\longrightarrow} P\left[F_{\mu}\right]
\end{array}
$$


shows that the components of $e^{\prime}$ are given by linear combinations of paths of length $\geqslant 1$

$$
\begin{aligned}
e^{++} & =-\sum_{w \in F_{\mu}^{++}} v(w): P^{+} \rightarrow P^{+}, \\
e^{-+} & =-\sum_{w \in F_{\mu}^{+-}} v(w): P^{+} \rightarrow P^{-}, \\
-e^{+-} & =-\sum_{w \in F_{\mu}^{-+}} v(w): P^{-} \rightarrow P^{+}, \\
1-e^{--} & =-\sum_{w \in F_{\mu}^{--}} v(w): P^{-} \rightarrow P^{-} .
\end{aligned}
$$

The $A\left[F_{2 \mu}\right]$-module endomorphism $e^{\prime} z^{\prime}: P^{\prime}\left[F_{2 \mu}\right] \rightarrow P^{\prime}\left[F_{2 \mu}\right]$ is nilpotent, with

$$
\left(e^{\prime} z^{\prime}\right)^{N}=0
$$

so that $(P, e, \pi)$ is strongly nilpotent.

This completes the proof of Theorem $\mathrm{C}$ of the Introduction.

\section{Algebraic $K$-theory}

We shall obtain our results on the algebraic $K$-theory of $A\left[F_{\mu}\right]$ and Blanchfield and Seifert modules using the Waldhausen [50] algebraic $K$-theory of categories with cofibrations and weak equivalences, and the noncommutative localization algebraic $K$-theory exact sequence of Neeman and Ranicki [30; 31$]$.

\subsection{The algebraic $K$-theory of exact categories}

The higher algebraic $K$-groups $K_{n}(\mathcal{E})$ of an exact category $\mathcal{E}$ are defined by Quillen [32] to be the homotopy groups of a connective spectrum $K(\mathcal{E})$

$$
\pi_{n}(K(\mathcal{E}))=K_{n}(\mathcal{E}) \quad(n \geqslant 0)
$$

with $K_{0}(\mathcal{E})$ the Grothendieck class group. The idempotent completion $\mathcal{E} \rightarrow \mathcal{P}(\mathcal{E})$ induces an injection $K_{0}(\mathcal{E}) \rightarrow K_{0}\left(\mathcal{P}(\mathcal{E})\right.$ ) and isomorphisms $K_{n}(\mathcal{E}) \rightarrow K_{n}(\mathcal{P}(\mathcal{E})$ ) for $n \geqslant 1$, by the cofinality theorem of Grayson [21]. The lower $K$-groups $K_{n}(\mathcal{E})$ $(n \leqslant-1)$ are defined by Schlichting [38] (following on from the definitions of Karoubi 
and Pedersen-Weibel for the lower $K$-groups of filtered additive categories) to be the lower homotopy groups of a nonconnective spectrum $K \mathcal{P}(\mathcal{E})$ such that

$$
\pi_{n}(K \mathcal{P}(\mathcal{E}))=K_{n}(\mathcal{P}(\mathcal{E})) \quad(n \in \mathbb{Z}),
$$

with $K_{n}(\mathcal{E})=K_{n}(\mathcal{P}(\mathcal{E}))$ for $n \neq 0$.

The algebraic $K$-groups of a ring $R$ are the algebraic $K$-groups of the idempotent complete exact category $\mathcal{E}=\mathcal{P} \operatorname{roj}(R)$ of f.g. projective $R$-modules

$$
K_{n}(R)=K_{n}(\operatorname{Proj}(R)) \quad(n \in \mathbb{Z}),
$$

as defined for $-\infty<n \leqslant 1$ in Bass [4], and for $2 \leqslant n<\infty$ in Quillen [32]. The nonconnective spectrum defined by $K(R)=K \mathcal{P}(\mathcal{P} \operatorname{roj}(R))$ has homotopy groups $\pi_{*}(K(R))=K_{*}(R)$.

A Waldhausen category $(\mathcal{C}, w)$ is a small category $\mathcal{C}$ with cofibrations together with a subcategory $w \subset \mathcal{C}$ of weak equivalences satisfying the axioms of [50]. As usual, there is defined a connective algebraic $K$-theory spectrum

$$
K(\mathcal{C}, w)=\Omega\left|w S_{\bullet} \mathcal{C}\right|
$$

with homotopy groups the algebraic $K$-theory groups

$$
K_{n}(\mathcal{C}, w)=\pi_{n}(K(\mathcal{C}, w)) \quad(n \geqslant 0) .
$$

A functor $F:(\mathcal{C}, w) \rightarrow\left(\mathcal{C}^{\prime}, w^{\prime}\right)$ of Waldhausen categories induces a long exact sequence of algebraic $K$-groups

$$
\cdots \rightarrow K_{n+1}(F) \rightarrow K_{n}(\mathcal{C}, w) \stackrel{F}{\rightarrow} K_{n}\left(\mathcal{C}^{\prime}, w^{\prime}\right) \rightarrow \cdots \rightarrow K_{0}(F) \rightarrow 0
$$

with $K_{n}(F)=\pi_{n}\left(F: K(\mathcal{C}, w) \rightarrow K\left(\mathcal{C}^{\prime}, w^{\prime}\right)\right)(n \geqslant 0)$.

As in Thomason and Trobaugh [45, 1.9] we shall only be considering Waldhausen categories $(\mathcal{C}, w)$ which are 'complicial biWaldhausen', so that in particular $\mathcal{C}$ is a full subcategory of the category of chain complexes in an abelian category $\mathcal{A}$, the cofibrations are chain maps which are split injections in each degree, $w$ contains the quasi-isomorphisms ( $=$ the chain maps inducing isomorphisms in homology), and which in addition are closed under the formation of canonical homotopy pushouts and pullbacks.

The homotopy (or derived) category [45, page 269] of a Waldhausen category $(\mathcal{C}, w)$ is the category of fractions

$$
D(\mathcal{C}, w)=w^{-1} \mathcal{C}
$$


which is a triangulated category under the above hypotheses. The idempotent completion $\mathcal{P} D(\mathcal{C}, w)$ is then also triangulated (Balmer and Schlichting [2]), and the class groups $K_{0}(D(\mathcal{C}, w)), K_{0}(\mathcal{P} D(\mathcal{C}, w))$ are defined, with $K_{0}(D(\mathcal{C}, w))=K_{0}(\mathcal{C}, w)$. Schlichting [38] defined the lower $K$-groups $K_{n}(\mathcal{P} D(\mathcal{C}, w))$ for $n \leqslant-1$ for Waldhausen categories as above, and constructed a nonconnective spectrum $K \mathcal{P}(\mathcal{C}, w)$ with homotopy groups

$$
\pi_{n}(K \mathcal{P}(\mathcal{C}, w))=K \mathcal{P}_{n}(\mathcal{C}, w)= \begin{cases}K_{n}(\mathcal{C}, w) & \text { for } n \geqslant 1 \\ K_{0}(\mathcal{P} D(\mathcal{C}, w)) & \text { for } n=0 \\ K \mathcal{P}_{n}(\mathcal{C}, w) & \text { for } n \leqslant-1\end{cases}
$$

A functor $F:(\mathcal{C}, w) \rightarrow\left(\mathcal{C}^{\prime}, w^{\prime}\right)$ of Waldhausen categories induces a long exact sequence of algebraic $K$-groups

$$
\cdots \rightarrow K \mathcal{P}_{n+1}(F) \rightarrow K \mathcal{P}_{n}(\mathcal{C}, w) \stackrel{F}{\rightarrow} K \mathcal{P}_{n}\left(\mathcal{C}^{\prime}, w^{\prime}\right) \rightarrow K \mathcal{P}_{n}(F) \rightarrow \cdots,
$$

with $K \mathcal{P}_{n}(F)=\pi_{n}\left(F: K \mathcal{P}(\mathcal{C}, w) \rightarrow K \mathcal{P}\left(\mathcal{C}^{\prime}, w^{\prime}\right)\right)(n \in \mathbb{Z})$.

Given an exact category $\mathcal{E}$ let $C^{b}(\mathcal{E})$ be the category of bounded chain complexes in $\mathcal{E}$ and chain maps. An object $C$ in $C^{b}(\mathcal{E})$ is acyclic (in the sense of Keller [24, Chapter 11]) if each differential $d: C_{r} \rightarrow C_{r-1}$ factors as $C_{r} \rightarrow Z_{r} \rightarrow C_{r-1}$ with

$$
0 \rightarrow Z_{r+1} \rightarrow C_{r} \rightarrow Z_{r} \rightarrow 0
$$

exact. A morphism $f: C \rightarrow D$ in $C^{b}(\mathcal{E})$ is a quasi-isomorphism if the mapping cone $\mathcal{C}(f)$ is chain equivalent to an acyclic complex. If $\mathcal{E}$ is fully embedded in an abelian category $\mathcal{A}$ with the embedding closed under extensions and the idempotent completion $\mathcal{P}(\mathcal{E})$ is closed under taking kernels of surjections then a quasi-isomorphism is the same as a chain map inducing isomorphisms in homology in the ambient abelian category $\mathcal{A}$ [45, Appendix A].

Let $\left(\mathcal{C}^{b}(\mathcal{E}), w_{\mathcal{E}}\right)$ be the Waldhausen category with cofibrations the chain maps which are degreewise split injections, and $w_{\mathcal{E}} \subset \mathcal{C}^{b}(\mathcal{E})$ the subcategory of quasi-isomorphisms. The derived category

$$
D^{b}(\mathcal{E})=D\left(\mathcal{C}^{b}(\mathcal{E}), w_{\mathcal{E}}\right)
$$

is the category of bounded chain complexes in $\mathcal{E}$ and fractions of chain homotopy classes of chain maps, with denominators quasi-isomorphisms. As usual, let $K^{b}(\mathcal{E})$ be the category of bounded chain complexes in $\mathcal{E}$ and chain homotopy classes of chain maps, and let $w K_{\mathcal{E}} \subset K^{b}(\mathcal{E})$ be the subcategory of quasi-isomorphisms: the localization

$$
D^{b}(\mathcal{E})=\left(w K_{\mathcal{E}}\right)^{-1} K^{b}(\mathcal{E})
$$


has both a left and a right calculus of fractions. The derived category $D^{b}(\mathcal{E})$ is a triangulated category $[45,1.9 .6]$. Balmer and Schlichting $[2,2.12]$ prove that the idempotent completion of the derived category is the derived category of the idempotent completion

$$
\mathcal{P} D^{b}(\mathcal{E})=D^{b}(\mathcal{P}(\mathcal{E}))
$$

and the algebraic $K$-groups are such that

$$
\begin{cases}K_{n}\left(\mathcal{C}^{b}(\mathcal{E}), w_{\mathcal{E}}\right)=K_{n}(\mathcal{E}) & \text { for } n \geqslant 0(\text { Gillet [20]) } \\ K \mathcal{P}_{n}\left(\mathcal{C}^{b}(\mathcal{E}), w_{\mathcal{E}}\right)=K_{n}(\mathcal{P}(\mathcal{E})) & \text { for } n \in \mathbb{Z}(\text { Schlichting [38]) }\end{cases}
$$

By $[45,1.9 .2]$ the Waldhausen category defined in the same way but with cofibrations the chain maps which are degreewise admissible monomorphisms has the same algebraic $K$-theory.

Definition 5.1 Let $F: \mathcal{E} \rightarrow \mathcal{D}$ be a functor of exact categories.

(i) The algebraic $K$-groups $K \mathcal{P}_{*}(\mathcal{E}, \mathcal{D})$ are

$$
K \mathcal{P}_{n}(\mathcal{E}, \mathcal{D})=K \mathcal{P}_{n}\left(C^{b}(\mathcal{E}, \mathcal{D}), w_{(\mathcal{E}, \mathcal{D})}\right) \quad(n \in \mathbb{Z})
$$

with $\left(\mathcal{C}^{b}(\mathcal{E}, \mathcal{D}), w_{(\mathcal{E}, \mathcal{D})}\right) \subset\left(\mathcal{C}^{b}(\mathcal{E}), w_{\mathcal{E}}\right)$ the Waldhausen subcategory with $\mathcal{C}^{b}(\mathcal{E}, \mathcal{D}) \subset$ $\mathcal{C}^{b}(\mathcal{E})$ the full subcategory with objects the bounded chain complexes $C$ in $\mathcal{E}$ which are chain equivalent in $\mathcal{D}$ to acyclic complexes, and

$$
w_{(\mathcal{E}, \mathcal{D})}=w_{\mathcal{E}} \cap \mathcal{C}^{b}(\mathcal{E}, \mathcal{D}) \subset \mathcal{C}^{b}(\mathcal{E}, \mathcal{D})
$$

the subcategory of the quasi-isomorphisms.

(ii) The algebraic $\Gamma K$-groups of $F$ are

$$
\Gamma K_{n}(F)=K \mathcal{P}_{n}\left(C^{b}(\mathcal{E}), w_{\mathcal{D}}\right) \quad(n \in \mathbb{Z})
$$

with $w_{\mathcal{D}} \subset \mathcal{C}^{b}(\mathcal{E})$ the subcategory with morphisms the chain maps in $\mathcal{E}$ which become quasi-isomorphisms in $\mathcal{D}$, or equivalently such that the mapping cones are in $\mathcal{C}^{b}(\mathcal{E}, \mathcal{D})$.

The groups $\Gamma K_{*}(F)$ are the algebraic $K$-theory analogues of the algebraic $L$-theory groups $\Gamma_{*}(F)$ of Cappell and Shaneson [8].

Theorem 5.2 Let $F: \mathcal{E} \rightarrow \mathcal{D}$ be a functor of exact categories. 
(i) The algebraic $K$-groups fit into a commutative braid of exact sequences

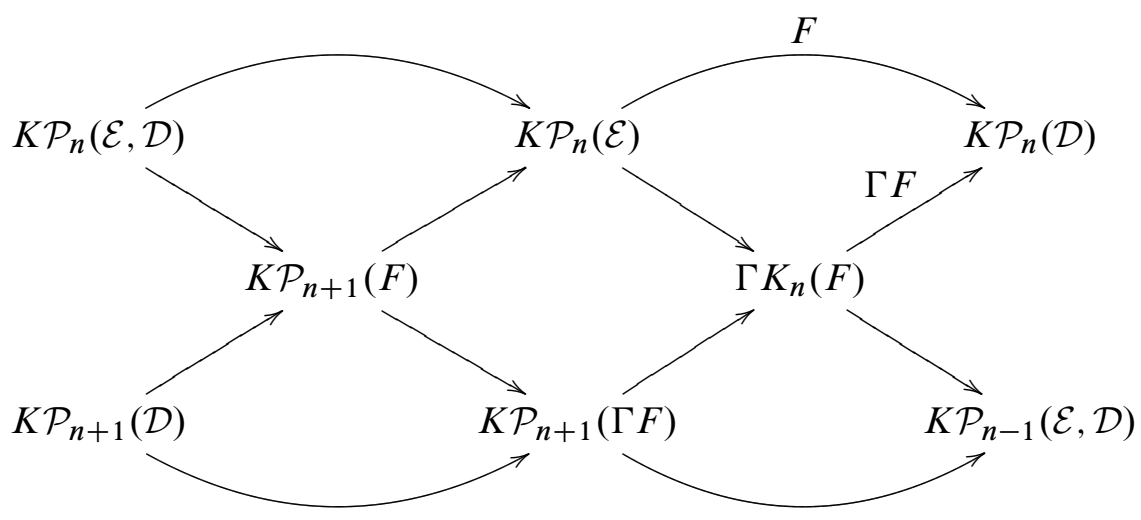

with $\Gamma F:\left(C^{b}(\mathcal{E}), w_{\mathcal{D}}\right) \rightarrow\left(C^{b}(\mathcal{D}), w_{\mathcal{D}}\right)$ induced by $F$.

(ii) If $\Gamma F: \mathcal{P} D\left(\mathcal{C}^{b}(\mathcal{E}), w_{\mathcal{D}}\right) \rightarrow \mathcal{P} D\left(\mathcal{C}^{b}(\mathcal{D}), w_{\mathcal{D}}\right)$ is an equivalence of categories then

$$
K \mathcal{P}_{*}(\Gamma F)=0, K \mathcal{P}_{*+1}(F) \cong K \mathcal{P}_{*}(\mathcal{E}, \mathcal{D}), \Gamma K_{*}(F) \cong K \mathcal{P}_{*}(\mathcal{D})
$$

and the braid of (i) collapses to the exact sequence

$$
\cdots \longrightarrow K \mathcal{P}_{n+1}(\mathcal{D}) \longrightarrow K \mathcal{P}_{n}(\mathcal{E}, \mathcal{D}) \longrightarrow K \mathcal{P}_{n}(\mathcal{E}) \stackrel{F}{\longrightarrow} K \mathcal{P}_{n}(\mathcal{D}) \longrightarrow \cdots
$$

(iii) The hypothesis of (ii) is satisfied if $F: \mathcal{E} \rightarrow \mathcal{D}=\Sigma^{-1} \mathcal{E}$ is the canonical functor to a category of fractions and $\mathcal{D}$ has a calculus of left fractions.

Proof (i) The cases $n \geqslant 0$ are a direct application of the version of the localization theorem of $[50,1.6 .4]$ stated in Theorem 2.3 and Lemma 2.5 of Neeman and Ranicki [31], with

$$
\begin{aligned}
& \mathcal{R}^{c}=D\left(\mathcal{C}^{b}(\mathcal{E}, \mathcal{D}), w_{(\mathcal{E}, \mathcal{D})}\right) \subset \mathcal{S}^{c}=D\left(\mathcal{C}^{b}(\mathcal{E}), w_{\mathcal{E}}\right), \quad \mathcal{S}^{c} / \mathcal{R}^{c} \approx D\left(\mathcal{C}^{b}(\mathcal{E}), w_{\mathcal{D}}\right), \\
& \mathbf{R}=\left(\mathcal{C}^{b}(\mathcal{E}, \mathcal{D}), w_{(\mathcal{E}, \mathcal{D})}\right), \quad \mathbf{S}=\left(\mathcal{C}^{b}(\mathcal{E}), w_{\mathcal{E}}\right), \quad \mathbf{T}=\mathbf{S}_{\mathbf{R}}=\left(\mathcal{C}^{b}(\mathcal{E}), w_{\mathcal{D}}\right)
\end{aligned}
$$

giving a fibration sequence of connective spectra

$$
K\left(\mathcal{C}^{b}(\mathcal{E}, \mathcal{D}), w_{(\mathcal{E}, \mathcal{D})}\right) \rightarrow K\left(\mathcal{C}^{b}(\mathcal{E}), w_{\mathcal{E}}\right) \rightarrow K\left(\mathcal{C}^{b}(\mathcal{E}), w_{\mathcal{D}}\right)
$$

The cases $n<0$ follow from Theorems 2.4, 3.7 of [31] and Schlichting [38, Theorems $1,9]$, which give a fibration sequence of nonconnective spectra

$$
K \mathcal{P}\left(\mathcal{C}^{b}(\mathcal{E}, \mathcal{D}), w_{(\mathcal{E}, \mathcal{D})}\right) \rightarrow K \mathcal{P}\left(\mathcal{C}^{b}(\mathcal{E}), w_{\mathcal{E}}\right) \rightarrow K \mathcal{P}\left(\mathcal{C}^{b}(\mathcal{E}), w_{\mathcal{D}}\right)
$$

(ii) This is a direct application of the Approximation Theorem of Waldhausen [50, Theorem 1.6.7]: if $F:(\mathcal{C}, w) \rightarrow\left(\mathcal{C}^{\prime}, w^{\prime}\right)$ is a functor which induces an equivalence of 
the homotopy categories $F: D(\mathcal{C}, w) \rightarrow D\left(\mathcal{C}^{\prime}, w^{\prime}\right)$ then $F: K(\mathcal{C}, w) \rightarrow K\left(\mathcal{C}^{\prime}, w^{\prime}\right)$ is a homotopy equivalence inducing isomorphisms $F: K_{*}(\mathcal{C}, w) \cong K_{*}\left(\mathcal{C}^{\prime}, w^{\prime}\right)$. Similarly, if $F: \mathcal{P} D(\mathcal{C}, w) \rightarrow \mathcal{P} D\left(\mathcal{C}^{\prime}, w^{\prime}\right)$ is an equivalence there are induced isomorphisms $F: K \mathcal{P}_{*}(\mathcal{C}, w) \cong K \mathcal{P}_{*}\left(\mathcal{C}^{\prime}, w^{\prime}\right)([38])$.

(iii) Every object $D$ in $\mathcal{C}^{b}(\mathcal{D})$ is chain equivalent to $F(E)$ for an object $E$ in $\mathcal{C}^{b}(\mathcal{E})$, and the functors $F: C^{b}(\mathcal{E}) \rightarrow C^{b}(\mathcal{D}), F: D\left(\mathcal{C}^{b}(\mathcal{E}), w_{\mathcal{D}}\right) \rightarrow D\left(\mathcal{C}^{b}(\mathcal{D}), w_{\mathcal{D}}\right)$ are localizations.

Definition 5.3 (i) Write the algebraic $K$-groups of the exact categories $\operatorname{Prim}(A)$, $\operatorname{Sei}(A), \mathcal{B} \operatorname{la}(A), \mathcal{F} l k(A)$ as

$$
\begin{aligned}
& \operatorname{Prim}_{*}(A)=K_{*}(\mathcal{P} \operatorname{rim}(A)), \operatorname{Sei}_{*}(A)=K_{*}(\mathcal{S e i}(A)), \\
& \operatorname{Bla}_{*}(A)=K_{*}(\mathcal{B l a}(A)), \operatorname{Flk}_{*}(A)=K_{*}(\mathcal{F} l k(A)) .
\end{aligned}
$$

(ii) Write the algebraic $K$-groups of the idempotent completion of the homotopy category of $\left(\mathcal{C}^{b}(\mathcal{S e i}(A), \mathcal{B l a}(A)), w_{(\mathcal{S e i}(A), \mathcal{B l a}(A)))}\right)$ as

$$
(\operatorname{Sei}, \mathrm{Bla})_{*}(A)=K \mathcal{P}_{*}\left(\mathcal{C}^{b}(\operatorname{Sei}(A), \mathcal{B l a}(A)), w_{(\operatorname{Sei}(A), \mathcal{B} l a(A))}\right) .
$$

Proposition 5.4 The covering functor $B: \operatorname{Sei}(A) \rightarrow \mathcal{B l a}(A)$ induces morphisms $B: \operatorname{Sei}_{*}(A) \rightarrow \operatorname{Bla}_{*}(A)$ which fit into a long exact sequence

$$
\cdots \longrightarrow(\text { Sei, Bla })_{n}(A) \longrightarrow \operatorname{Sei}_{n}(A) \stackrel{B}{\longrightarrow} \operatorname{Bla}_{n}(A) \longrightarrow(\text { Sei, Bla })_{n-1}(A) \longrightarrow \cdots
$$

with

$$
\operatorname{im}\left(B: \operatorname{Sei}_{0}(A) \rightarrow \operatorname{Bla}_{0}(A)\right)=\operatorname{Flk}_{0}(A) \subseteq \operatorname{Bla}_{0}(A)
$$

Proof Apply Theorem 5.2 (iii) with

$$
F: \mathcal{E}=\operatorname{Sei}(A) \rightarrow \mathcal{D}=\Xi^{-1} \mathcal{S e i}(A) \approx \mathcal{F} l k(A),
$$

noting that $\operatorname{Sei}(A)$ is idempotent complete (Proposition 3.10 (i)), that $\Xi^{-1} \mathcal{S e i}(A) \approx$ $\mathcal{F} l k(A)$ has a left calculus of fractions by Theorem 3.17, and that $\operatorname{Bla}(A) \approx \mathcal{P}(\mathcal{F} l k(A))$ (Proposition 3.10(ii)).

In the next section it will be shown that the functor

$$
\operatorname{Prim}(A) \rightarrow \mathcal{C}^{b}(\operatorname{Sei}(A), \mathcal{B l a}(A)) ;\left(P, e,\left\{\pi_{i}\right\}\right) \mapsto\left(\cdots \rightarrow 0 \rightarrow\left(P, e,\left\{\pi_{i}\right\}\right)\right)
$$

induces isomorphisms of algebraic $K$-groups $\operatorname{Prim}_{*}(A) \cong(\operatorname{Sei}, \mathrm{Bla})_{*}(A)$. 


\subsection{The algebraic $K$-theory of noncommutative localizations}

Given a ring $R$ let $\mathcal{M o d}(R)$ be the abelian category of $R$-modules, so that $\operatorname{Proj}(R) \subset$ $\operatorname{Mod}(R)$ is an exact subcategory. Write the Waldhausen category of $\operatorname{Proj}(R)$ as

$$
\left(\mathcal{C}^{b}(R), w_{R}\right)=\left(\mathcal{C}^{b}(\mathcal{P} \operatorname{roj}(R)), w_{\mathcal{P} r o j(R)}\right) .
$$

An object in $\mathcal{C}^{b}(R)$ is a bounded chain complex $C$ of f.g. projective $R$-modules; $C$ is acyclic if and only if $H_{*}(C)=0$. A morphism $f: C \rightarrow D$ in $\mathcal{C}^{b}(R)$ is a chain map; $f$ is in $w_{R}$ if and only if $f_{*}: H_{*}(C) \rightarrow H_{*}(D)$ is an isomorphism. The algebraic $K$-groups of $R$ are given by

$$
K_{*}(R)=K_{*}(\mathcal{P} \operatorname{roj}(R))=K \mathcal{P}_{*}\left(\mathcal{C}^{b}(R), w_{R}\right) .
$$

A ring morphism $\mathcal{F}: R \rightarrow S$ induces a functor of abelian categories

$$
\mathcal{F}=S \otimes_{R}-: \operatorname{Mod}(R) \rightarrow \operatorname{Mod}(S) ; P \mapsto S \otimes_{R} P
$$

which restricts to an exact functor $F: \mathcal{P} \operatorname{roj}(R) \rightarrow \mathcal{P} \operatorname{roj}(S)$. There is also induced a functor of Waldhausen categories

$$
\mathcal{F}:\left(\mathcal{C}^{b}(R), w_{R}\right) \rightarrow\left(\mathcal{C}^{b}(S), w_{S}\right) ; C \mapsto S \otimes_{R} C .
$$

The relative homotopy groups of $\mathcal{F}: K(R) \rightarrow K(S)$ are the relative $K$-groups $K_{*}(\mathcal{F})$ in the long exact sequence

$$
\cdots \longrightarrow K_{n}(R) \stackrel{\mathcal{F}}{\longrightarrow} K_{n}(S) \longrightarrow K_{n}(\mathcal{F}) \longrightarrow K_{n-1}(R) \longrightarrow \cdots .
$$

Let $R$ be a ring, and let $\Sigma$ be a set of morphisms of f.g. projective $R$-modules. A ring morphism $R \rightarrow T$ is $\Sigma$-inverting if each $(s: P \rightarrow Q) \in \Sigma$ induces a $T$-module isomorphism $1 \otimes s: T \otimes_{R} P \rightarrow T \otimes_{R} Q$. By Cohn [10] there exists a universal $\Sigma$-inverting localization ring morphism

$$
\mathcal{F}: R \rightarrow S=\Sigma^{-1} R
$$

such that any $\Sigma$-inverting ring morphism $R \rightarrow T$ has a unique factorization

$$
R \stackrel{\mathcal{F}}{\longrightarrow} S \longrightarrow T \text {. }
$$

The category of fractions $\Sigma^{-1} \mathcal{P} \operatorname{roj}(R)$ is equivalent to the full subcategory

$$
\operatorname{Proj}_{R}(S) \subseteq \mathcal{P} \operatorname{roj}(S)
$$

with objects isomorphic to the f.g. projective $S$-modules $\Sigma^{-1} P=S \otimes_{R} P$ induced from f.g. projective $R$-modules $P$, and $\mathcal{P} r o j(S)=\mathcal{P}\left(\mathcal{P r o j}_{R}(S)\right)$ is the idempotent completion. 
Definition 5.5 (i) For any ring morphism $\mathcal{F}: R \rightarrow S$ write the Waldhausen categories defined in Definition 5.1 as

$$
\begin{aligned}
\left(\mathcal{C}^{b}(\mathcal{P r o j}(R), \mathcal{P} \operatorname{Proj}(S)), w_{(\mathcal{P r o j}(R), \mathcal{P} r o j(S))}\right) & =\left(\mathcal{C}^{b}(R, S), w_{(R, S)}\right), \\
\left(\mathcal{C}^{b}(\mathcal{P} r o j(R)), w_{S}\right) & =\left(\mathcal{C}^{b}(R), w_{S}\right)
\end{aligned}
$$

with corresponding nonconnective algebraic $K$-theory spectra

$$
K \mathcal{P}\left(\mathcal{C}^{b}(R, S), w_{(R, S)}\right)=K(R, S), K \mathcal{P}\left(\mathcal{C}^{b}(R), w_{S}\right)=\Gamma K(\mathcal{F})
$$

and algebraic $K$-groups $K_{*}(R, S), \Gamma K_{*}(\mathcal{F})$. An object in $\mathcal{C}^{b}(R, S)$ is a bounded chain complex $C$ of f.g. projective $R$-modules such that $H_{*}\left(S \otimes_{R} C\right)=0$. A morphism $f: C \rightarrow D$ in $\mathcal{C}^{b}(R, S)$ is a chain map; $f$ is in $w_{(R, S)}$ if and only if $f_{*}: H_{*}(C) \rightarrow H_{*}(D)$ is an isomorphism. A morphism $f: C \rightarrow D$ in $\mathcal{C}^{b}(R)$ is in $w_{S}$ if and only if $1 \otimes f: H_{*}\left(S \otimes_{R} C\right) \rightarrow H_{*}\left(S \otimes_{R} D\right)$ is an isomorphism.

(ii) For an injective universal localization $\mathcal{F}: R \rightarrow S=\Sigma^{-1} R$ let $H(R, \Sigma)$ be the exact category of h.d. $1 \Sigma$-torsion $R$-modules, ie the cokernels of injective morphisms $s: P \rightarrow Q$ of f.g. projective $R$-modules which induce an $S$-module isomorphism $1 \otimes s: S \otimes_{R} P \rightarrow S \otimes_{R} Q$ (eg if $s \in \Sigma$ ).

(iii) (Neeman and Ranicki [30; 31]) A universal localization $\mathcal{F}: R \rightarrow S=\Sigma^{-1} R$ is stably flat if

$$
\operatorname{Tor}_{i}^{R}(S, S)=0 \quad(i \geqslant 1) .
$$

In particular, a universal localization $\mathcal{F}: R \rightarrow S$ is stably flat if $S$ has flat dimension $\leqslant 1$ as an $R$-module, ie if there exists a 1 -dimensional flat $R$-module resolution

$$
0 \rightarrow F_{1} \rightarrow F_{0} \rightarrow S \rightarrow 0 .
$$

Proposition 5.6 (i) For any ring morphism $\mathcal{F}: R \rightarrow S$ the functor

$$
\Gamma \mathcal{F}:\left(\mathcal{C}^{b}(R), w_{S}\right) \rightarrow\left(\mathcal{C}^{b}(S), w_{S}\right) ; C \mapsto S \otimes_{R} C
$$

induces morphisms of algebraic $K$-groups $\Gamma \mathcal{F}: \Gamma K_{*}(\mathcal{F}) \rightarrow K_{*}(S)$ which fit into a commutative braid of exact sequences

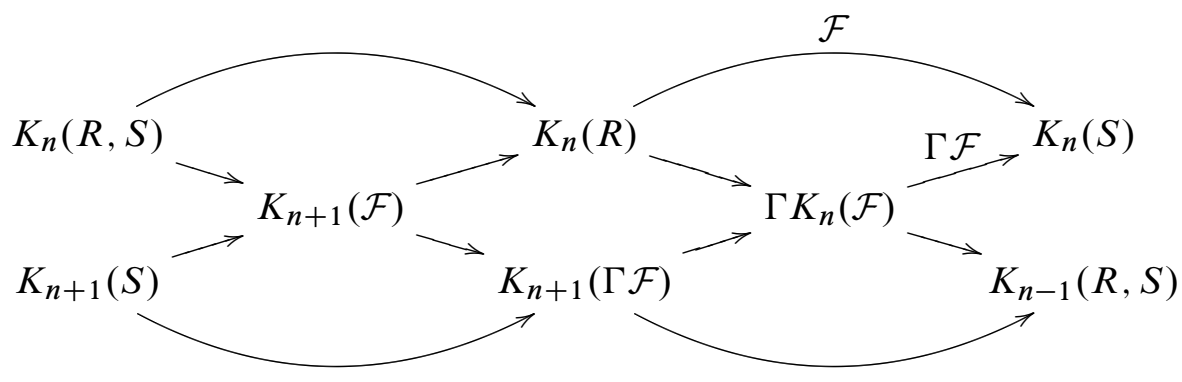


(ii) For any universal localization $\mathcal{F}: R \rightarrow S=\Sigma^{-1} R$

$$
\Gamma K_{n}(\mathcal{F})=K_{n}(S), K_{n}(\mathcal{F})=K_{n-1}(R, S), K_{n}(\Gamma \mathcal{F})=0 \quad(n \leqslant 1) .
$$

(iii) For a stably flat universal localization $\mathcal{F}: R \rightarrow S=\Sigma^{-1} R$

$$
\Gamma K_{*}(\mathcal{F})=K_{*}(S), K_{*+1}(\mathcal{F})=K_{*}(R, S), K_{*}(\Gamma \mathcal{F})=0,
$$

and there is induced a localization exact sequence in the algebraic $K$-groups

$$
\cdots \longrightarrow K_{n}(R, S) \longrightarrow K_{n}(R) \stackrel{\mathcal{F}}{\longrightarrow} K_{n}(S) \longrightarrow K_{n-1}(R, S) \longrightarrow \cdots .
$$

(iv) For an injective universal localization $\mathcal{F}: R \rightarrow S=\Sigma^{-1} R$ there is defined an equivalence of homotopy categories

$$
D\left(\mathcal{C}^{b}(R, S), w_{(R, S)}\right) \approx D\left(C^{b}(H(R, \Sigma)), w_{H(R, \Sigma)}\right)
$$

inducing isomorphisms

$$
K_{*}(R, S) \cong K_{*}(H(R, \Sigma)) .
$$

(v) For an injective stably flat universal localization $\mathcal{F}: R \rightarrow S=\Sigma^{-1} R$ there is defined a localization exact sequence in the algebraic $K$-groups

$$
\cdots \longrightarrow K_{n}(H(R, \Sigma)) \longrightarrow K_{n}(R) \stackrel{\mathcal{F}}{\rightarrow} K_{n}\left(\Sigma^{-1} R\right) \longrightarrow K_{n-1}(H(R, \Sigma)) \longrightarrow \cdots
$$

Proof (i) Immediate from Theorem 5.2 (i) and (ii) applied to $\mathcal{F}: \mathcal{C}^{b}(R) \rightarrow \mathcal{C}^{b}(S)$.

(ii)-(v) See Neeman and Ranicki [30; 31].

\subsection{Triangular matrix rings}

We refer to Haghany and Varadarajan [23] for the general theory of modules over triangular matrix rings, and to Schofield [39], Ranicki [36] and Sheiham [42] for previous accounts of the universal localization of triangular matrix rings.

Proposition 5.7 Let

$$
A=\left(\begin{array}{cc}
A_{1} & B \\
0 & A_{2}
\end{array}\right)
$$

be the triangular $2 \times 2$ matrix ring defined by rings $A_{1}, A_{2}$ and an $\left(A_{1}, A_{2}\right)$-bimodule $B$. 
(i) An $A$-module $L=\left(L_{1}, L_{2}, \lambda\right)$ is defined by an $A_{1}$-module $L_{1}$, an $A_{2}$-module $L_{2}$ and an $A_{1}$-module morphism $\lambda: B \otimes_{A_{2}} L_{2} \rightarrow L_{1}$. As an additive group $L=$ $L_{1} \oplus L_{2}$, written $\left(\begin{array}{l}L_{1} \\ L_{2}\end{array}\right)$, with

$$
\left(\begin{array}{cc}
A_{1} & B \\
0 & A_{2}
\end{array}\right) \times\left(\begin{array}{l}
L_{1} \\
L_{2}
\end{array}\right) \rightarrow\left(\begin{array}{l}
L_{1} \\
L_{2}
\end{array}\right):\left(\left(\begin{array}{cc}
a_{1} & b \\
0 & a_{2}
\end{array}\right),\left(\begin{array}{l}
x_{1} \\
x_{2}
\end{array}\right)\right) \rightarrow\left(\begin{array}{c}
a_{1} x_{1}+\lambda\left(b \otimes x_{2}\right) \\
a_{2} x_{2}
\end{array}\right) .
$$

(ii) An $A$-module morphism $\left(f_{1}, f_{2}\right):\left(L_{1}, L_{2}, \lambda\right) \rightarrow\left(L_{1}^{\prime}, L_{2}^{\prime}, \lambda^{\prime}\right)$ is defined by an $A_{1}$-module morphism $f_{1}: L_{1} \rightarrow L_{1}^{\prime}$, and an $A_{2}$-module morphism $f_{2}: L_{2} \rightarrow L_{2}^{\prime}$ such that the diagram

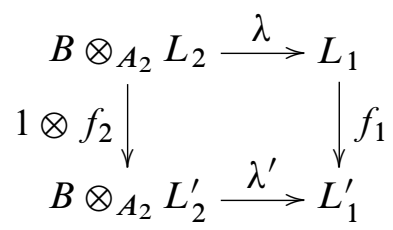

commutes.

(iii) An $A$-module $L=\left(L_{1}, L_{2}, \lambda\right)$ is f.g. projective if and only if $\lambda$ is injective, coker $(\lambda)$ is a f.g. projective $A_{1}$-module, and $L_{2}$ is a f.g. projective $A_{2}$-module.

(iv) The projection

$$
\operatorname{Proj}(A) \rightarrow \operatorname{Proj}\left(A_{1}\right) \times \mathcal{P} \operatorname{roj}\left(A_{2}\right) ; L=\left(L_{1}, L_{2}, \lambda\right) \mapsto\left(\operatorname{coker}(\lambda), L_{2}\right)
$$

induces isomorphisms

$$
K_{*}(A) \cong K_{*}\left(A_{1}\right) \oplus K_{*}\left(A_{2}\right) .
$$

(v) If an $A$-module $L=\left(L_{1}, L_{2}, \lambda\right)$ is h.d. 1 then

(1) the 1-dimensional $A_{1}$-module chain complex

$$
K: \cdots \longrightarrow 0 \longrightarrow B \otimes_{A_{2}} L_{2} \stackrel{\lambda}{\longrightarrow} L_{1}
$$

is such that there exists a quasi-isomorphism (= homology equivalence) $J \rightarrow K$ for a 1-dimensional f.g. projective $A_{1}$-module chain complex $J$, and

(2) $L_{2}$ is an h.d. $1 A_{2}$-module.

If $B$ is a flat right $A_{2}$-module the converse also holds: an $A$-module $L$ is h.d. 1 if and only if conditions 1. and 2. are satisfied.

(vi) The columns of $A$ are f.g. projective $A$-modules

$$
S_{1}=\left(A_{1}, 0,0\right), S_{2}=\left(B, A_{2}, 1\right)
$$


with

$$
S_{1} \oplus S_{2}=A, \operatorname{End}\left(S_{1}\right)=A_{1}, \operatorname{End}\left(S_{2}\right)=A_{2} .
$$

The universal localization of $A$ inverting a non-empty subset $\Sigma \subseteq \operatorname{Hom}_{A}\left(S_{1}, S_{2}\right)$ is a morphism of $2 \times 2$ matrix rings

$$
A=\left(\begin{array}{cc}
A_{1} & B \\
0 & A_{2}
\end{array}\right) \rightarrow \Sigma^{-1} A=M_{2}(C)=\left(\begin{array}{ll}
C & C \\
C & C
\end{array}\right)
$$

with $C$ the endomorphism ring of the induced f.g. projective $\Sigma^{-1} A$-module

$$
\Sigma^{-1} S_{1} \cong \Sigma^{-1} S_{2}
$$

The composite of the functor

$$
\Sigma^{-1}: \operatorname{Mod}(A) \rightarrow \mathcal{M o d}\left(\Sigma^{-1} A\right) ; P \mapsto \Sigma^{-1} P=\Sigma^{-1} A \otimes_{A} P
$$

and the Morita equivalence of categories

$$
\begin{aligned}
(C C) \otimes_{\Sigma^{-1} A}-: \operatorname{Mod}\left(\Sigma^{-1} A\right) & \stackrel{\simeq}{ } \operatorname{Mod}(C) \\
L=\left(L_{1}, L_{2}, \lambda\right) & \longmapsto(C C) \otimes_{\Sigma^{-1} A} L
\end{aligned}
$$

is the assembly functor

$$
\begin{aligned}
\operatorname{Mod}(A) & \longrightarrow \operatorname{Mod}(C) ; \\
L=\left(L_{1}, L_{2}, \lambda\right) & \longmapsto(C C) \otimes_{\Sigma^{-1} A} \Sigma^{-1} L=(C C) \otimes_{A} L \\
& =\operatorname{coker}\left(\left(\begin{array}{c}
1 \otimes \lambda \\
\kappa \otimes 1
\end{array}\right): C \otimes_{A_{1}} B \otimes_{A_{2}} L_{2} \rightarrow C \otimes_{A_{1}} L_{1} \oplus C \otimes_{A_{2}} L_{2}\right)
\end{aligned}
$$

with

$$
\kappa: C \otimes_{A_{1}} B \rightarrow C ; x \otimes y \mapsto x y
$$

the $\left(C, A_{2}\right)$-bimodule morphism defined by multiplication in $C$, using the $A_{1}$-module morphism $B \rightarrow C$. The assembly functor $\operatorname{Proj}(A) \rightarrow \mathcal{P} \operatorname{roj}(C)$ induces the morphisms

$$
\Sigma^{-1}: K_{*}(A)=K_{*}\left(A_{1}\right) \oplus K_{*}\left(A_{2}\right) \rightarrow K_{*}\left(\Sigma^{-1} A\right)=K_{*}(C) .
$$

(vii) If $B$ and $C$ are flat $A_{1}$-modules and $C$ is a flat $A_{2}$-module then the $A$-module $\left(\begin{array}{l}C \\ C\end{array}\right)$ has a 1 -dimensional flat $A$-module resolution

$$
0 \rightarrow\left(\begin{array}{c}
B \\
0
\end{array}\right) \otimes_{A_{2}} C \rightarrow\left(\begin{array}{c}
A_{1} \\
0
\end{array}\right) \otimes_{A_{1}} C \oplus\left(\begin{array}{c}
B \\
A_{2}
\end{array}\right) \otimes_{A_{2}} C \rightarrow\left(\begin{array}{l}
C \\
C
\end{array}\right) \rightarrow 0
$$


so that $\Sigma^{-1} A=(\underset{C}{C}) \oplus(\underset{C}{C})$ is stably flat. An h.d. $1 A$-module $L=\left(L_{1}, L_{2}, \lambda\right)$ is $\Sigma$-torsion if and only if the $C$-module morphism

$$
\left(\begin{array}{c}
1 \otimes \lambda \\
\kappa \otimes 1
\end{array}\right): C \otimes_{A_{1}} B \otimes_{A_{2}} L_{2} \rightarrow C \otimes_{A_{1}} L_{1} \oplus C \otimes_{A_{2}} L_{2}
$$

is an isomorphism.

Proof (i) and (ii) Standard.

(iii) For any $A$-module $L=\left(L_{1}, L_{2}, \lambda\right)$ there is defined an exact sequence

$$
0 \rightarrow(\operatorname{ker}(\lambda), 0,0) \rightarrow\left(B \otimes_{A_{2}} L_{2}, L_{2}, 1\right) \stackrel{(\lambda, 1)}{\longrightarrow}\left(L_{1}, L_{2}, \lambda\right) \rightarrow(\operatorname{coker}(\lambda), 0,0) \rightarrow 0 .
$$

Now $\left(A_{1}, 0,0\right)=\left(\begin{array}{c}A_{1} \\ 0\end{array}\right)$ and $\left(B, A_{2}, 1\right)=\left(\begin{array}{c}B \\ A_{2}\end{array}\right)$ are f.g. projective $A$-modules, since

$$
\left(A_{1}, 0,0\right) \oplus\left(B, A_{2}, 1\right)=\left(A_{1} \oplus B, A_{2},\left(\begin{array}{l}
0 \\
1
\end{array}\right)\right)=A .
$$

If $\operatorname{ker}(\lambda)=0$ and $\operatorname{coker}(\lambda)$ is a f.g. projective $A_{1}$-module then $(\operatorname{coker}(\lambda), 0,0)=$ $\left(A_{1}, 0,0\right) \otimes_{A_{1}} \operatorname{coker}(\lambda)$ is a f.g. projective $A$-module. If $L_{2}$ is a f.g. projective $A_{2}$-module then

$$
\left(B \otimes_{A_{2}} L_{2}, L_{2}, 1\right)=\left(\begin{array}{c}
B \\
A_{2}
\end{array}\right) \otimes_{A_{2}} L_{2}
$$

is a f.g. projective $A$-module. Thus if these two conditions are satisfied then the exact sequence splits and $L$ is a f.g. projective $A$-module.

Conversely, suppose that $\left(L_{1}, L_{2}, \lambda\right)$ is a f.g. projective $A$-module, so that there exists an $A$-module $\left(L_{1}^{\prime}, L_{2}^{\prime}, \lambda^{\prime}\right)$ with an $A$-module isomorphism

$$
\left(L_{1}, L_{2}, \lambda\right) \oplus\left(L_{1}^{\prime}, L_{2}^{\prime}, \lambda^{\prime}\right) \cong\left(\left(A_{1}\right)^{k} \oplus B^{k},\left(A_{2}\right)^{k},\left(\begin{array}{l}
0 \\
1
\end{array}\right)\right)=A^{k}
$$

for some $k \geqslant 0$. It follows from $\operatorname{ker}\left(\lambda \oplus \lambda^{\prime}\right)=0$ that $\operatorname{ker}(\lambda)=0$, and from $\operatorname{coker}(\lambda \oplus$ $\left.\lambda^{\prime}\right) \cong\left(A_{1}\right)^{k}$ that coker $(\lambda)$ is a f.g. projective $A_{1}$-module. Also, $L_{2} \oplus L_{2}^{\prime} \cong\left(A_{2}\right)^{k}$, so that $L_{2}$ is a f.g. projective $A_{2}$-module.

(iv) The result that the inclusion and projection

$$
i=A_{1} \times A_{2} \rightarrow A, \quad j: A \rightarrow A_{1} \times A_{2}
$$

induce inverse isomorphisms

$$
K_{*}\left(A_{1} \times A_{2}\right)=K_{*}\left(A_{1}\right) \oplus K_{*}\left(A_{2}\right) \underset{j_{*}}{\stackrel{i_{*}}{\gtrless}} K_{*}(A)
$$


was first obtained by Berrick and Keating [6]. Here is a proof using Waldhausen $K$-theory. It is immediate from $j i=1$ that

$$
j_{*} i_{*}=1: K_{*}\left(A_{1} \times A_{2}\right) \rightarrow K_{*}(A) \rightarrow K_{*}\left(A_{1} \times A_{2}\right) .
$$

Every f.g. projective $A$-module $L=\left(L_{1}, L_{2}, \lambda: B \otimes_{A_{2}} L_{2} \rightarrow L_{1}\right)$ fits into a natural short exact sequence of f.g. projective $A$-modules

$$
0 \rightarrow\left(B \otimes_{A_{2}} L_{2}, L_{2}, 1\right) \stackrel{(\lambda, 1)}{\longrightarrow}\left(L_{1}, L_{2}, \lambda\right) \rightarrow(\operatorname{coker}(\lambda), 0) \rightarrow 0 .
$$

The functors

$$
\begin{aligned}
& F_{1}: \operatorname{Proj}(A) \rightarrow \mathcal{P} \operatorname{roj}(A) ; L \mapsto A \otimes_{A_{1}} A_{1} \otimes_{A} L=(\operatorname{coker}(\lambda), 0), \\
& F_{2}: \mathcal{P} \operatorname{roj}(A) \rightarrow \mathcal{P} \operatorname{roj}(A) ; L \mapsto A \otimes_{A_{2}} A_{2} \otimes_{A} L=\left(B \otimes_{A_{2}} L_{2}, L_{2}, 1\right)
\end{aligned}
$$

fit into a cofibration sequence

$$
F_{2} \rightarrow 1_{\mathcal{P} r o j(A)} \rightarrow F_{1}
$$

and are such that

$$
F_{k}: K_{*}(A) \rightarrow K_{*}\left(A_{k}\right) \rightarrow K_{*}(A) \quad(k=1,2) .
$$

Now apply the additivity theorem for Quillen $K$-theory [50, Proposition 1.3.2 (4)] to identify

$$
i_{*} j_{*}=F_{1}+F_{2}=1: K_{*}(A) \rightarrow K_{*}(A),
$$

so that $i_{*}, j_{*}$ are inverse isomorphisms.

(v) If $L=\left(L_{1}, L_{2}, \lambda\right)$ is an h.d. $1 A$-module there exists a 1 -dimensional f.g. projective $A$-module resolution

$$
0 \longrightarrow\left(P_{1}, P_{2}, f\right) \stackrel{\left(h_{1}, h_{2}\right)}{\longrightarrow}\left(Q_{1}, Q_{2}, g\right) \longrightarrow\left(L_{1}, L_{2}, \lambda\right) \longrightarrow 0,
$$

so that coker $(f)$, coker $(g)$ are f.g. projective $A_{1}$-modules and $P_{2}, Q_{2}$ are f.g. projective $A_{2}$-modules. The 1 -dimensional $A_{1}$-module chain complex

$$
K: \cdots \longrightarrow 0 \longrightarrow B \otimes_{A_{2}} L_{2} \stackrel{\lambda}{\longrightarrow} L_{1}
$$

and the 1-dimensional f.g. projective $A_{1}$-module chain complex

$$
J: J_{1}=\operatorname{coker}(f) \stackrel{h_{1}}{\longrightarrow} J_{0}=\operatorname{coker}(g)
$$

are related by a homology equivalence $J \rightarrow K$. Furthermore, $L_{2}=\operatorname{coker}\left(h_{2}\right)$ is an h.d. $1 A_{2}$-module. Thus both conditions 1. and 2. are satisfied. 
Conversely, suppose that $B$ is a flat right $A_{2}$-module and that $L=\left(L_{1}, L_{2}, \lambda\right)$ is an $A$ module such that there exists a homology equivalence $J \rightarrow K$ with $J$ a 1-dimensional f.g. projective $A_{1}$-module chain complex and that $L_{2}$ is an h.d. $1 A_{2}$-module with a 1-dimensional f.g. projective $A_{2}$-module resolution

$$
0 \rightarrow P_{2} \rightarrow Q_{2} \rightarrow L_{2} \rightarrow 0 .
$$

There is induced a short exact sequence of $A_{1}$-modules

$$
0 \rightarrow B \otimes_{A_{2}} P_{2} \rightarrow B \otimes_{A_{2}} Q_{2} \rightarrow B \otimes_{A_{2}} L_{2} \rightarrow 0
$$

and it follows from the 1-dimensional f.g. projective $A$-module resolution of $L$

$$
0 \rightarrow\left(B \otimes_{A_{2}} P_{2}, P_{2}, 1\right) \oplus\left(J_{1}, 0,0\right) \rightarrow\left(B \otimes_{A_{2}} Q_{2}, Q_{2}, 1\right) \oplus\left(J_{0}, 0,0\right) \rightarrow L \rightarrow 0
$$

that $L$ is an h.d. $1 A$-module.

(vi) and (vii) See [36, 2.2].

We shall actually be working with $(\mu+1) \times(\mu+1)$-matrix rings:

Definition 5.8 For any ring $A$ and $\mu \geqslant 1$ define the triangular $(\mu+1) \times(\mu+1)$-matrix ring

$$
T_{\mu}(A)=\left(\begin{array}{ccccc}
A & A \oplus A & A \oplus A & \ldots & A \oplus A \\
0 & A & 0 & \ldots & 0 \\
0 & 0 & A & \ldots & 0 \\
\vdots & \vdots & \vdots & \ddots & \vdots \\
0 & 0 & 0 & \ldots & A
\end{array}\right) .
$$

The ring $T_{\mu}(A)$ is the $A$-coefficient path algebra of the quiver with vertices $0,1, \ldots, \mu$ and two arrows $s_{i}^{+}, s_{i}^{-}: i \rightarrow 0$ for $i=1,2, \ldots, \mu$. A $T_{\mu}(A)$-module $L=\left(L_{i}, f_{i}^{+}, f_{i}^{-}\right)$ consists of $A$-modules $L_{0}, L_{1}, \ldots, L_{\mu}$ and $A$-module morphisms $f_{i}^{+}, f_{i}^{-}: L_{i} \rightarrow L_{0}$ $(1 \leqslant i \leqslant \mu)$.

Let $S_{0}, S_{1}, \ldots, S_{\mu}$ be the $T_{\mu}(A)$-modules defined by the columns of $T_{\mu}(A)$, so that

$$
\begin{aligned}
S_{0} & =(A, 0, \ldots, 0 ; 0, \ldots, 0), \\
S_{i} & =(A \oplus A, 0, \ldots, 0, A, 0, \ldots, 0 ; 0, \ldots, 0, \text { id., } 0, \ldots, 0) \quad(1 \leqslant i \leqslant \mu) .
\end{aligned}
$$

It follows from

$$
S_{0} \oplus S_{1} \oplus \cdots \oplus S_{\mu}=T_{\mu}(A)
$$

that each $S_{i}$ is a f.g. projective $T_{\mu}(A)$-module. Let $\sigma=\left\{s_{i}^{+}, s_{i}^{-}\right\}$be the set of f.g. projective $T_{\mu}(A)$-module morphisms

$$
s_{i}^{+}=((10), 0, \ldots, 0), s_{i}^{-}=((01), 0, \ldots, 0): S_{i} \rightarrow S_{0} \quad(1 \leqslant i \leqslant \mu) .
$$


Proposition 5.9 (i) The universal $\sigma$-inverting localization of $T_{\mu}(A)$ is given by the inclusion

$$
\mathcal{F}: T_{\mu}(A) \rightarrow \sigma^{-1} T_{\mu}(A)=M_{\mu+1}\left(A\left[F_{\mu}\right]\right)
$$

with $M_{\mu+1}\left(A\left[F_{\mu}\right]\right)$ the ring of all $(\mu+1) \times(\mu+1)$-matrices with entries in $A\left[F_{\mu}\right]$. The universal localization $\mathcal{F}$ is both injective and stably flat.

(ii) The composite

$$
\operatorname{Mod}\left(T_{\mu}(A)\right) \stackrel{\mathcal{F}}{\longrightarrow} \operatorname{Mod}\left(M_{\mu+1}\left(A\left[F_{\mu}\right]\right)\right) \stackrel{\text { Morita }}{\approx} \operatorname{Mod}\left(A\left[F_{\mu}\right]\right)
$$

sends a $T_{\mu}(A)$-module $L=\left(L_{i}, f_{i}^{+}, f_{i}^{-}\right)$to the assembly $A\left[F_{\mu}\right]$-module

$$
M=\operatorname{coker}\left(\left(f_{1}^{+} z_{1}-f_{1}^{-} \ldots f_{\mu}^{+} z_{\mu}-f_{\mu}^{-}\right): \bigoplus_{i=1}^{\mu} L_{i}\left[F_{\mu}\right] \rightarrow L_{0}\left[F_{\mu}\right]\right) .
$$

(iii) A $T_{\mu}(A)$-module $L=\left(L_{i}, f_{i}^{+}, f_{i}^{-}\right)$is f.g. projective if and only if $L_{0}, \ldots, L_{\mu}$ are f.g. projective $A$-modules and the $A$-module morphism

$$
\left(\begin{array}{lllllll}
f_{1}^{+} & f_{1}^{-} & f_{2}^{+} & f_{2}^{-} & \ldots & f_{\mu}^{+} & f_{\mu}^{-}
\end{array}\right): \bigoplus_{i=1}^{\mu} L_{i} \oplus L_{i} \rightarrow L_{0}
$$

is a split injection. The projection

$$
\operatorname{Proj}\left(T_{\mu}(A)\right) \rightarrow \prod_{\mu+1} \operatorname{Proj}(A) ;\left(L_{i}, f_{i}^{+}, f_{i}^{-}\right) \mapsto\left(L_{0}, L_{1}, L_{2}, \ldots, L_{\mu}\right)
$$

induces isomorphisms in algebraic $K$-theory

$$
K_{*}\left(T_{\mu}(A)\right) \cong \bigoplus_{\mu+1} K_{*}(A)
$$

(iv) A $T_{\mu}(A)$-module $L=\left(L_{i}, f_{i}^{+}, f_{i}^{-}\right)$is h.d. $1 \sigma$-torsion if and only if $L_{0}, \ldots, L_{\mu}$ are f.g. projective $A$-modules and the $A\left[F_{\mu}\right]$-module morphism

$$
\left(f_{1}^{+} z_{1}-f_{1}^{-} f_{2}^{+} z_{2}-f_{2}^{-} \ldots f_{\mu}^{+} z_{\mu}-f_{\mu}^{-}\right): \bigoplus_{i=1}^{\mu} L_{i}\left[F_{\mu}\right] \rightarrow L_{0}\left[F_{\mu}\right]
$$

is an isomorphism. A f.g. projective Seifert $A$-module $\left(P, e,\left\{\pi_{i}\right\}\right)$ is primitive if and only if $\left(P, P_{i}, f_{i}^{+}, f_{i}^{-}\right)$is an h.d. $1 \sigma$-torsion $T_{\mu}(A)$-module. The functor

$$
\operatorname{Prim}(A) \rightarrow H\left(T_{\mu}(A), \sigma\right) ;\left(P, e,\left\{\pi_{i}\right\}\right) \mapsto\left(P, P_{i}, e \pi_{i},(e-1) \pi_{i}\right)
$$


is an equivalence of exact categories, so that

$$
\operatorname{Prim}_{*}(A)=K_{*}\left(H\left(T_{\mu}(A), \sigma\right)\right) .
$$

The forgetful functor

$$
\begin{aligned}
& \operatorname{Prim}(A) \rightarrow \prod_{2 \mu} \operatorname{Proj}(A) \\
& \left(P^{+} \oplus P^{-},\left(\begin{array}{cc}
e^{++} & e^{+-} \\
e^{-+} & e^{--}
\end{array}\right),\left\{\pi_{i}^{+}\right\} \oplus\left\{\pi_{i}^{-}\right\}\right) \mapsto\left(P_{1}^{+}, P_{1}^{-}, \ldots, P_{\mu}^{+}, P_{\mu}^{-}\right)
\end{aligned}
$$

(defined using Theorem 4.9) is split by

$$
\prod_{2 \mu} \mathcal{P} \operatorname{roj}(A) \rightarrow \mathcal{P} \operatorname{rim}(A) ; \quad\left(P_{1}^{+}, P_{1}^{-}, \ldots, P_{\mu}^{+}, P_{\mu}^{-}\right) \mapsto\left(P^{+} \oplus P^{-}, 0,\left\{\pi_{i}^{+}\right\} \oplus\left\{\pi_{i}^{-}\right\}\right) .
$$

The reduced $K$-groups defined by

$$
\widetilde{\operatorname{Prim}}_{*}(A)=\operatorname{ker}\left(\operatorname{Prim}_{*}(A) \rightarrow \bigoplus_{2 \mu} K_{*}(A)\right)
$$

are such that

$$
K_{*}\left(H\left(T_{\mu}(A), \sigma\right)\right)=\operatorname{Prim}_{*}(A)=\bigoplus_{2 \mu} K_{*}(A) \oplus \widetilde{\operatorname{Prim}_{*}}(A)
$$

Proof The universal localization $\sigma^{-1} T_{\mu}(A)$ is the $(\mu+1) \times(\mu+1)$-matrix ring $M_{\mu+1}(R)$ with $R$ the endomorphism ring of the induced f.g. projective $\sigma^{-1} T_{\mu}(A)-$ module $\sigma^{-1} S_{0}$, and there is defined an isomorphism

$$
A\left[F_{\mu}\right] \rightarrow R ; z_{i} \mapsto s_{i}^{+}\left(s_{i}^{-}\right)^{-1} .
$$

The remaining parts are given by Proposition 5.7, viewing the $(\mu+1) \times(\mu+1)$ matrix ring $T_{\mu}(A)$ as a triangular $2 \times 2$ matrix ring

$$
T_{\mu}(A)=\left(\begin{array}{cc}
A_{1} & B \\
0 & A_{2}
\end{array}\right)
$$

with

$$
A_{1}=A, \quad A_{2}=\left(\begin{array}{cccc}
A & 0 & \ldots & 0 \\
0 & A & \ldots & 0 \\
\vdots & \vdots & \ddots & \vdots \\
0 & 0 & \ldots & A
\end{array}\right), \quad B=(A \oplus A \ldots A \oplus A)
$$


such that

$$
\operatorname{Mod}\left(A_{2}\right)=\prod_{\mu} \operatorname{Mod}(A)
$$

An $A_{2}$-module is just a $\mu$-tuple $\left(L_{1}, L_{2}, \ldots, L_{\mu}\right)$ of $A$-modules. By the $2 \times 2$ theory a $T_{\mu}(A)$-module $L$ just a $(\mu+1)$-tuple $\left(L_{0}, L_{1}, \ldots, L_{\mu}\right)$ of $A$-modules, together with $A$-module morphisms $f_{i}^{+}, f_{i}^{-}: L_{i} \rightarrow L_{0}(1 \leqslant i \leqslant \mu)$. Note that $B$ is a flat right $A_{2}$-module, and that for an h.d. $1 \sigma$-torsion $T_{\mu}(A)$-module $L=\left(L_{i}, f_{i}^{+}, f_{i}^{-}\right)$ each $L_{i}(0 \leqslant i \leqslant \mu)$ is a f.g. projective $A$-module, by the following argument. The necessary and sufficient conditions of Proposition $5.7(\mathrm{v})$ and (vii) for a $T_{\mu}(A)$-module $L$ to be h.d. $1 \sigma$-torsion are:

(i) there exists a 1-dimensional f.g. projective $A$-module chain complex $J: J_{1} \rightarrow$ $J_{0}$ with a homology equivalence

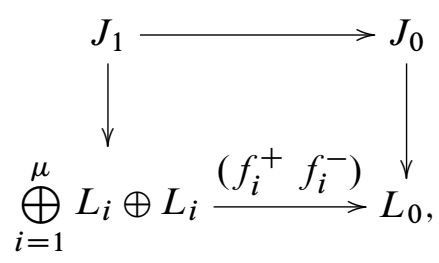

(ii) each $L_{i}(1 \leqslant i \leqslant \mu)$ is an h.d. $1 A$-module,

(iii) the $A\left[F_{\mu}\right]$-module morphism

$$
\left(f_{i}^{+} z_{i}-f_{i}^{-}\right): \bigoplus_{i=1}^{\mu} L_{i}\left[F_{\mu}\right] \rightarrow L_{0}\left[F_{\mu}\right]
$$

is an isomorphism.

If $L$ satisfies these conditions there is defined a commutative diagram of $A$-modules

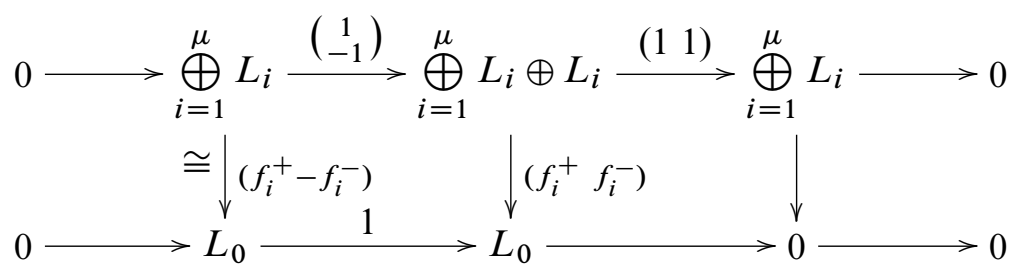

with exact rows and with $\left(f_{i}^{+}-f_{i}^{-}\right)$an isomorphism. There are defined $A$-module isomorphisms

$$
J_{0} \oplus L_{0} \cong J_{0} \oplus \bigoplus_{i=1}^{\mu} L_{i} \cong J_{1},
$$

so that each $L_{i}(0 \leqslant i \leqslant \mu)$ is a f.g. projective $A$-module. 
Example 5.10 The assembly of $A\left[F_{\mu}\right]$-modules is an algebraic analogue of the geometric construction of an $F_{\mu}$-cover $\widetilde{W}$ of a space $W$ from a fundamental domain $U \subset \widetilde{W}$. The subspaces

$$
V_{i}=U \cap z_{i}^{-1} U, \quad z_{i} V_{i}=z_{i} U \cap U \subset U \quad(1 \leqslant i \leqslant \mu)
$$

are disjoint, with embeddings

$$
f_{i}^{+}: V_{i} \rightarrow U ; x \mapsto x, \quad f_{i}^{-}: V_{i} \rightarrow U ; x \mapsto z_{i} x \quad(1 \leqslant i \leqslant \mu),
$$

and $\widetilde{W}$ can be constructed by glueing together $F_{\mu}$ copies of $U$

$$
\begin{aligned}
\widetilde{W} & =\left(F_{\mu} \times U\right) /\left\{\left(g, f_{i}^{+}(x)\right) \sim\left(g z_{i}, f_{i}^{-}(x)\right) \mid g \in F_{\mu}, x \in V_{i}, 1 \leqslant i \leqslant \mu\right\} \\
& =\bigcup_{g \in F_{\mu}} g U \text { with } U \cap z_{i}^{-1} U=V_{i} .
\end{aligned}
$$

Such a situation arises if $W$ is a manifold with a surjection $\pi_{1}(W) \rightarrow F_{\mu}$, eg a boundary link exterior. The surjection is induced by a map

$$
c: W \rightarrow B F_{\mu}=\bigvee_{\mu} S^{1}
$$

which is transverse regular at $\{1,2, \ldots, \mu\} \subset B F_{\mu}$. Cutting $W$ open at the inverse image codimension-1 submanifolds $V_{i}=c^{-1}(\{i\}) \subset W$ there is obtained a fundamental domain $U \subset \widetilde{W}$ for the pullback $\widetilde{W}=c^{*} E F_{\mu}$ to $W$ of the universal cover $E F_{\mu}$ of $B F_{\mu}$. More generally, suppose that $W$ is a finite $C W$ complex with an $F_{\mu}$ cover $\widetilde{W}$, and that $U \subset \widetilde{W}$ is a fundamental domain which is a subcomplex. The embeddings $f_{i}^{+}, f_{i}^{-}: V_{i} \rightarrow U$ induce inclusions of the cellular f.g. free $\mathbb{Z}$-module chain complexes $f_{i}^{+}, f_{i}^{-}: C\left(V_{i}\right) \rightarrow C(U)$. The f.g. projective $T_{\mu}(\mathbb{Z})$-module chain complex $C=\left(C(U), C\left(V_{i}\right), f_{i}^{+}, f_{i}^{-}\right)$has assembly the cellular f.g. free $\mathbb{Z}\left[F_{\mu}\right]-$ module chain complex of $\widetilde{W}$

$$
\operatorname{coker}\left(\left(f_{1}^{+} z_{1}-f_{1}^{-} \ldots f_{\mu}^{+} z_{\mu}-f_{\mu}^{-}\right): \bigoplus_{i=1}^{\mu} C\left(V_{i}\right)\left[F_{\mu}\right] \rightarrow C(U)\left[F_{\mu}\right]\right)=C(\widetilde{W}),
$$

such that

$$
C(\widetilde{W})_{r}=\operatorname{coker}\left(\left(f_{1}^{+} \ldots f_{\mu}^{+}\right): \bigoplus_{i=1}^{\mu} C\left(V_{i}\right)_{r} \rightarrow C(U)_{r}\right)\left[F_{\mu}\right]
$$

Theorem 5.11 The algebraic $K$-groups of $A\left[F_{\mu}\right]$ split as

$$
K_{*}\left(A\left[F_{\mu}\right]\right)=K_{*}(A) \oplus \bigoplus_{\mu} K_{*-1}(A) \oplus \widetilde{\operatorname{Prim}}_{*-1}(A) .
$$


Proof By Proposition 5.9 the universal localization

$$
\mathcal{F}: A\left[F_{\mu}\right] \rightarrow \sigma^{-1} T_{\mu}(A)=M_{\mu+1}(A)
$$

is injective and stably flat. The noncommutative localization exact sequence of Neeman and Ranicki [30; 31]

$$
\begin{aligned}
\cdots \longrightarrow K_{n+1}\left(\sigma^{-1} T_{\mu}(A)\right) & \longrightarrow K_{n}\left(H\left(T_{\mu}(A), \sigma\right)\right) \\
& \longrightarrow K_{n}\left(T_{\mu}(A)\right) \longrightarrow K_{n}\left(\sigma^{-1} T_{\mu}(A)\right) \longrightarrow \cdots
\end{aligned}
$$

is given by

$$
\cdots \rightarrow K_{n+1}\left(A\left[F_{\mu}\right]\right) \rightarrow \operatorname{Prim}_{n}(A) \rightarrow \bigoplus_{\mu+1} K_{n}(A) \rightarrow K_{n}\left(A\left[F_{\mu}\right]\right) \rightarrow \cdots
$$

with $\operatorname{Prim}_{n}(A) \rightarrow K_{n}\left(T_{\mu}(A)\right)=\bigoplus_{\mu+1} K_{n}(A)$ induced by

$$
\operatorname{Prim}(A) \rightarrow \prod_{\mu+1} \operatorname{Proj}(A) ;\left(P, e,\left\{\pi_{i}\right\}\right) \mapsto\left(P, P_{1}, P_{2}, \ldots, P_{\mu}\right)
$$

so that

$$
\begin{aligned}
\operatorname{Prim}_{n}(A) & =\bigoplus_{2 \mu} K_{n}(A) \oplus \underset{\operatorname{Prim}_{n}}{ }(A) \rightarrow \bigoplus_{\mu+1} K_{n}(A) \\
\left(x_{1}^{+}, x_{1}^{-}, x_{2}^{+}, x_{2}^{-}, \ldots, x_{\mu}^{+}, x_{\mu}^{-}, \tilde{x}\right) & \\
& \longmapsto\left(\sum_{i=1}^{\mu}\left(x_{i}^{+}+x_{i}^{-}\right), x_{1}^{+}+x_{1}^{-}, x_{2}^{+}+x_{2}^{-}, \ldots, x_{\mu}^{+}+x_{\mu}^{-}\right) .
\end{aligned}
$$

This completes the proof.

Definition 5.12 Let $\mathcal{G}: A\left[F_{\mu}\right] \rightarrow \Sigma^{-1} A\left[F_{\mu}\right]$ be the universal localization inverting the set $\Sigma$ of morphisms of f.g. projective $A\left[F_{\mu}\right]$-modules which induce an isomorphism of f.g. projective $A$-modules under the augmentation $\epsilon: A\left[F_{\mu}\right] \rightarrow A ; z_{i} \mapsto 1$.

Proposition 5.13 (i) The universal localization $\mathcal{G}: A\left[F_{\mu}\right] \rightarrow \Sigma^{-1} A\left[F_{\mu}\right]$ is injective. The h.d. $1 \Sigma$-torsion $A\left[F_{\mu}\right]$-module category is

$$
H\left(A\left[F_{\mu}\right], \Sigma\right)=\mathcal{B} \operatorname{la}(A) .
$$

(ii) The composite

$$
\mathcal{G F}: T_{\mu}(A) \stackrel{\mathcal{F}}{\longrightarrow} \sigma^{-1} T_{\mu}(A)=M_{\mu+1}\left(A\left[F_{\mu}\right]\right) \stackrel{\mathcal{G}}{\longrightarrow} \tau^{-1} T_{\mu}(A)=M_{\mu+1}\left(\Sigma^{-1} A\left[F_{\mu}\right]\right)
$$


is the universal localization inverting the set $\tau$ of morphisms of f.g. projective $T_{\mu}(A)-$ modules which become isomorphisms under the composite

$$
\epsilon \mathcal{F}: T_{\mu}(A) \stackrel{\mathcal{F}}{\longrightarrow} \sigma^{-1} T_{\mu}(A)=M_{\mu+1}\left(A\left[F_{\mu}\right]\right) \stackrel{\epsilon}{\longrightarrow} M_{\mu+1}(A) .
$$

(iii) A $T_{\mu}(A)$-module $L=\left(L_{i}, f_{i}^{+}, f_{i}^{-}\right)$is h.d. $1 \tau$-torsion if and only if $L_{0}, \ldots, L_{\mu}$ are f.g. projective $A$-modules and the $A$-module morphism

$$
f=\left(f_{1}^{+}-f_{1}^{-} f_{2}^{+}-f_{2}^{-} \ldots f_{\mu}^{+}-f_{\mu}^{-}\right): L_{1} \oplus L_{2} \oplus \cdots \oplus L_{\mu} \rightarrow L_{0}
$$

is an isomorphism, if and only if

$$
\left(P, e,\left\{\pi_{i}\right\}\right)=\left(\bigoplus_{i=1}^{\mu} L_{i}, f^{-1}\left(f_{1}^{+} f_{2}^{+} \ldots f_{\mu}^{+}\right),\left\{\pi_{i}\right\}\right)
$$

is a f.g. projective Seifert $A$-module. The functor

$$
\operatorname{Sei}(A) \rightarrow H\left(T_{\mu}(A), \tau\right) ;\left(P, e,\left\{\pi_{i}\right\}\right) \mapsto\left(P, P_{i}, e \pi_{i},(e-1) \pi_{i}\right)
$$

is an equivalence of exact categories. The assembly of $\left(L_{i}, f_{i}^{+}, f_{i}^{-}\right)$is the covering Blanchfield $A\left[F_{\mu}\right]$-module of $\left(P, e,\left\{\pi_{i}\right\}\right)$

$$
\begin{aligned}
\operatorname{coker}\left(\left(f_{1}^{+} z_{1}-f_{1}^{-} \ldots f_{\mu}^{+} z_{\mu}-f_{\mu}^{-}\right): \bigoplus_{i=1}^{\mu} L_{i}\left[F_{\mu}\right] \rightarrow L_{0}\left[F_{\mu}\right]\right) \\
\quad=\operatorname{coker}\left(1-e+z e: P\left[F_{\mu}\right] \rightarrow P\left[F_{\mu}\right]\right)=B\left(P, e,\left\{\pi_{i}\right\}\right),
\end{aligned}
$$

so that up to equivalence

$$
\mathcal{F}=B: H\left(T_{\mu}(A), \tau\right)=\operatorname{Sei}(A) \rightarrow H\left(M_{\mu+1}\left(A\left[F_{\mu}\right]\right), \tau\right)=\operatorname{Bla}(A) .
$$

(iv) The forgetful functor

$$
\operatorname{Sei}(A) \rightarrow \prod_{\mu} \mathcal{P} \operatorname{roj}(A) ;\left(P, e,\left\{\pi_{i}\right\}\right) \mapsto\left(P_{1}, P_{2}, \ldots, P_{\mu}\right)
$$

is split by

$$
\prod_{\mu} \operatorname{Proj}(A) \rightarrow \mathcal{P} \operatorname{rim}(A) ;\left(P_{1}, P_{2}, \ldots, P_{\mu}\right) \mapsto\left(\bigoplus_{i=1}^{\mu} P_{i}, 0,\left\{\pi_{i}\right\}\right)
$$

The reduced $K$-groups defined by

$$
\widetilde{\operatorname{Sei}}_{*}(A)=\operatorname{ker}\left(\operatorname{Sei}_{*}(A) \rightarrow \bigoplus_{\mu} K_{*}(A)\right)
$$


are such that

$$
K_{*}\left(H\left(T_{\mu}(A), \tau\right)\right)=\operatorname{Sei}_{*}(A)=\bigoplus_{\mu} K_{*}(A) \oplus \widetilde{\operatorname{Sei}}_{*}(A) .
$$

Proof (i) The Magnus-Fox embedding $A\left[F_{\mu}\right] \rightarrow A\left\langle\left\langle x_{1}, \ldots, x_{\mu}\right\rangle\right\rangle$ is $\Sigma$-inverting, so that there is a unique factorization

$$
A\left[F_{\mu}\right] \rightarrow \Sigma^{-1} A\left[F_{\mu}\right] \rightarrow A\left\langle\left\langle x_{1}, x_{2}, \ldots, x_{\mu}\right\rangle\right\rangle .
$$

The identification $H\left(A\left[F_{\mu}\right], \Sigma\right)=\mathcal{B} l a(A)$ is a formality, as is the identification $\mathcal{P} \operatorname{roj}\left(A\left[F_{\mu}\right]\right)=\mathcal{P}\left(\mathcal{P r o j}_{A}\left(A\left[F_{\mu}\right]\right)\right)$ with $\operatorname{Proj}_{A}\left(A\left[F_{\mu}\right]\right) \subseteq \mathcal{P} \operatorname{roj}\left(A\left[F_{\mu}\right]\right)$ the full subcategory with objects isomorphic to the f.g. projective $A\left[F_{\mu}\right]$-modules $P\left[F_{\mu}\right]$ induced from f.g. projective $A$-modules $P$.

(ii)-(iv) By construction, working as in the proof of Proposition 5.9 (iv) to show that if $L=\left(L_{i}, f_{i}^{+}, f_{i}^{-}\right)$is an h.d. $1 \tau$-torsion $T_{\mu}(A)$-module then $L_{0}, L_{1}, \ldots, L_{\mu}$ are f.g. projective $A$-modules.

Theorem 5.14 (i) The algebraic $K$-groups of $\mathcal{P} \operatorname{rim}(A), \mathcal{S} e i(A)$ and $\mathcal{B l a}(A)$ fit into a commutative braid of exact sequences

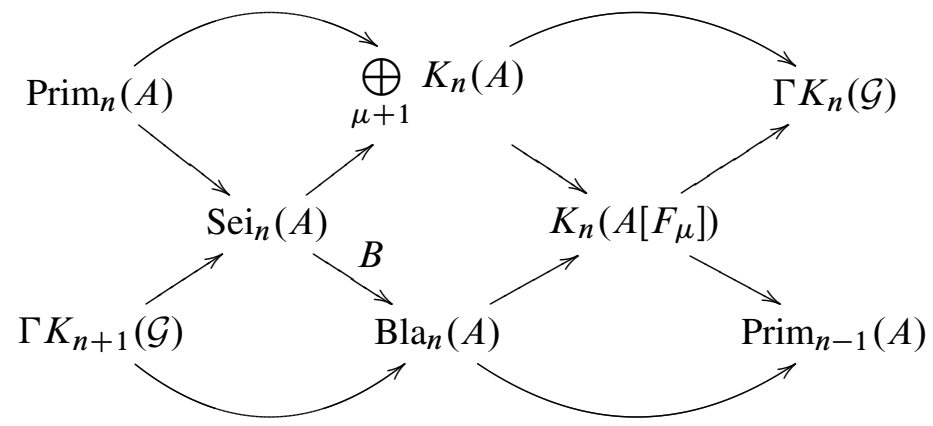

for $n \in \mathbb{Z}$, with $\mathcal{G}: A\left[F_{\mu}\right] \rightarrow \Sigma^{-1} A\left[F_{\mu}\right]$ the universal localization and

$$
\begin{aligned}
K_{*}\left(T_{\mu}(A)\right) & =\bigoplus K_{*}(A), \\
K_{*}\left(H\left(T_{\mu}(A), \sigma\right)\right) & =(\operatorname{Sei}, \mathrm{Bla})_{*}(A)=\operatorname{Prim}_{*}(A)=\bigoplus \bigoplus_{2 \mu} K_{*}(A) \oplus \widetilde{\operatorname{Prim}_{*}}(A), \\
K_{*}\left(H\left(T_{\mu}(A), \tau\right)\right) & =\operatorname{Sei}_{*}(A)=\bigoplus_{\mu} K_{*}(A) \oplus \widetilde{\operatorname{Sei}}_{*}(A), \\
K_{*}\left(H\left(A\left[F_{\mu}\right], \Sigma\right)\right) & =\operatorname{Bla}_{*}(A)=\bigoplus_{\mu} K_{*-1}(A) \oplus \widetilde{\operatorname{Bla}_{*}}(A), \\
\Gamma K_{*}(\mathcal{G}) & =K_{*}(A) \oplus \widetilde{\operatorname{Sei}_{*-1}}(A)\left(=K_{*}\left(\Sigma^{-1} A\left[F_{\mu}\right]\right) \text { for } * \leqslant 1\right)
\end{aligned}
$$


The reduced $K$-groups fit into a long exact sequence

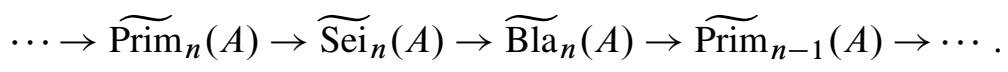

(ii) If $\mathcal{G}: A\left[F_{\mu}\right] \rightarrow \Sigma^{-1} A\left[F_{\mu}\right]$ is stably flat then

$$
\Gamma K_{n}(\mathcal{G})=K_{n}\left(\Sigma^{-1} A\left[F_{\mu}\right]\right)=K_{n}(A) \oplus \widetilde{\operatorname{Sei}}_{n-1}(A)
$$

for all $n \in \mathbb{Z}$.

Proof (i) Consider the commutative square of Waldhausen categories

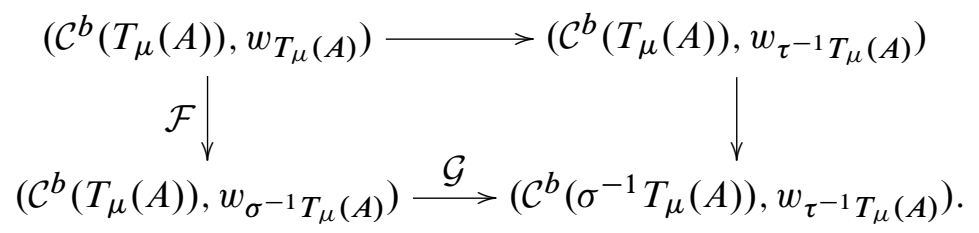

Since $\mathcal{F}: T_{\mu}(A) \rightarrow \sigma^{-1} T_{\mu}(A)=M_{\mu+1}\left(A\left[F_{\mu}\right]\right)$ is stably flat there are defined equivalences

$\left(\mathcal{C}^{b}\left(T_{\mu}(A)\right), w_{\sigma^{-1}} T_{\mu}(A)\right) \approx\left(\mathcal{C}^{b}\left(\sigma^{-1} T_{\mu}(A)\right), w_{\sigma^{-1} T_{\mu}(A)}\right) \approx\left(\mathcal{C}^{b}\left(A\left[F_{\mu}\right]\right), w_{A\left[F_{\mu}\right]}\right)$

which induce homotopy equivalences

$$
K \mathcal{P}\left(\mathcal{C}^{b}\left(T_{\mu}(A)\right), w_{\sigma^{-1}} T_{\mu}(A)\right) \simeq K\left(\sigma^{-1} T_{\mu}(A)\right) \simeq K\left(A\left[F_{\mu}\right]\right) .
$$

Also, since $\tau^{-1} T_{\mu}(A)=M_{\mu+1}\left(\Sigma^{-1} A\left[F_{\mu}\right]\right)$ the functor

$$
\left(\mathcal{C}^{b}\left(T_{\mu}(A)\right), w_{\tau^{-1}} T_{\mu}(A)\right) \rightarrow\left(\mathcal{C}^{b}\left(\sigma^{-1} T_{\mu}(A)\right), w_{\tau^{-1}} T_{\mu}(A)\right)
$$

induces an equivalence of the homotopy categories

$$
D\left(\mathcal{C}^{b}\left(T_{\mu}(A)\right), w_{\tau^{-1}} T_{\mu}(A)\right) \approx\left(\mathcal{C}^{b}\left(\sigma^{-1} T_{\mu}(A), w_{\tau^{-1} T_{\mu}(A)}\right) .\right.
$$

The composite of this equivalence and the Morita equivalence

$$
D\left(\mathcal{C}^{b}\left(\sigma^{-1} T_{\mu}(A)\right), w_{\tau^{-1}} T_{\mu}(A)\right) \approx D\left(\mathcal{C}^{b}\left(A\left[F_{\mu}\right]\right), w_{\Sigma^{-1} A\left[F_{\mu}\right]}\right)
$$

induces a homotopy equivalence

$$
\begin{aligned}
K \mathcal{P}\left(\mathcal{C}^{b}\left(\sigma^{-1} T_{\mu}(A)\right), w_{\tau^{-1} T_{\mu}(A)}\right) \simeq & K \mathcal{P}\left(\mathcal{C}^{b}\left(A\left[F_{\mu}\right]\right), w_{\Sigma^{-1} A\left[F_{\mu}\right]}\right) \\
& =\Gamma K\left(\mathcal{G}: A\left[F_{\mu}\right] \rightarrow \Sigma^{-1} A\left[F_{\mu}\right]\right) .
\end{aligned}
$$


Thus Propositions 5.6, 5.9 and 5.13 give a braid of Waldhausen categories

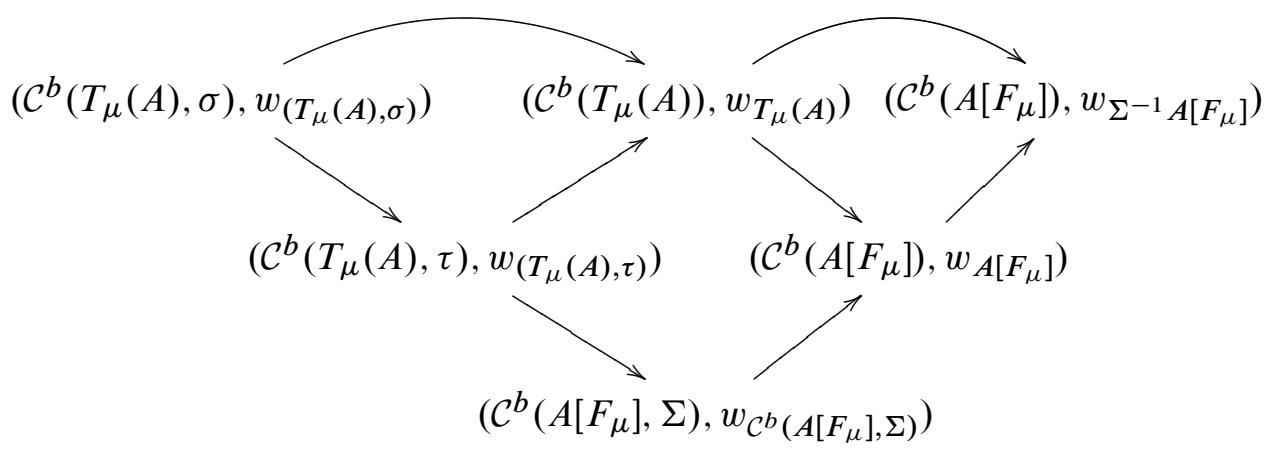

inducing a commutative braid of exact sequences

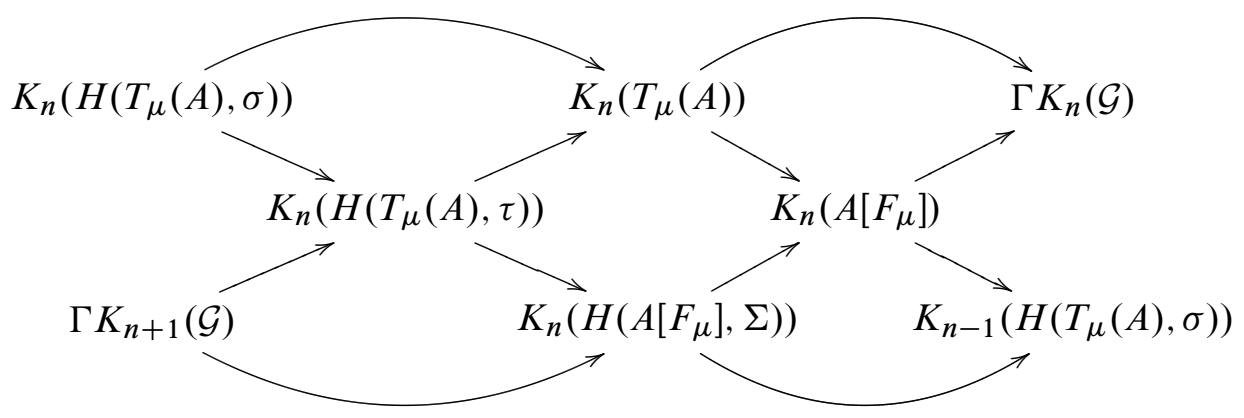

Split off the reduced $K$-groups in

$$
\begin{aligned}
\operatorname{Prim}_{*}(A) & =\bigoplus_{2 \mu} K_{*}(A) \oplus \widetilde{\operatorname{Prim}_{*}}(A) \\
\operatorname{Sei}_{*}(A) & =\bigoplus_{\mu} K_{*}(A) \oplus \widetilde{\operatorname{Sei}_{*}}(A)
\end{aligned}
$$

from the long exact sequence

$$
\cdots \rightarrow \operatorname{Prim}_{n}(A) \rightarrow \operatorname{Sei}_{n}(A) \rightarrow \operatorname{Bla}_{n}(A) \rightarrow \operatorname{Prim}_{n-1}(A) \rightarrow \cdots
$$

to define the reduced $K$-groups in

$$
\mathrm{Bla}_{*}(A)=\bigoplus_{\mu} K_{*-1}(A) \oplus \widetilde{\mathrm{Bla}_{*}}(A)
$$

and to obtain the long exact sequence

$$
\cdots \rightarrow \widetilde{\operatorname{Prim}}_{n}(A) \rightarrow{\widetilde{\operatorname{Sei}_{n}}}_{n}(A) \rightarrow{\widetilde{\operatorname{Bla}_{n}}}_{n}(A) \rightarrow \widetilde{\operatorname{Prim}}_{n-1}(A) \rightarrow \cdots
$$

(ii) This is a special case of Proposition 5.6 (ii). 
This completes the proofs of Theorems D and E of the Introduction.

Remark 5.15 Unfortunately, we do not know if the universal localization $\Sigma^{-1} A\left[F_{\mu}\right]$ is stably flat in general. See Dicks and Sontag [11], Farber and Vogel [16] for proofs that $\Sigma^{-1} A\left[F_{\mu}\right]$ is stably flat when $A$ is a principal ideal domain, and Ara and Dicks [1, Theorem 4.4] when $A$ is a von Neumann regular ring or a commutative Bezout domain.

Remark 5.16 Sheiham [40] computed

$$
K_{1}\left(\Sigma^{-1} A\left[F_{\mu}\right]\right)=K_{1}(A) \oplus \epsilon_{\Sigma}^{-1}(1) / C
$$

with $\epsilon_{\Sigma}: \Sigma^{-1} A\left[F_{\mu}\right] \rightarrow A$ the factorization of the augmentation map $\epsilon: A\left[F_{\mu}\right] \rightarrow A$ and $C \subseteq \epsilon_{\Sigma}^{-1}(1)$ the subgroup generated by the commutators

$$
(1+a b)(1+b a)^{-1} \quad\left(a, b \in \Sigma^{-1} A\left[F_{\mu}\right], \epsilon(a b)=\epsilon(b a)=0\right) .
$$

It follows from the splitting given by Theorem 5.14 (i)

$$
K_{1}\left(\Sigma^{-1} A\left[F_{\mu}\right]\right)=K_{1}(A) \oplus{\widetilde{\operatorname{Sei}_{0}}}_{0}(A)
$$

that there is defined an isomorphism

$$
\widetilde{\operatorname{Sei}}_{0}(A) \stackrel{\cong}{\longrightarrow} \epsilon_{\Sigma}^{-1}(1) / C ;\left(P, e,\left\{\pi_{i}\right\}\right) \mapsto D\left(1-e+e z: P\left[F_{\mu}\right] \rightarrow P\left[F_{\mu}\right]\right)
$$

with $D$ the generalized Dieudonné noncommutative determinant of [40, 4.3].

Example 5.17 (i) The algebraic $K$-groups of $\mathbb{Z}\left[F_{\mu}\right]$ are such that

$$
\begin{aligned}
& K_{*}\left(\mathbb{Z}\left[F_{\mu}\right]\right)=K_{*}(\mathbb{Z}) \oplus \bigoplus_{\mu} K_{*-1}(\mathbb{Z}), \\
& K_{n}\left(\mathbb{Z}\left[F_{\mu}\right]\right)=K_{n}(\mathbb{Z})= \begin{cases}\mathbb{Z} & \text { if } n=0 \\
0 & \text { if } n \leqslant-1\end{cases}
\end{aligned}
$$

by Stallings [44], Gersten [19], Bass [4, XII] and Waldhausen [49; 48], so that

$$
\begin{aligned}
& \mathcal{F} l k(\mathbb{Z})=\mathcal{B l a}(\mathbb{Z}), \quad \widetilde{\operatorname{Prim}_{*}}(\mathbb{Z})=0, \\
& K_{*+1}\left(\Sigma^{-1} \mathbb{Z}\left[F_{\mu}\right]\right) / K_{*+1}(\mathbb{Z})={\widetilde{\operatorname{Sei}_{*}}}_{*}(\mathbb{Z})=\operatorname{Flk}_{*}(\mathbb{Z})=\mathrm{Bla}_{*}(\mathbb{Z}), \\
& K_{*}\left(H\left(\mathbb{Z}\left[F_{\mu}\right], \Sigma\right)\right)=\bigoplus_{\mu} K_{*-1}(\mathbb{Z}) \oplus \widetilde{\operatorname{Sei}}_{*}(\mathbb{Z}), \\
& K_{n}\left(H\left(\mathbb{Z}\left[F_{\mu}\right], \Sigma\right)\right)=\widetilde{\operatorname{Sei}_{n}}(\mathbb{Z}) \quad(n \leqslant 0) .
\end{aligned}
$$

(ii) Given a $\mu$-component boundary link $\ell: \bigsqcup_{\mu} S^{n} \subset S^{n+2}$ with exterior $W$ and given a $\mu$-component Seifert surface $V=V_{1} \sqcup V_{2} \sqcup \ldots \sqcup V_{\mu} \subset S^{n+2}$ for $\ell$ let 
$\dot{C}(W),\left(\dot{C}(V), e,\left\{\pi_{i}\right\}\right)$ be the chain complexes defined in Example 3.13. Thus $\dot{C}(\widetilde{W})$ is a $\Sigma^{-1} \mathbb{Z}\left[F_{\mu}\right]$-acyclic $(n+2)$-dimensional f.g. free $\mathbb{Z}\left[F_{\mu}\right]$-module chain complex, $\left(\dot{C}(V), e,\left\{\pi_{i}\right\}\right)$ is an $(n+1)$-dimensional chain complex in $\mathcal{S e i}(\mathbb{Z})$, and $B\left(\dot{C}(V), e,\left\{\pi_{i}\right\}\right)$ is an $(n+1)$-dimensional chain complex in $\mathcal{F} l k(\mathbb{Z})$ with a homology equivalence $\dot{C}(\widetilde{W}) \rightarrow B\left(\dot{C}(V), e,\left\{\pi_{i}\right\}\right)$. The torsion

$$
\begin{aligned}
\tau(\ell) & =\tau\left(\Sigma^{-1} \dot{C}(\widetilde{W})\right) \\
= & \left(\dot{C}(V), e,\left\{\pi_{i}\right\}\right)=\sum_{r=0}^{n+1}(-)^{r}\left(\dot{C}_{r}(V), e,\left\{\pi_{i}\right\}\right)=[\dot{C}(\widetilde{W})] \\
& \quad \in K_{1}\left(\Sigma^{-1} \mathbb{Z}\left[F_{\mu}\right]\right) / K_{1}(\mathbb{Z})=K_{0}\left(H\left(\mathbb{Z}\left[F_{\mu}\right], \Sigma\right)\right)=\widetilde{\operatorname{Sei}}_{0}(\mathbb{Z})=\mathrm{Bla}_{0}(\mathbb{Z})
\end{aligned}
$$

is an isotopy invariant of $\ell$, given by Sheiham [40] to be the generalized Dieudonné determinant

$$
\tau(\ell)=\sum_{r=0}^{n+1}(-)^{r} D\left(1-e+e z: \dot{C}_{r}(V)\left[F_{\mu}\right] \rightarrow \dot{C}_{r}(V)\left[F_{\mu}\right]\right) \in \widetilde{\operatorname{Sei}_{0}}(\mathbb{Z})=\epsilon_{\Sigma}^{-1}(1) / C
$$

with $\epsilon_{\Sigma}: \Sigma^{-1} \mathbb{Z}\left[F_{\mu}\right] \rightarrow \mathbb{Z}$ and $C \subseteq \epsilon_{\Sigma}^{-1}(1)$ as recalled in Remark 5.16. The $\mathbb{Z}\left[F_{\mu}\right]-$ modules $\dot{H}_{r}(\widetilde{W}) / \mathbb{Z}$-torsion $(0 \leqslant r \leqslant n+1)$ are h.d. $1 F_{\mu}$-link modules, and

$$
\begin{aligned}
\tau(\ell) & =\sum_{r=0}^{n+1}(-)^{r} D\left(1-e+e z: \dot{H}_{r}(V)\left[F_{\mu}\right] \rightarrow \dot{H}_{r}(V)\left[F_{\mu}\right]\right) \\
= & \sum_{r=0}^{n+1}(-)^{r}\left[\dot{H}_{r}(\widetilde{W}) / \mathbb{Z} \text {-torsion }\right] \\
\in & K_{1}\left(\Sigma^{-1} \mathbb{Z}\left[F_{\mu}\right]\right) / K_{1}(\mathbb{Z})=K_{0}\left(H\left(\mathbb{Z}\left[F_{\mu}\right], \Sigma\right)\right) \\
& =\widetilde{\operatorname{Sei}_{0}}(\mathbb{Z})=\mathrm{Bla}_{0}(\mathbb{Z})=\left(\Sigma^{-1} \mathbb{Z}\left[F_{\mu}\right]\right)^{\bullet} /\{ \pm 1\} .
\end{aligned}
$$

For $\mu=1$ this is just the Reidemeister torsion of a knot $\ell: S^{n} \subset S^{n+2}$, which is the alternating product of the Alexander polynomials

$$
\begin{aligned}
& \tau(\ell)= \sum_{r=0}^{n+1}(-)^{r} \operatorname{det}\left(1-e+e z: \dot{H}_{r}(V)\left[z, z^{-1}\right] \rightarrow \dot{H}_{r}(V)\left[z, z^{-1}\right]\right) \\
&=\sum_{r=0}^{n+1}(-)^{r}\left[\dot{H}_{r}(\widetilde{W}) / \mathbb{Z} \text {-torsion }\right] \\
& \quad \in K_{1}\left(\Sigma^{-1} \mathbb{Z}\left[z, z^{-1}\right]\right) / K_{1}(\mathbb{Z})=K_{0}\left(H\left(\mathbb{Z}\left[z, z^{-1}\right], \Sigma\right)\right) \\
& \quad=\widetilde{\operatorname{Sei}}_{0}(\mathbb{Z})=\operatorname{Bla}_{0}(\mathbb{Z})=\widetilde{\operatorname{End}}_{0}(\mathbb{Z})=\left(\Sigma^{-1} \mathbb{Z}\left[z, z^{-1}\right]\right)^{\bullet} /\{ \pm 1\}
\end{aligned}
$$

(Milnor [29], cf [33, Example 17.11]). 
(iii) The isotopy classes of simple $\mu$-component boundary links $\ell$ : $\bigsqcup_{\mu} S^{2 q-1} \subset$ $S^{2 q+1}$ for $q \geqslant 3$ are in one-one correspondence with the ' $l$-equivalence classes of Seifert matrices' (Liang [27], generalizing the case $\mu=1$ due to Levine [25]), and also with the ' $R$-equivalence classes of $(-)^{q}$-symmetric isometry structures of multiplicity $\mu^{\prime}$ (Farber [15, 4.7]). For simple $\ell H_{q}(\widetilde{W})$ is an h.d. $1 F_{\mu}$-link module, and the torsion

$$
\tau(\ell)=(-)^{q}\left[H_{q}(\widetilde{W})\right] \in \widetilde{\operatorname{Sei}_{0}}(\mathbb{Z})=\operatorname{Flk}_{0}(\mathbb{Z})=\operatorname{Bla}_{0}(\mathbb{Z})
$$

is just the $K$-theory part of these complete isotopy invariants for $q \geqslant 3$.

\section{References}

[1] P Ara, W Dicks, Universal localizations embedded in power-series rings, Forum Mathematicum (to appear)

[2] P Balmer, M Schlichting, Idempotent completion of triangulated categories, J. Algebra 236 (2001) 819-834 MR1813503

[3] H Bass, Projective modules over free groups are free, J. Algebra 1 (1964) 367-373 MR0178032

[4] H Bass, Algebraic K-theory, W A Benjamin (1968) MR0249491 Downloadable from: http://www.math.uni-bielefeld.de/ rehmann/DML/

[5] H Bass, A Heller, R G Swan, The Whitehead group of a polynomial extension, Inst. Hautes Études Sci. Publ. Math. (1964) 61-79 MR0174605

[6] A J Berrick, M E Keating, The $K$-theory of triangular matrix rings, from: "Applications of algebraic $K$-theory to algebraic geometry and number theory, Part I, II (Boulder, Colo., 1983)”, Contemp. Math. 55, Amer. Math. Soc., Providence, RI (1986) 69-74 MR862629

[7] F Borceux, Handbook of categorical algebra. 1, Encyclopedia of Mathematics and its Applications 50, Cambridge University Press, Cambridge (1994) MR1291599

[8] S E Cappell, J L Shaneson, The codimension two placement problem and homology equivalent manifolds, Ann. of Math. (2) 99 (1974) 277-348 MR0339216

[9] S E Cappell, J L Shaneson, Link cobordism, Comment. Math. Helv. 55 (1980) 20-49 MR569244

[10] P M Cohn, Free rings and their relations, Academic Press (1971) MR0371938

[11] W Dicks, E D Sontag, Sylvester domains, J. Pure Appl. Algebra 13 (1978) 243-275 MR509164

[12] J Duval, Forme de Blanchfield et cobordisme d'entrelacs bords, Comment. Math. Helv. 61 (1986) 617-635 MR870709 
[13] M Farber, Hermitian forms on link modules, Comment. Math. Helv. 66 (1991) 189-236 MR1107839

[14] M Farber, Noncommutative rational functions and boundary links, Math. Ann. 293 (1992) 543-568 MR1170526

[15] M Farber, Stable-homotopy and homology invariants of boundary links, Trans. Amer. Math. Soc. 334 (1992) 455-477 MR1079054

[16] M Farber, P Vogel, The Cohn localization of the free group ring, Math. Proc. Cambridge Philos. Soc. 111 (1992) 433-443 MR1151322

[17] P Gabriel, Des catégories abéliennes, Bull. Soc. Math. France 90 (1962) 323-448 MR0232821

[18] P Gabriel, M Zisman, Calculus of fractions and homotopy theory, Ergebnisse series 35, Springer, New York (1967) MR0210125

[19] S M Gersten, On class groups of free products, Ann. of Math. (2) 87 (1968) 392-398 MR0224655

[20] H Gillet, Riemann-Roch theorems for higher algebraic K-theory, Adv. in Math. 40 (1981) 203-289 MR624666

[21] D R Grayson, Exact sequences in algebraic K-theory, Illinois J. Math. 31 (1987) 598-617 MR909785

[22] M A Gutiérrez, Boundary links and an unlinking theorem, Trans. Amer. Math. Soc. 171 (1972) 491-499 MR0310902

[23] A Haghany, K Varadarajan, Study of modules over formal triangular matrix rings, J. Pure Appl. Algebra 147 (2000) 41-58 MR1744654

[24] B Keller, Derived categories and their uses, from: "Handbook of algebra, Vol. 1", North-Holland, Amsterdam (1996) 671-701 MR1421815

[25] J Levine, An algebraic classification of some knots of codimension two, Comment. Math. Helv. 45 (1970) 185-198 MR0266226

[26] J Levine, Knot modules I, Trans. Amer. Math. Soc. 229 (1977) 1-50 MR0461518

[27] C C Liang, An algebraic classification of some links of codimension two, Proc. Amer. Math. Soc. 67 (1977) 147-151 MR0458439

[28] W Lück, A Ranicki, Chain homotopy projections, J. Algebra 120 (1989) 361-391 MR989903

[29] J W Milnor, Infinite cyclic coverings, from: "Conference on the Topology of Manifolds (Michigan State Univ., E. Lansing, 1967)”, Prindle, Weber \& Schmidt, Boston (1968) 115-133 MR0242163

[30] A Neeman, A Ranicki, Noncommutative localisation and chain complexes I: Algebraic $K$ - and L-theory arXiv:math.RA/0109118 
[31] A Neeman, A Ranicki, Noncommutative localisation in algebraic $K$-theory I, Geom. Topol. 8 (2004) 1385-1425 MR2119300

[32] D Quillen, Higher algebraic $K$-theory $I$, from: "Algebraic $K$-theory I: Higher $K-$ theories (Proc. Conf., Battelle Memorial Inst., Seattle, Wash., 1972)", Springer, Berlin (1973) 85-147. Lecture Notes in Math., Vol. 341 MR0338129

[33] A Ranicki, High-dimensional knot theory, Springer Monographs in Mathematics, Springer, New York (1998) MR1713074

[34] A Ranicki, Blanchfield and Seifert algebra in high-dimensional knot theory, Mosc. Math. J. 3 (2003) 1333-1367 MR2058802

[35] A Ranicki, Algebraic and combinatorial codimension-1 transversality, from: "Proceedings of the Casson Fest", (C Gordon, R Y, editors), Geom. Topol. Monogr. 7 (2004) 145-180 MR2172482

[36] A Ranicki, Noncommutative localization in topology, from: "Non-commutative localization in algebra and topology", London Math. Soc. Lecture Note Ser. 330, Cambridge Univ. Press, Cambridge (2006) 81-102 MR2222483

[37] N Sato, Free coverings and modules of boundary links, Trans. Amer. Math. Soc. 264 (1981) 499-505 MR603777

[38] M Schlichting, Negative K-theory of derived categories, Math. Z. 253 (2006) 97-134 MR2206639

[39] A H Schofield, Representation of rings over skew fields, London Mathematical Society Lecture Note Series 92, Cambridge University Press, Cambridge (1985) MR800853

[40] D Sheiham, Whitehead groups of localizations and the endomorphism class group, J. Algebra 270 (2003) 261-280 MR2016661

[41] D Sheiham, Invariants of boundary link cobordism II: The Blanchfield-Duval form, from: "Non-commutative localization in algebra and topology (Edinburgh 2002)", LMS Lecture Note Ser. 330, Cambridge Univ. Press, Cambridge (2006) 143-219 MR2222485

[42] D Sheiham, Universal localization of triangular matrix rings, Proc. Amer. Math. Soc 134 (2006) 3465-3474

[43] N Smythe, Boundary links, from: "Wisconsin Topology Seminar", Ann. of Maths. Studies 60, Princeton UP (1966) 69-72

[44] J Stallings, Whitehead torsion of free products, Ann. of Math. (2) 82 (1965) 354-363 MR0179270

[45] R W Thomason, T Trobaugh, Higher algebraic $K$-theory of schemes and of derived categories, from: "The Grothendieck Festschrift, Vol. III", Progr. Math. 88, Birkhäuser, Boston, MA (1990) 247-435 MR1106918 
[46] HF Trotter, Knot modules and Seifert matrices, from: "Knot theory (Proc. Sem., Plans-sur-Bex, 1977)”, Lecture Notes in Math. 685, Springer, Berlin (1978) 291-299 MR521739

[47] F Waldhausen, Whitehead groups of generalized free products, from: "Algebraic $K$-theory II: "Classical" algebraic $K$-theory and connections with arithmetic (Proc. Conf., Battelle Memorial Inst., Seattle, Wash., 1972)”, Springer, Berlin (1973) 155-179. Lecture Notes in Math., Vol. 342 MR0370576

[48] F Waldhausen, Algebraic K-theory of generalized free products, Part 1, Ann. of Math. (2) 108 (1978) 135-204 MR0498807

[49] F Waldhausen, Algebraic $K$-theory of generalized free products, Part 2, Ann. of Math. (2) 108 (1978) 205-256 MR0498808

[50] F Waldhausen, Algebraic $K$-theory of spaces, from: "Algebraic and geometric topology (New Brunswick, N.J., 1983)", Lecture Notes in Math. 1126, Springer, Berlin (1985) 318-419 MR802796

School of Mathematics, University of Edinburgh

Edinburgh EH9 3JZ, Scotland, UK

a.ranicki@ed.ac.uk

Proposed: Wolfgang Lück

Received: 6 October 2005

Seconded: Peter Teichner, Steve Ferry

Revised: 14 July 2006 